\title{
Rényi Resolvability and Its Applications to the Wiretap Channel
}

\author{
Lei Yu and Vincent Y. F. Tan, Senior Member, IEEE
}

\begin{abstract}
The conventional channel resolvability problem refers to the determination of the minimum rate required for an input process so that the output distribution approximates a target distribution in either the total variation distance or the relative entropy. In contrast to previous works, in this paper, we use the (normalized or unnormalized) Rényi divergence (with the Rényi parameter in $[0,2] \cup\{\infty\}$ ) to measure the level of approximation. We also provide asymptotic expressions for normalized Rényi divergence when the Rényi parameter is larger than or equal to 1 as well as (lower and upper) bounds for the case when the same parameter is smaller than 1 . We characterize the Rényi resolvability, which is defined as the minimum rate required to ensure that the Rényi divergence vanishes asymptotically. The Rényi resolvabilities are the same for both the normalized and unnormalized divergence cases. In addition, when the Rényi parameter smaller than 1 , consistent with the traditional case where the Rényi parameter is equal to 1 , the Rényi resolvability equals the minimum mutual information over all input distributions that induce the target output distribution. When the Rényi parameter is larger than 1 the Rényi resolvability is, in general, larger than the mutual information. The optimal Rényi divergence is proven to vanish at least exponentially fast for both of these two cases, as long as the code rate is larger than the Rényi resolvability. The optimal exponential rate of decay for i.i.d. random codes is also characterized exactly. We apply these results to the wiretap channel, and completely characterize the optimal tradeoff between the rates of the secret and non-secret messages when the leakage measure is given by the (unnormalized) Rényi divergence. This tradeoff differs from the conventional setting when the leakage is measured by the traditional mutual information.
\end{abstract}

Index Terms-Channel resolvability, Rényi divergence, Exponent, Soft covering, Wiretap channel, Effective secrecy, Stealthy communication

\section{INTRODUCTION}

How much information is required to simulate a random process through a given channel so that it mimics a target output distribution? This is the so-called channel resolvability problem, studied by Han and Verdú [2]. In [2], the total variation (TV) distance and the normalized relative entropy

This work was supported by a Singapore National Research Foundation (NRF) National Cybersecurity R\&D Grant (R-263-000-C74-281 and NRF2015NCR-NCR003-006). The first author was also supported by a National Natural Science Foundation of China (NSFC) under Grant (61631017). This paper was presented in part at the 2017 International Conference on Information Theoretic Security (ICITS) [1].

L. $\mathrm{Yu}$ is with the Department of Electrical and Computer Engineering, National University of Singapore (NUS), Singapore 117583 (e-mail: leiyu@nus.edu.sg). V. Y. F. Tan is with the Department of Electrical and Computer Engineering and the Department of Mathematics, NUS, Singapore 119076 (e-mail: vtan@nus.edu.sg).

Communicated by M. Bloch, Associate Editor for Shannon Theory.

Copyright (c) 2018 IEEE. Personal use of this material is permitted. However, permission to use this material for any other purposes must be obtained from the IEEE by sending a request to pubs-permissions@ieee.org.
(Kullback-Leibler divergence) were used to measure the level of approximation. The resolvability problem with the unnormalized relative entropy was studied by Hayashi [3], [4]. In [2]-[4] it was shown that in the memoryless case the minimum rates of randomness needed for simulating a channel output under the TV, normalized relative entropy, or unnormalized relative entropy measures are the same, and are all equal to the minimum mutual information over all input distributions that induce the target output distribution. Recently, Liu, Cuff, and Verdú [5] extended the theory of resolvability by using $E_{\gamma}$ metric with $\gamma \geq 1$ to measure the level of approximation. The $E_{\gamma}$ metric reduces to the TV distance when $\gamma=1$, but it is weaker than the TV distance when $\gamma>1$. Hence, the $E_{\gamma}$ metric generalizes the TV distance by weakening the measure. In contrast, we generalize the channel resolvability problem by strengthening the relative entropy measure and considering a continuum of secrecy measures indexed by the Rényi parameter. Furthermore, random variable simulation problems under Rényi divergence measures of all orders in $[0, \infty]$, including the source resolvability problem (the resolvability problem with the identity channel), were studied by the present authors recently in [6]. The exact channel resolvability problem was studied by the present authors in [7], in which the output distribution is required to be exactly equal to the target distribution, and meanwhile, the input process is allowed to be an "asymptotic function" (i.e., not restricted to be a function) of a uniform random variable (or the input process is allowed to be compressed by variable-length codes, not restricted to fixed-length codes).

While the term "channel resolvability" was coined by Han and Verdú in [2], the problem of approximating a given product measure was first studied by Wyner [8]. In [8] Wyner investigated the minimum rate of common randomness to simulate two correlated sources in a distributed fashion such that the distance (e.g., TV distance or relative entropy) between the code-induced distribution and the target source distribution vanishes asymptotically; this rate was coined the common information rate between the two sources. For the achievability part, both channel resolvability and common information problems rely on the so-called soft-covering lemmas [9]. The channel resolvability and common information problems have several interesting applications-including secrecy, channel synthesis, and source coding. For example, in [10] it was used to study the performance of a wiretap channel system under different secrecy measures. In [11] it was used to study the reliability and secrecy exponents of a wiretap channel with cost constraints. In [12] it was used to study the exact secrecy exponents of random code ensembles for the wiretap channel. In [13], Hou and Kramer used ideas from the channel 
resolvability problem to study the effective secrecy capacity (the stealth-secrecy capacity) of wiretap channels. This work is contrasted to the present work in greater detail in Section III. Furthermore, the perfectly stealthy (or covert) communication problem, in which the distribution of the signal overheard by the eavesdropper is required to be exactly equal to the target distribution, was studied by the present authors in [7]. The exact common information problem was studied in [14]-[16], in which the code-induced distribution is required to be exactly equal to the target source distribution, and meanwhile, the common randomness is allowed to be compressed by variablelength codes, not restricted to fixed-length codes.

In contrast to the aforementioned works, we use the (normalized or unnormalized) Rényi divergence to measure the level of approximation between the simulated and target output distributions. As expounded by Iwamoto and Shikata [17], we can quantify equivocation using Rényi measures, thus obtaining a continuum of fundamental limits of information leakage under the effect of various hash functions. These fundamental limits are indexed by the Rényi parameter. Our work is also partly motivated by Shikata [18] who quantified lengths of secret keys in terms of Rényi entropies of general orders and Bai et al. [19] who showed that the Rényi divergence is particularly suited for simplifying some security proofs. Furthermore, it is worth noting that it is quite natural to use various divergences to measure the discrepancy between two distributions. Wyner [8] and Yu and Tan [16], [20], [21] respectively used the KL divergence and the Rényi divergence to measure the level of approximation in the distributed source synthesis problem; Hayashi [3], [4] used the KL divergence to study the channel resolvability problem, and showed the optimal decay exponents of the KL divergence and the total variation are upper bounded by an expression involving the Rényi divergence. In probability theory, Barron [22] and Bobkov, Chistyakov and Götze [23] respectively used the KL divergence and the Rényi divergence to study the central limit theorem, i.e., they used them to measure the discrepancy between the induced distribution of sum of i.i.d. random variables and the normal distribution with the same mean and variance. Furthermore, special instances of Rényi entropies and divergences-including the KL divergence, the Rényi divergence, the collision entropy (the Rényi entropy of order 2), and min-entropy (the Rényi entropy of order $\infty$ )—were used to study various information-theoretic problems (including security, cryptography, and quantum information) in several works in the recent literature [10], [13], [24]-[29]; and these give some operational meanings of the Rényi divergence. For example, in [28], the normalized Rényi entropy of order 2 was used to express an achievable rate for the secret communication over the wiretap channel with non-uniform sources. In [29], the Rényi divergence was used to express an achievable exponent for secure multiplex coding with the leakage measured by mutual information.

\section{A. Main Contributions}

Our main contributions are as follows:

1) We provide finite length and asymptotic expressions for the Rényi divergence between the simulated and target output distributions. We distinguish between the case when the Rényi parameter is at least 1 -in which case we have a tight expression - and the case when the same parameter is smaller than 1 -in which case we only have bounds (which are tight in some regime).

2) We characterize the Rényi resolvability, which is defined as the minimum rate needed to guarantee that the (normalized or unnormalized) Rényi divergence vanishes asymptotically. Interestingly, these two Rényi resolvabilities are the same regardless of whether we employ the normalized or unnormalized Rényi divergences. The Rényi resolvability when the Rényi parameter is at most 1 is just equal to the minimum mutual information over all input distribution that induce target output distribution. This is similar to the traditional case [2][4]. In contrast if the Rényi parameter is greater than 1 , the Rényi resolvability is, in general, larger than the minimum mutual information.

3) We prove that the optimal Rényi divergence between the simulated and target output distributions vanishes (at least) exponentially fast as long as the code rate is larger than the Rényi resolvability (cf. previous point). We also exactly characterize the optimal (ensemble tight) exponential decay rate for the ensemble of i.i.d. random codes. These results are generalizations of the work by Parizi, Telatar and Merhav [12] in which the optimal exponent (leading to an ensemble tight secrecy exponent for the wiretap channel) for the relative entropy was studied. See Remark 22 for further comparisons and contrasts to [12].

4) As a concrete application of the above mathematical results, we consider the wiretap channel and completely characterize the optimal tradeoff between the rates of the secret and non-secret messages when the leakage is measured by the unnormalized Rényi divergence. Note that different from Csiszár and Körner's work (with secrecy measured by the mutual information) [30], the optimal rates tradeoff provided by us are achieved by a single-layered code. Hence, it has a different expression from the one given in [30]. See Remark 26 for a detailed discussion.

It is also worth noting that our work is partly motivated by the work of Hayashi and Tan [26], [27]. In their work, the Rényi divergence was used to measure the level of approximation of a distribution induced by a hash function, typically used for source compression; in our work, it is used to measure the level of approximation of an input process that is sent through a channel. Hence our work can be considered as a counterpart of theirs, just as the channel coding is a counterpart of the source hashing.

\section{B. Notation}

In this paper, we use $P_{X}(x)$ to denote the probability distribution of a random variable $X$, which is also shortly denoted as $P(x)$ (when the random variable $X$ is clear from the context). We also use $P_{X}, \widetilde{P}_{X}$, and $Q_{X}$ to denote various probability distributions with alphabet $\mathcal{X}$. All alphabets considered in 
the sequel are finite. The set of probability distributions on $\mathcal{X}$ is denoted as $\mathcal{P}(\mathcal{X})$, and the set of conditional probability distributions on $\mathcal{Y}$ given a variable in $\mathcal{X}$ is denoted as $\mathcal{P}(\mathcal{Y} \mid \mathcal{X}):=$ $\left\{P_{Y \mid X}: P_{Y \mid X}(\cdot \mid x) \in \mathcal{P}(\mathcal{Y}), x \in \mathcal{X}\right\}$. Given $P_{X}$ and $P_{Y \mid X}$, we write $\left[P_{Y \mid X} \circ P_{X}\right](y):=\sum_{x} P_{Y \mid X}(y \mid x) P_{X}(x)$.

We use $T_{x^{n}}(x):=\frac{1}{n} \sum_{i=1}^{n} 1\left\{x_{i}=x\right\}$ to denote the type (empirical distribution) of a sequence $x^{n}, T_{X}$ and $V_{Y \mid X}$ to respectively denote a type of sequences in $\mathcal{X}^{n}$ and a conditional type of sequences in $\mathcal{Y}^{n}$ (given a sequence $x^{n} \in \mathcal{X}^{n}$ ). For a type $T_{X}$, the type class (set of sequences having the same type $T_{X}$ ) is denoted by $\mathcal{T}_{T_{X}}$. For a conditional type $V_{Y \mid X}$ and a sequence $x^{n}$, the $V$-shell of $x^{n}$ (the set of $y^{n}$ sequences having the same conditional type $V_{Y \mid X}$ given $x^{n}$ ) is denoted by $\mathcal{T}_{V_{Y \mid X}}\left(x^{n}\right)$. The set of types of sequences in $\mathcal{X}^{n}$ is denoted as $\mathcal{P}^{(n)}(\mathcal{X}):=\left\{T_{x^{n}}: x^{n} \in \mathcal{X}^{n}\right\}$. The set of conditional types of sequences in $\mathcal{Y}^{n}$ given a sequence in $\mathcal{X}^{n}$ with the type $T_{X}$ is denoted as $\mathcal{P}^{(n)}\left(\mathcal{Y} \mid T_{X}\right):=\left\{V_{Y \mid X} \in \mathcal{P}(\mathcal{Y} \mid \mathcal{X}): V_{Y \mid X} \times T_{X} \in\right.$ $\left.\mathcal{P}^{(n)}(\mathcal{X} \times \mathcal{Y})\right\}$. For brevity, sometimes we use $T(x, y)$ to denote the joint distributions $T(x) V(y \mid x)$ or $T(y) V(x \mid y)$.

The $\epsilon$-typical set relative to $Q_{X}$ is denoted as $\mathcal{T}_{\epsilon}^{n}\left(Q_{X}\right):=$ $\left\{x^{n} \in \mathcal{X}^{n}:\left|T_{x^{n}}(x)-Q_{X}(x)\right| \leq \epsilon Q_{X}(x), \forall x \in \mathcal{X}\right\}$. The conditionally $\epsilon$-typical set relative to $Q_{X Y}$ is denoted as $\mathcal{T}_{\epsilon}^{n}\left(Q_{X Y} \mid x^{n}\right):=\left\{y^{n} \in \mathcal{X}^{n}:\left(x^{n}, y^{n}\right) \in \mathcal{T}_{\epsilon}^{n}\left(Q_{X Y}\right)\right\}$. For brevity, we sometimes write $\mathcal{T}_{\epsilon}^{n}\left(Q_{X}\right)$ and $\mathcal{T}_{\epsilon}^{n}\left(Q_{X Y} \mid x^{n}\right)$ as $\mathcal{T}_{\epsilon}^{n}$ and $\mathcal{T}_{\epsilon}^{n}\left(x^{n}\right)$ respectively. Other notation generally follows the book by Csiszár and Körner [31].

The total variation distance between two probability mass functions $P$ and $Q$ with a common alphabet $\mathcal{X}$ is defined by

$$
|P-Q|:=\frac{1}{2} \sum_{x \in \mathcal{X}}|P(x)-Q(x)|
$$

By the definition of $\epsilon$-typical set, we have that for any $x^{n} \in$ $\mathcal{T}_{\epsilon}^{n}\left(Q_{X}\right),\left|T_{x^{n}}-Q_{X}\right| \leq \frac{\epsilon}{2}$.

Fix distributions $P_{X}, Q_{X} \in \mathcal{P}(\mathcal{X})$. Then the relative entropy and the Rényi divergence of order $1+s \in(0,1) \cup(1, \infty)$ are respectively defined as

$$
\begin{aligned}
D\left(P_{X} \| Q_{X}\right) & :=\sum_{x \in \mathcal{X}} P_{X}(x) \log \frac{P_{X}(x)}{Q_{X}(x)}, \quad \text { and } \\
D_{1+s}\left(P_{X} \| Q_{X}\right) & :=\frac{1}{s} \log \sum_{x \in \mathcal{X}} P_{X}(x)^{1+s} Q_{X}(x)^{-s}
\end{aligned}
$$

and the conditional versions are respectively defined as

$$
\begin{gathered}
D\left(P_{Y \mid X} \| Q_{Y \mid X} \mid P_{X}\right):=D\left(P_{X} P_{Y \mid X} \| P_{X} Q_{Y \mid X}\right) \\
D_{1+s}\left(P_{Y \mid X} \| Q_{Y \mid X} \mid P_{X}\right):=D_{1+s}\left(P_{X} P_{Y \mid X} \| P_{X} Q_{Y \mid X}\right)
\end{gathered}
$$

where throughout, $\log$ is to the natural base e and $s \geq-1$.The Rényi divergences of order 0,1 , and $\infty$ are respectively defined as

$$
\begin{aligned}
D_{0}\left(P_{X} \| Q_{X}\right) & :=\lim _{s \downarrow-1} D_{1+s}\left(P_{X} \| Q_{X}\right) \\
& =-\log \left\{Q_{X}\left(P_{X}>0\right)\right\} ; \\
D_{1}\left(P_{X} \| Q_{X}\right) & :=\lim _{s \rightarrow 0} D_{1+s}\left(P_{X} \| Q_{X}\right) \\
& =D\left(P_{X} \| Q_{X}\right) ; \\
D_{\infty}\left(P_{X} \| Q_{X}\right) & :=\lim _{s \rightarrow \infty} D_{1+s}\left(P_{X} \| Q_{X}\right) \\
& =\log _{x} \sup _{x} \frac{P_{X}(x)}{Q_{X}(x)} .
\end{aligned}
$$

Hence a special case of the Rényi divergence is the usual relative entropy.

Finally, we write $f(n) \dot{\leq} g(n)$ if $\limsup _{n \rightarrow \infty} \frac{1}{n} \log \frac{f(n)}{g(n)} \leq$ 0 . In addition, $f(n) \doteq g(n)$ means $f(n) \leq g(n)$ and $g(n) \dot{\leq}$ $f(n)$. We use $o(1), \delta_{n}, \delta_{n}^{\prime}, \delta_{n}^{\prime \prime}$ to denote generic sequences tending to zero as $n \rightarrow \infty$. For $a \in \mathbb{R},[a]^{+}:=\max \{a, 0\}$ denotes positive clipping.

\section{Problem Formulation}

We consider the channel resolvability problem illustrated in Fig. 1. Given a channel $P_{Y \mid X}$ and a target distribution $Q_{Y}$, we wish to minimize the alphabet size of a random variable $M_{n}$ that is uniformly distributed over ${ }^{1} \mathcal{M}_{n}:=\left\{1, \ldots, \mathrm{e}^{n R}\right\}$ ( $R$ is a positive number known as the rate), such that given common randomness $\mathcal{C}_{n}$, the output distribution

$$
P_{Y^{n} \mid \mathcal{C}_{n}}\left(y^{n} \mid c_{n}\right):=\frac{1}{\left|\mathcal{M}_{n}\right|} \sum_{m \in \mathcal{M}_{n}} \prod_{i=1}^{n} P_{Y \mid X}\left(y_{i} \mid f_{c_{n}, i}(m)\right)
$$

forms a good approximation to the product distribution $Q_{Y}^{n}$. Here $\mathcal{C}_{n}$ is a random variable independent of the random variable $M_{n}$. If we set $\mathcal{C}_{n}=\left\{X^{n}(m)\right\}_{m \in \mathcal{M}_{n}}$ with $X^{n}(m) \sim$ $P_{X^{n}}$ for all $m \in \mathcal{M}_{n}$, and set $f_{\mathcal{C}_{n}}(m)=X^{n}(m)$, then the random mapping is known as a conventional random code. If the input distribution is i.i.d., i.e., $P_{X^{n}}=P_{X}^{n}$, then it is known as an i.i.d. random code. In contrast to previous works on the channel resolvability problem [2], here we employ the Rényi divergence

$$
D_{1+s}\left(P_{Y^{n} \mathcal{C}_{n}} \| Q_{Y}^{n} P_{\mathcal{C}_{n}}\right)
$$

to measure the discrepancy between $P_{Y^{n}}$ and $Q_{Y}^{n}$.

Observe that

$$
\begin{aligned}
& \mathrm{e}^{s D_{1+s}\left(P_{Y^{n}} \mathcal{C}_{n} \| Q_{Y}^{n} P_{\mathcal{C}_{n}}\right)} \\
& =\mathbb{E}_{\mathcal{C}_{n}}\left[\sum_{y^{n}} \sum_{m} P(m) P\left(y^{n} \mid f_{\mathcal{C}_{n}}(m)\right)\right. \\
& \left.\quad \times\left(\frac{\sum_{m} P(m) P\left(y^{n} \mid f_{\mathcal{C}_{n}}(m)\right)}{Q\left(y^{n}\right)}\right)^{s}\right] .
\end{aligned}
$$

Hence to guarantee that $D_{1+s}\left(P_{Y^{n} \mathcal{C}_{n}} \| Q_{Y}^{n} P_{\mathcal{C}_{n}}\right)$ is finite for $s \geq 0$, we assume $P_{Y \mid X=x} \ll Q_{Y}$ for all $x \in \mathcal{X}$; otherwise, we can remove all the values $x$ such that $P_{Y \mid X=x} \nless Q_{Y}$ from $\mathcal{X}$. However, it is worth noting that we do not need to do so for $-1 \leq s<0$, since $D_{1+s}\left(P_{Y^{n} \mathcal{C}_{n}} \| Q_{Y}^{n} P_{\mathcal{C}_{n}}\right)$ is always

\footnotetext{
${ }^{1}$ For simplicity, we assume that $\mathrm{e}^{n R}$ and similar expressions (such as $\mathrm{e}^{R}$ ) are integers.
} 


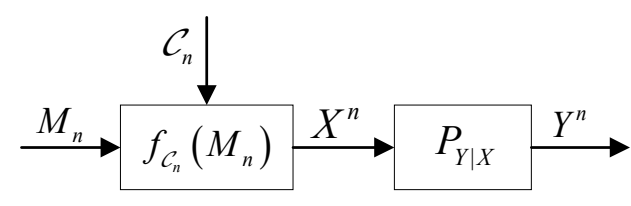

Fig. 1. Channel resolvability problem: $\mathcal{C}_{n}$ is independent of the message $M_{n} \in \mathcal{M}_{n}$, and $f_{\mathcal{C}_{n}}$ is a random function (induced by $\mathcal{C}_{n}$ ).

finite regardless of whether $P_{Y \mid X=x} \ll Q_{Y}$ for all $x \in \mathcal{X}$ or $P_{Y \mid X=x} \nless Q_{Y}$ for some $x \in \mathcal{X}$. Furthermore, for simplicity, for the case $s=-1$ we assume $P_{Y \mid X=x} \gg Q_{Y}$ for some $x \in \mathcal{X}{ }^{2}$ Hence $D_{0}\left(P_{Y^{n} \mathcal{C}_{n}} \| Q_{Y}^{n} P_{\mathcal{C}_{n}}\right)=0$ if the channel input is fixed to $x^{n}$.

Traditionally, the code $\mathcal{C}_{n}$ is deterministic and so the measure $D_{1+s}\left(P_{Y^{n}} \| Q_{Y}^{n}\right)$ is analyzed. However, in our setting, especially in Section II-D, we are interested in questions concerning the ensemble performance of random codes $\mathcal{C}_{n}$. Hence, we analyze the discrepancy measure $D_{1+s}\left(P_{Y^{n} \mathcal{C}_{n}} \| Q_{Y}^{n} P_{\mathcal{C}_{n}}\right)$, which represents the conditional Rényi divergence between the simulated and target distributions given the random code $\mathcal{C}_{n}$. Besides, we are also interested in another related discrepancy measure $^{3} \mathbb{E}_{\mathcal{C}_{n}}\left[D_{1+s}\left(P_{Y^{n} \mid \mathcal{C}_{n}} \| Q_{Y}^{n}\right)\right]$. Since for $s=0$,

$$
\begin{aligned}
\mathbb{E}_{\mathcal{C}_{n}}\left[D_{1+s}\left(P_{Y^{n} \mid \mathcal{C}_{n}} \| Q_{Y}^{n}\right)\right] & =D_{1+s}\left(P_{Y^{n}} \mathcal{C}_{n} \| Q_{Y}^{n} P_{\mathcal{C}_{n}}\right) \\
& =D\left(P_{Y^{n}} \mathcal{C}_{n} \| Q_{Y}^{n} P_{\mathcal{C}_{n}}\right),
\end{aligned}
$$

these measures are consistent with the one used in Parizi et al.'s paper [12] (which is $D\left(P_{Y^{n} \mathcal{C}_{n}} \| Q_{Y}^{n} P_{\mathcal{C}_{n}}\right)$ ). Furthermore, the three measures above satisfy the relationship

$$
\begin{aligned}
\inf _{f} D_{1+s}\left(P_{Y^{n}} \| Q_{Y}^{n}\right) & \leq \inf _{f_{\mathcal{C}_{n}}} \mathbb{E}_{\mathcal{C}_{n}}\left[D_{1+s}\left(P_{Y^{n}} \mid \mathcal{C}_{n} \| Q_{Y}^{n}\right)\right] \\
& \leq \inf _{f_{\mathcal{C}_{n}}} D_{1+s}\left(P_{Y^{n} \mathcal{C}_{n}} \| Q_{Y}^{n} P_{\mathcal{C}_{n}}\right) .
\end{aligned}
$$

In fact, for the achievability parts, we bound $\inf _{f_{\mathcal{C}_{n}}} D_{1+s}\left(P_{Y^{n} \mathcal{C}_{n}} \| Q_{Y}^{n} P_{\mathcal{C}_{n}}\right)$ from above and for the converse parts, we bound $\inf _{f} D_{1+s}\left(P_{Y^{n}} \| Q_{Y}^{n}\right)$ from below. This implies, by the chain of inequalities above, that our results in this paper hold for all these three measures.

\section{Main Results}

\section{A. One-Shot/Finite Blocklength Bounds}

We first consider the one-shot (i.e., blocklength $n$ equal to 1) or finite blocklength version of the problem. For this case, we provide several bounds in the following two lemmas, the proofs of which are given in Appendix B. These one-shot bounds will be used to derive asymptotic results in the next subsection. We believe that similar techniques used to obtain these bounds can be employed to derive second-order results, just as in [26], [27].

Lemma 1 (One-Shot Bounds for Direct Part). Consider a random mapping $f_{\mathcal{C}}: \mathcal{M}=\left\{1, \ldots, \mathrm{e}^{R}\right\} \rightarrow \mathcal{X}$. We set $\mathcal{C}=$ $\{X(m)\}_{m \in \mathcal{M}}$ with $X(m), m \in \mathcal{M}$ drawn independently for different $m$ 's and according to a same distribution $P_{X}$, and set

\footnotetext{
${ }^{2}$ Note that this condition is missing in the conference version [1].

${ }^{3}$ Here we would like to thank Prof. Masahito Hayashi for inspiring us to consider the measure $\mathbb{E}_{\mathcal{C}_{n}}\left[D_{1+s}\left(P_{Y^{n} \mid \mathcal{C}_{n}} \| Q_{Y}^{n}\right)\right]$.
}

$f_{\mathcal{C}}(m)=X(m)$. This forms a random code. For this random code, we have for $s \in[0,1]$ and any distribution $Q_{Y}$,

$$
\begin{aligned}
& \mathrm{e}^{s D_{1+s}\left(P_{Y \mathcal{C}} \| Q_{Y} P_{\mathcal{C}}\right)} \\
& \leq \mathrm{e}^{s D_{1+s}\left(P_{X Y} \| P_{X} Q_{Y}\right)-s R}+\mathrm{e}^{s D_{1+s}\left(P_{Y} \| Q_{Y}\right)} \\
& \leq 2 \mathrm{e}^{s \Gamma_{1+s}\left(P_{X}, P_{Y \mid X}, Q_{Y}, R\right)}
\end{aligned}
$$

where

$$
\begin{aligned}
& \Gamma_{1+s}\left(P_{X}, P_{Y \mid X}, Q_{Y}, R\right) \\
& :=\max \left\{D_{1+s}\left(P_{X Y} \| P_{X} Q_{Y}\right)-R, D_{1+s}\left(P_{Y} \| Q_{Y}\right)\right\} .
\end{aligned}
$$

In the other direction with $s \in[0,1)$, we have for any distribution $Q_{Y}$,

$$
\begin{aligned}
& \mathrm{e}^{-s D_{1-s}\left(P_{Y \mathcal{C}} \| Q_{Y} P_{\mathcal{C}}\right)} \\
& \geq 2^{-s}\left[\mathrm{e}^{s R} \sum_{x, y} P(x) P^{1-s}(y \mid x) Q^{s}(y) 1\left\{\frac{P(y \mid x)}{P(y)} \geq \mathrm{e}^{R}\right\}\right. \\
& \left.\quad+\sum_{x, y} P(x) P(y \mid x) P^{-s}(y) Q^{s}(y) 1\left\{\frac{P(y \mid x)}{P(y)}<\mathrm{e}^{R}\right\}\right] .
\end{aligned}
$$

Remark 1. A similar result to (19) was shown by Hayashi and Matsumoto [29, Thm. 14], but their result is a special case of ours with the setting $Q_{Y}=P_{Y}$.

Remark 2. Since in the proof we require Lemma 6 (see (93)) and the fact that $x \mapsto x^{s}$ is a concave function (see (99)), the proof does not apply to the case in which the Rényi divergence is of order $>2$.

Lemma 2 (One-Shot Bounds for Converse Part). For any deterministic mapping $f: \mathcal{M}=\left\{1, \ldots, \mathrm{e}^{R}\right\} \rightarrow \mathcal{X}$ and any $s \in[0, \infty]$, we have for any distribution $Q_{Y}$,

$$
\mathrm{e}^{s D_{1+s}\left(P_{Y} \| Q_{Y}\right)} \geq \mathrm{e}^{s \Gamma_{1+s}\left(P_{X}, P_{Y \mid X}, Q_{Y}, R\right)}
$$

where

$$
\begin{aligned}
& P(x):=\sum_{m} P(m) 1\{f(m)=x\} \\
& P(y):=\sum_{x} P(x) P(y \mid x)
\end{aligned}
$$

respectively denote the distributions of $X$ and $Y$ induced by the mapping $f$, and $\Gamma_{1+s}\left(P_{X}, P_{Y \mid X}, Q_{Y}, R\right)$ is given by (21). In the other direction with $s \in[0,1)$, we have for any distribution $Q_{Y}$,

$$
\begin{aligned}
& \mathrm{e}^{-s D_{1-s}\left(P_{Y} \| Q_{Y}\right)} \\
& \leq \mathrm{e}^{s R} \sum_{x, y} P(x) P^{1-s}(y \mid x) Q^{s}(y) 1\left\{\frac{P(y \mid x)}{P(y)} \geq \frac{\mathrm{e}^{R}}{2}\right\} \\
& \quad+\sum_{x, y} P(x) P(y \mid x) P^{-s}(y) Q^{s}(y) 1\left\{\frac{P(y \mid x)}{P(y)}<\frac{\mathrm{e}^{R}}{2}\right\}
\end{aligned}
$$

where $P_{X}$ and $P_{Y}$ are given in (24)-(25).

Remark 3. Note that the direct and converse parts for the $1+s$ case only differ by a factor of 2 . Similarly, the direct and converse parts for the $1-s$ case differ by a factor of $2^{-s}$ and $R$ is replaced by $R-\log 2$. 
Remark 4. For any random mapping $f_{\mathcal{C}}: \mathcal{M}=$ $\left\{1, \ldots, \mathrm{e}^{R}\right\} \rightarrow \mathcal{X}$, observe that for $s \in[0, \infty]$,

$$
\begin{aligned}
\mathrm{e}^{s D_{1+s}\left(P_{Y \mathcal{C}} \| Q_{Y} \times P_{\mathcal{C}}\right)} & =\mathbb{E}_{\mathcal{C}} \sum_{y} P^{1+s}(y) Q^{s}(y) \\
& \geq \min _{c} \sum_{y} P^{1+s}(y \mid c) Q^{s}(y) \\
& =\min _{c} \mathrm{e}^{s D_{1+s}\left(P_{Y \mid \mathcal{C}=c} \| Q_{Y}\right)},
\end{aligned}
$$

and for $s \in[0,1)$,

$$
\mathrm{e}^{-s D_{1-s}\left(P_{Y \mathcal{C}} \| Q_{Y} \times P_{\mathcal{C}}\right)} \leq \max _{c} \mathrm{e}^{-s D_{1-s}\left(P_{Y \mid \mathcal{C}=c} \| Q_{Y}\right)} .
$$

Therefore, the one-shot bounds in (23) and (26) still hold for any random codes.

Remark 5. By checking our proofs, it can be seen that Lemmas 1 and 2 hold not only for channels with finite (input and output) alphabets, but also for channels with countably infinite or continuous alphabets (e.g., Gaussian channels).

\section{B. Asymptotic Expressions}

We now consider the asymptotics of the Rényi divergence as the blocklength $n$ tends to infinity. The one-shot bounds can be used to prove the following theorem, in which the asymptotics of the Rényi divergences are characterized by multi-letter expressions. The proof of this theorem is provided in Appendix C.

Proposition 1 (Multi-letter Characterization). For any $s \in$ $[0,1]$, we have

$\frac{1}{n} \inf _{f_{\mathcal{C}_{n}}} D_{1+s}\left(P_{Y^{n} \mathcal{C}_{n}} \| Q_{Y}^{n} P_{\mathcal{C}_{n}}\right)=\Gamma_{1+s}^{(n)}\left(P_{Y \mid X}, Q_{Y}, R\right)+o(1)$

where

$$
\begin{aligned}
& \Gamma_{1+s}^{(n)}\left(P_{Y \mid X}, Q_{Y}, R\right):=\inf _{P_{X} n} \max \{ \\
& \left.\frac{1}{n} D_{1+s}\left(P_{X^{n} Y^{n}} \| P_{X^{n}} Q_{Y}^{n}\right)-R, \frac{1}{n} D_{1+s}\left(P_{Y^{n}} \| Q_{Y}^{n}\right)\right\} .
\end{aligned}
$$

Furthermore, for any $s \in(0,1)$, and any fixed positive integer $k$, we have

$$
\begin{aligned}
& \Gamma_{1-s}^{(n)}\left(P_{Y \mid X}, Q_{Y}, R\right)+o(1) \\
& \leq \frac{1}{n} \inf _{f_{\mathcal{C}_{n}}} D_{1-s}\left(P_{Y^{n} \mathcal{C}_{n}} \| Q_{Y}^{n} P_{\mathcal{C}_{n}}\right) \\
& \leq \Gamma_{1-s}^{(k)}\left(P_{Y \mid X}, Q_{Y}, R\right)+o(1),
\end{aligned}
$$

where in (34), o (1) is a term depending on both $n$ and $k$, and vanishing as $n \rightarrow \infty$ for any fixed $k$, and

$$
\begin{aligned}
& \Gamma_{1-s}^{(n)}\left(P_{Y \mid X}, Q_{Y}, R\right):=\inf _{P_{X^{n}}} \max _{t \in[0, s]}\left\{-\frac{t}{s} R\right. \\
& \left.-\frac{1}{n s} \log \sum_{x^{n}, y^{n}} P\left(x^{n}, y^{n}\right) P^{-t}\left(y^{n} \mid x^{n}\right) P^{t-s}\left(y^{n}\right) Q^{s}\left(y^{n}\right)\right\} .
\end{aligned}
$$

The infima in (31) and (34) are achieved by a sequence of random codes described in Lemma 1.
Remark 6. The converse part in (31) also holds for $s \in(1, \infty]$. That is, for any $s \in(1, \infty]$,

$\frac{1}{n} \inf _{f_{\mathcal{C}_{n}}} D_{1+s}\left(P_{Y^{n} \mathcal{C}_{n}} \| Q_{Y}^{n} P_{\mathcal{C}_{n}}\right) \geq \Gamma_{1+s}^{(n)}\left(P_{Y \mid X}, Q_{Y}, R\right)+o(1)$.

Remark 7. Note that in (33) and (34), the lower bound and the upper bounds differ only in the parameter of $\Gamma_{1-s}^{(\cdot)}$.

Remark 8. Proposition 1 holds even when the alphabets are not necessarily discrete.

Remark 9. From the definition of $\Gamma_{1+s}^{(n)}\left(P_{Y \mid X}, Q_{Y}, R\right)$, we have

$$
\begin{aligned}
& \Gamma_{1+s}^{(n)}\left(P_{Y \mid X}, Q_{Y}, R\right)=\inf _{P_{X^{n}}} \max _{t \in[0, s]}\left\{-\frac{t}{s} R\right. \\
& \left.+\frac{1}{n s} \log \sum_{x^{n}, y^{n}} P\left(x^{n}, y^{n}\right) P^{t}\left(y^{n} \mid x^{n}\right) P^{s-t}\left(y^{n}\right) Q^{-s}\left(y^{n}\right)\right\} .
\end{aligned}
$$

Therefore, the notations $\Gamma_{1+s}^{(n)}$ and $\Gamma_{1-s}^{(n)}$ are consistent in the sense that if we set $s$ to be $-s$ in $\Gamma_{1+s}^{(n)}$, we obtain $\Gamma_{1-s}^{(n)}$. That is to say, $\Gamma_{1+s}^{(n)}$ for $s \in[0, \infty]$ and $\Gamma_{1-s}^{(n)}$ for $s \in(0,1)$ can be unified as in (37) for $s \in(-1, \infty]$.

Next, the asymptotics of the Rényi divergence is characterized by single-letter expressions. We have an exact/tight result when the Rényi parameter $\in[1,2]$ and upper and lower bounds when the Rényi parameter $\in(0,1)$. This result is proved in Appendix D.

Theorem 1 (Asymptotics of Rényi Divergence). For any $s \in$ $[0,1]$, we have

$$
\begin{aligned}
\lim _{n \rightarrow \infty} \frac{1}{n} \inf _{f_{\mathcal{C}_{n}}} D_{1+s}\left(P_{Y^{n} \mathcal{C}_{n}} \| Q_{Y}^{n} P_{\mathcal{C}_{n}}\right) \\
=\min _{\widetilde{P}_{X}} \max \left\{\sum_{x} \widetilde{P}_{X}(x) D_{1+s}\left(P_{Y \mid X}(\cdot \mid x) \| Q_{Y}\right)-R,\right. \\
\max _{\left.\widetilde{P}_{Y \mid X} \eta_{1+s}\left(P_{Y \mid X}, Q_{Y}, \widetilde{P}_{X}, \widetilde{P}_{Y \mid X}\right)\right\}}
\end{aligned}
$$

where

$$
\begin{aligned}
& \eta_{1+s}\left(P_{Y \mid X}, Q_{Y}, \widetilde{P}_{X}, \widetilde{P}_{Y \mid X}\right) \\
& :=\left(-\frac{1}{s}-1\right) D\left(\widetilde{P}_{Y \mid X} \| P_{Y \mid X} \mid \widetilde{P}_{X}\right)+D\left(\widetilde{P}_{Y} \| Q_{Y}\right) .
\end{aligned}
$$

For any $s \in(0,1)$, we have

$$
\begin{aligned}
& \Gamma_{1-s}^{\mathrm{LB}}\left(P_{Y \mid X}, Q_{Y}, R\right) \\
& \leq \liminf _{n \rightarrow \infty} \frac{1}{n} \inf _{f_{\mathcal{C}_{n}}} D_{1-s}\left(P_{Y^{n} \mathcal{C}_{n}} \| Q_{Y}^{n} P_{\mathcal{C}_{n}}\right) \\
& \leq \limsup _{n \rightarrow \infty} \frac{1}{n} \inf _{f_{\mathcal{C}_{n}}} D_{1-s}\left(P_{Y^{n} \mathcal{C}_{n}} \| Q_{Y}^{n} P_{\mathcal{C}_{n}}\right) \\
& \leq \Gamma_{1-s}^{\mathrm{UB}}\left(P_{Y \mid X}, Q_{Y}, R\right),
\end{aligned}
$$


where

$$
\begin{aligned}
& \Gamma_{1-s}^{\mathrm{LB}}\left(P_{Y \mid X}, Q_{Y}, R\right) \\
& :=\min _{\widetilde{P}_{X}, \widetilde{P}_{Y \mid X}} \max \left\{\left(\frac{1}{s}-1\right) D\left(\widetilde{P}_{Y \mid X} \| P_{Y \mid X} \mid \widetilde{P}_{X}\right)\right. \\
& +D\left(\widetilde{P}_{Y \mid X} \| Q_{Y} \mid \widetilde{P}_{X}\right)-R, \\
& \left.\left(\frac{1}{s}-1\right) D\left(\widetilde{P}_{Y \mid X} \| P_{Y \mid X} \mid \widetilde{P}_{X}\right)+D\left(\widetilde{P}_{Y} \| Q_{Y}\right)\right\} \text {, } \\
& \Gamma_{1-s}^{\mathrm{UB}}\left(P_{Y \mid X}, Q_{Y}, R\right) \\
& :=\min _{\widetilde{P}_{X}, \widetilde{P}_{Y \mid X}} \max \left\{\left(\frac{1}{s}-1\right) D\left(\widetilde{P}_{Y \mid X} \| P_{Y \mid X} \mid \widetilde{P}_{X}\right)\right. \\
& +D\left(\widetilde{P}_{Y \mid X} \| Q_{Y} \mid \widetilde{P}_{X}\right)-R, \\
& \frac{1}{s} D\left(\widetilde{P}_{Y \mid X} \| P_{Y \mid X} \mid \widetilde{P}_{X}\right)+D\left(\widetilde{P}_{Y} \| Q_{Y}\right) \\
& \left.-\min _{\widehat{P}_{Y \mid X}: \widehat{P}_{Y \mid X} \circ \widetilde{P}_{X}=\widetilde{P}_{Y \mid X} \circ \widetilde{P}_{X}} D\left(\widehat{P}_{Y \mid X} \| P_{Y \mid X} \mid \widetilde{P}_{X}\right)\right\} \text {. }
\end{aligned}
$$

We also have

$$
\lim _{n \rightarrow \infty} \frac{1}{n} \inf _{f_{\mathcal{C}_{n}}} D_{0}\left(P_{Y^{n} \mathcal{C}_{n}} \| Q_{Y^{n}} P_{\mathcal{C}_{n}}\right)=0
$$

Furthermore, the infima in (38) and $\Gamma_{1-s}^{\mathrm{UB}}\left(P_{Y \mid X}, Q_{Y}, R\right)$ are achieved by a sequence of constant composition codes.

Remark 10. Similar to Remark 6, the converse part in (38) also holds for $s \in(1, \infty]$. That is, for any $s \in(1, \infty]$,

$$
\begin{aligned}
\liminf _{n \rightarrow \infty} \frac{1}{n} \inf _{f_{\mathcal{C}_{n}}} D_{1+s}\left(P_{Y^{n} \mathcal{C}_{n}} \| Q_{Y}^{n} P_{\mathcal{C}_{n}}\right) \\
\geq \min _{\widetilde{P}_{X}} \max \left\{\sum_{x} \widetilde{P}_{X}(x) D_{1+s}\left(P_{Y \mid X}(\cdot \mid x) \| Q_{Y}\right)-R,\right. \\
\left.\max _{\widetilde{P}_{Y \mid X}} \eta_{1+s}\left(P_{Y \mid X}, Q_{Y}, \widetilde{P}_{X}, \widetilde{P}_{Y \mid X}\right)\right\}
\end{aligned}
$$

Remark 11. The expression in (38) for $s \in[0,1]$ and $\Gamma_{1-s}^{\mathrm{LB}}\left(P_{Y \mid X}, Q_{Y}, R\right)$ or $\Gamma_{1-s}^{\mathrm{UB}}\left(P_{Y \mid X}, Q_{Y}, R\right)$ for $s \in(-1,0)$ may appear to be inconsistent; however, this is not true. It can be easily shown that

$$
\begin{aligned}
& \sum_{x} \widetilde{P}_{X}(x) D_{1+s}\left(P_{Y \mid X}(\cdot \mid x) \| Q_{Y}\right) \\
& =\max _{\widetilde{P}_{Y \mid X}}\left\{\left(-\frac{1}{s}-1\right) D\left(\widetilde{P}_{Y \mid X} \| P_{Y \mid X} \mid \widetilde{P}_{X}\right)\right. \\
& \left.\quad+D\left(\widetilde{P}_{Y \mid X} \| Q_{Y} \mid \widetilde{P}_{X}\right)\right\} .
\end{aligned}
$$

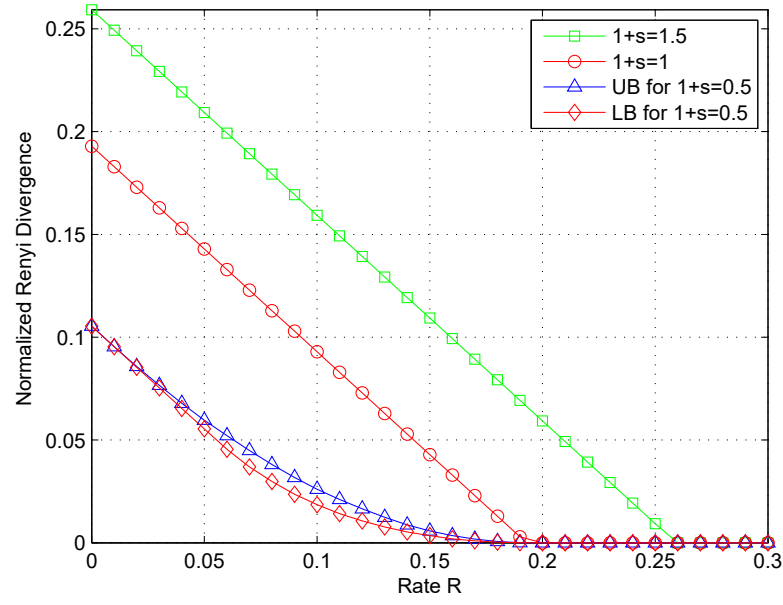

Fig. 2. Illustration of the Rényi divergence measure $\frac{1}{n} \inf _{f_{\mathcal{C}_{n}}} D_{1+s}\left(P_{Y^{n} \mathcal{C}_{n}} \| Q_{Y}^{n} P_{\mathcal{C}_{n}}\right)$ for $s \in[0,1]$ in (38) and the upper $\Gamma_{1-s}^{\mathrm{UB}}\left(P_{Y \mid X}, Q_{Y}, R\right)$ and lower bounds $\Gamma_{1-s}^{\mathrm{LB}}\left(P_{Y \mid X}, Q_{Y}, R\right)$ for $s \in(-1,0)$ in (43) and (44), for the BSC $Y=X \oplus V, V \sim \operatorname{Bern}(0.2)$ and the target distribution $Q_{Y}=\operatorname{Bern}(0.5)$.

Hence we can rewrite (38) as

$$
\begin{aligned}
& \lim _{n \rightarrow \infty} \frac{1}{n} \inf _{f_{\mathcal{C}_{n}}} D_{1+s}\left(P_{Y^{n} \mathcal{C}_{n}} \| Q_{Y^{n}} P_{\mathcal{C}_{n}}\right) \\
& =\min _{\widetilde{P}_{X}} \max _{\widetilde{P}_{Y \mid X}} \max \left\{\left(-\frac{1}{s}-1\right) D\left(\widetilde{P}_{Y \mid X} \| P_{Y \mid X} \mid \widetilde{P}_{X}\right)\right. \\
& \quad+D\left(\widetilde{P}_{Y \mid X} \| Q_{Y} \mid \widetilde{P}_{X}\right)-R, \\
& \left.\quad\left(-\frac{1}{s}-1\right) D\left(\widetilde{P}_{Y \mid X} \| P_{Y \mid X} \mid \widetilde{P}_{X}\right)+D\left(\widetilde{P}_{Y} \| Q_{Y}\right)\right\} .
\end{aligned}
$$

In other words, the expression in (38) for $s \in[0,1]$ is consistent with $\Gamma_{1-s}^{\mathrm{LB}}\left(P_{Y \mid X}, Q_{Y}, R\right)$ for $s \in(-1,0)$.

Note that $\Gamma_{1-s}^{\mathrm{UB}}\left(P_{Y \mid X}, Q_{Y}, R\right)$ and $\Gamma_{1-s}^{\mathrm{LB}}\left(P_{Y \mid X}, Q_{Y}, R\right)$ differ only in the second term in the maximization. Moreover, when $R$ is large enough, they are both equal to zero; see Theorem 2 in the next subsection.

We numerically calculate the asymptotics of the normalized Rényi divergence for binary symmetric channel (BSC) $Y=X \oplus V, V \sim \operatorname{Bern}(0.2)$ and $Q_{Y}=\operatorname{Bern}(0.5)$, and display the result in Fig. 2. From this figure, we observe that the normalized Rényi divergence decays as $R$ increases, and finally vanishes for large enough $R$. Moreover, the rate at which the normalized Rényi divergence transitions from a positive quantity to zero increases in $s$ for the Rényi parameter $1+s \in[1,2]$, and remains the same when $1+s \in(0,1]$. A rigorous statement of this point will be provided in the next subsection.

\section{Rényi Resolvability}

We now compute the Rényi resolvability, which is defined as the minimum rate $R$ of the input process $\left\{X^{n}(m)\right.$ : $\left.m \in \mathcal{M}_{n}\right\}$ to ensure that the unnormalized Rényi divergence 
$D_{1+s}\left(P_{Y^{n} \mathcal{C}_{n}} \| Q_{Y}^{n} P_{\mathcal{C}_{n}}\right)$ or the normalized Rényi divergence $\frac{1}{n} D_{1+s}\left(P_{Y^{n} \mathcal{C}_{n}} \| Q_{Y}^{n} P_{\mathcal{C}_{n}}\right)$ vanishes. We assume that

$$
\mathcal{P}\left(P_{Y \mid X}, Q_{Y}\right):=\left\{P_{X}: P_{Y \mid X} \circ P_{X}=Q_{Y}\right\} \neq \emptyset .
$$

Otherwise, there does not exist a code such that $\frac{1}{n} D_{1+s}\left(P_{Y^{n}} \mathcal{C}_{n} \| Q_{Y}^{n} P_{\mathcal{C}_{n}}\right) \quad$ vanishes. By Theorem 1 we easily obtain the following result. The proof is provided in Appendix E.

Theorem 2. (Rényi Resolvability) For $s \in[-1,1] \cup\{\infty\}$, we have $^{4}$

$$
\begin{aligned}
& \inf \left\{R: \inf _{\mathcal{C}_{n}} D_{1+s}\left(P_{Y^{n} \mathcal{C}_{n}} \| Q_{Y}^{n} P_{\mathcal{C}_{n}}\right) \rightarrow 0\right\} \\
& =\inf \left\{R: \frac{1}{n} \inf _{f_{\mathcal{C}_{n}}} D_{1+s}\left(P_{Y^{n} \mathcal{C}_{n}} \| Q_{Y}^{n} P_{\mathcal{C}_{n}}\right) \rightarrow 0\right\} \\
& =R_{1+s}\left(P_{Y \mid X}, Q_{Y}\right),
\end{aligned}
$$

where

$$
\begin{aligned}
& R_{1+s}\left(P_{Y \mid X}, Q_{Y}\right) \\
& := \begin{cases}\min _{P_{X} \in \mathcal{P}\left(P_{Y \mid X}, Q_{Y}\right)} \sum_{x} P_{X}(x) & s \in(0,1] \cup\{\infty\} \\
\quad \times D_{1+s}\left(P_{Y \mid X}(\cdot \mid x) \| Q_{Y}\right), & s \in(-1,0] \\
\min _{P_{X} \in \mathcal{P}\left(P_{Y \mid X}, Q_{Y}\right)} D\left(P_{Y \mid X} \| Q_{Y} \mid P_{X}\right), & s=-1 .\end{cases}
\end{aligned}
$$

Remark 12. Similar to Remarks 6 and 10, the converse part in (51) also holds for any $s \in(1, \infty)$. That is, for any $s \in(1, \infty)$,

$$
\begin{aligned}
& \inf \left\{R: \inf _{\mathcal{C}_{n}} D_{1+s}\left(P_{Y^{n} \mathcal{C}_{n}} \| Q_{Y}^{n} P_{\mathcal{C}_{n}}\right) \rightarrow 0\right\} \\
& \geq \inf \left\{R: \frac{1}{n} \inf _{f_{\mathcal{C}_{n}}} D_{1+s}\left(P_{Y^{n} \mathcal{C}_{n}} \| Q_{Y}^{n} P_{\mathcal{C}_{n}}\right) \rightarrow 0\right\} \\
& \geq \min _{P_{X} \in \mathcal{P}\left(P_{Y \mid X}, Q_{Y}\right)} \sum_{x} P_{X}(x) D_{1+s}\left(P_{Y \mid X}(\cdot \mid x) \| Q_{Y}\right) .
\end{aligned}
$$

Remark 13. The Rényi resolvabilities for the normalized or unnormalized Rényi divergence are the same.

Remark 14. Note that for the case $s \in(-1,0]$, $R_{1+s}\left(P_{Y \mid X}, Q_{Y}\right) \quad$ can be also expressed as $\min _{P_{X} \in \mathcal{P}\left(P_{Y \mid X}, Q_{Y}\right)} I(X ; Y)$ where $(X, Y) \sim P_{X} P_{Y \mid X}$, since $P_{X} \in \mathcal{P}\left(P_{Y \mid X}, Q_{Y}\right)$.

Remark 15. Since $\mathcal{P}\left(P_{Y \mid X}, Q_{Y}\right)$ is nonempty, $R_{1+s}\left(P_{Y \mid X}, Q_{Y}\right)$ is finite. Hence it can be shown $\lim _{s \downarrow 0} R_{1+s}\left(P_{Y \mid X}, Q_{Y}\right)=R_{1}\left(P_{Y \mid X}, Q_{Y}\right)$ (by using the continuity of Rényi divergence [35]). Hence $R_{1+s}\left(P_{Y \mid X}, Q_{Y}\right)$ is continuous in $s$ for $s \in(-1, \infty]$. See the bottom subfigure of Fig. 3 .

Remark 16. This result for the case $s=0$ and the normalized divergence (i.e., the normalized relative entropy) was first shown by Wyner [8] for stationary memoryless channels, and was extended to general channels by Han and Verdú

\footnotetext{
${ }^{4}$ We thank an anonymous reviewer for providing the achievability proof for the case $s=\infty$ in Appendix E-B2. Similar proof ideas can be found in [16], [32]-[34].
}
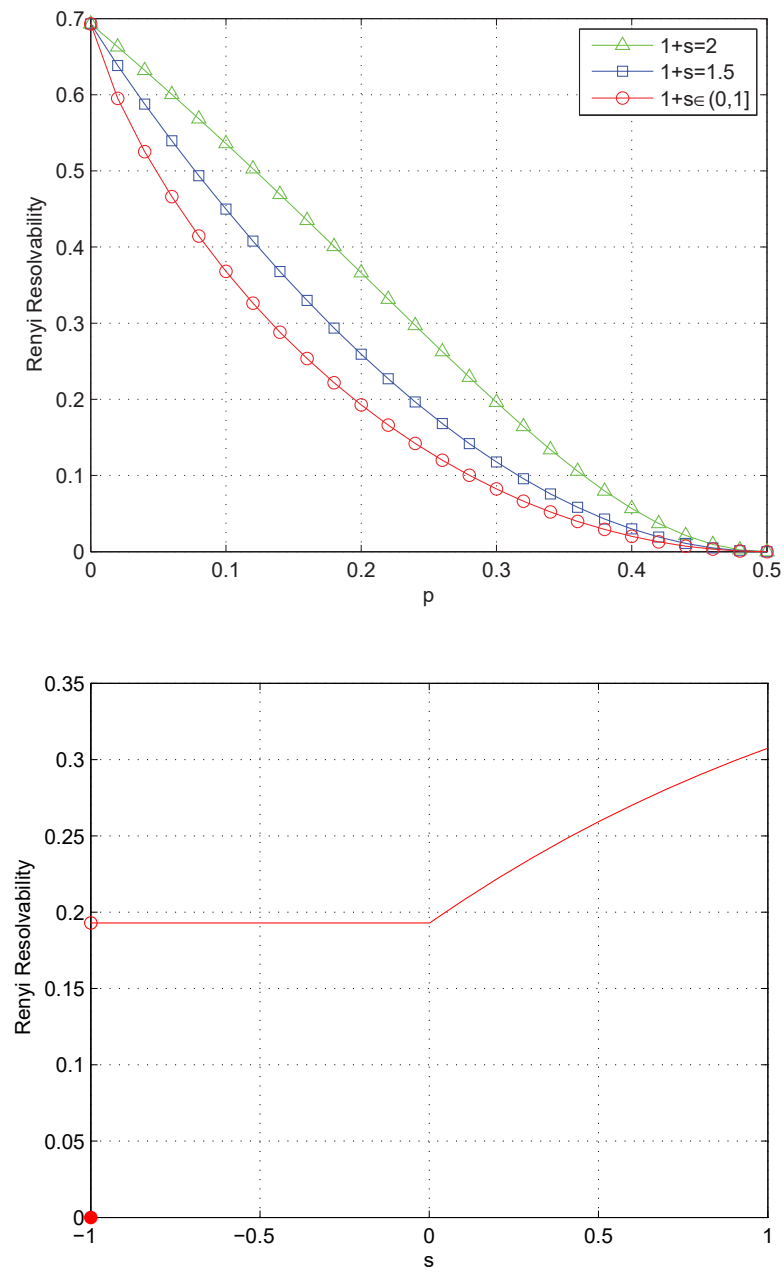

Fig. 3. Illustration of the Rényi resolvability in (51) for the BSC $Y=$ $X \oplus V, V \sim \operatorname{Bern}(p)$ and $Q_{Y}=\operatorname{Bern}(0.5)$. For the bottom subfigure, $p=0.2$.

[2]. Hence our result for the normalized divergence is an extension of theirs to the Rényi divergence $D_{1+s}$ of all orders $s \in[-1,1] \cup\{\infty\}$. For the normalized divergence, our results for $s \in(0,1] \cup\{\infty\}$ and converse parts for $s \in(-1,0)$ are new. The case $s=0$ and the unnormalized divergence (i.e., the unnormalized relative entropy case) has been shown in other works, such as those by Hayashi [3], [4], which also imply the achievability result part for $s \in(-1,0)$ (since the approximation measure $D_{1+s}$ for $s \in(-1,0)$ is weaker than $D_{1}$ ). By Pinsker's inequality for the Rényi divergence [35], the resolvability result under the TV distance measure [2] implies the converse for $s \in(-1,0]$. For the unnormalized divergence, our results for $s \in(0,1] \cup\{\infty\}$ are new. All the results above are summarized in Table I.

Remark 17. The first clause in (52) is the minimization of an expectation of Rényi divergences $\sum_{x} P_{X}(x) D_{1+s}\left(P_{Y \mid X}(\cdot \mid x) \| Q_{Y}\right)$ but it is not (and in general smaller than) the conventional conditional Rényi divergence $D_{1+s}\left(P_{X Y} \| P_{X} Q_{Y}\right)$ (see Verdú [36] or Fong and Tan [37]). An optimal i.i.d. code can achieve a rate 
TABLE I

SUMMARY OF RESULTS FOR NORMALIZED AND UNNORMALIZED RÉNYI RESOLVABILITY WITH DIFFERENT PARAMETERS.

\begin{tabular}{|l|c|c|}
\hline \multicolumn{2}{|c|}{ Cases } & Results \\
\hline \multirow{3}{*}{ Normalized Divergence } & $s=0$ & Wyner [8] and Han-Verdú [2] \\
\cline { 2 - 3 } & $s \in(-1,0)$ & Achievability: Wyner [8] and Han-Verdú [2]; Converse: Theorem 2 \\
\cline { 2 - 3 } & $s \in(0,1 \cup\{\infty\}$ & Theorem 2 \\
\hline \multirow{3}{*}{ Unnormalized Divergence } & $s=0$ & Combining Pinsker's inequality [3] [3] and Han-Verdú [2] \\
\cline { 2 - 3 } & $s \in(-1,0)$ & Theorem 2 \\
\cline { 2 - 3 } & $s \in(0,1] \cup\{\infty\}$ & Cond \\
\hline
\end{tabular}

equal to the minimization of conventional conditional Rényi divergence $D_{1+s}\left(P_{X Y} \| P_{X} Q_{Y}\right)$ [29, Thm. 14], while an optimal constant composition code for the normalized Rényi divergence or an optimal typical set code (a code with channel input distributed according to the target distribution $Q_{X}^{n}$ but truncated to an appropriate typical set) for both the unnormalized and normalized Rényi divergences can achieve a better (smaller) rate equal to the first clause in (52). This shows that the expectation of Rényi divergences also admits an operational interpretation as the minimum rate needed to drive the Rényi divergence to zero when its parameter is $\geq 1$. Besides, a similar definition for the conditional Rényi entropy can be found in [38], [39]. In addition, observe that any constant composition code can be approximated arbitrarily well by a typical set code by setting the typical set parameter $\epsilon$ arbitrarily close to 0 . But conversely, compared to typical set codes, constant composition codes are easier to analyze. This simplifies the proofs of our results significantly (e.g., that of Theorem 1). Furthermore, for constant composition codes, the codewords are each independently drawn from the uniform distribution on a type class. It is worth noting that in Han and Verdú s paper [2, Example 1], the extremal input process that results in the worst (largest) resolvability is also the uniform distribution on a type class.

The result in Theorem 2 for the BSC $Y=X \oplus V, V \sim$ $\operatorname{Bern}(p)$ and $Q_{Y}=\operatorname{Bern}(0.5)$ is illustrated in Fig. 3. For this case,

$$
\begin{aligned}
& R_{1+s}\left(P_{Y \mid X}, Q_{Y}\right) \\
& = \begin{cases}\log (2 \max \{p, \bar{p}\}) & s=\infty \\
\frac{1}{s} \log \left(p^{1+s} 2^{s}+\bar{p}^{1+s} 2^{s}\right) & s \in(0,1] \\
1-H_{2}(p) & s=(-1,0] \\
0 & s=-1\end{cases}
\end{aligned}
$$

\section{Exponential Behavior}

We now consider the exponent of $D_{1+s}\left(P_{Y^{n}} \mathcal{C}_{n} \| Q_{Y}^{n} P_{\mathcal{C}_{n}}\right)$ when the codebook is generated in an i.i.d. fashion. In this case, we can characterize the optimal exponent for this ensemble exactly. The proof of the following theorem is provided in Appendix F.

Theorem 3 (Exponential Behavior of i.i.d. Random Codes). Let $\mathcal{C}_{n}=\left\{X^{n}(m)\right\}_{m \in \mathcal{M}_{n}}$ with $X^{n}(m) \sim P_{X}^{n}, m \in \mathcal{M}_{n}$, and set $f_{\mathcal{C}_{n}}(m)=X^{n}(m)$, where $P_{X} \in \mathcal{P}\left(P_{Y \mid X}, Q_{Y}\right)$. For this i.i.d. code, if the rate $R$ satisfies for $s \in(0,1]$,

$$
R>D_{1+s}\left(P_{X Y} \| P_{X} \times Q_{Y}\right)
$$

and for $s \in(-1,0]$,

$$
R>D\left(P_{X Y} \| P_{X} \times Q_{Y}\right)=I(X ; Y),
$$

then we have

$\lim _{n \rightarrow \infty}-\frac{1}{n} \log D_{1+s}\left(P_{Y^{n} \mathcal{C}_{n}} \| Q_{Y}^{n} P_{\mathcal{C}_{n}}\right)=\mathrm{E}_{\mathrm{iid}}\left(P_{X}, P_{Y \mid X}, Q_{Y}\right)$,

where

$$
\begin{aligned}
& \mathrm{E}_{\mathrm{iid}}\left(P_{X}, P_{Y \mid X}, Q_{Y}\right) \\
& :=\left\{\begin{array}{ll}
\max _{t \in[s, 1]} t\left(R-D_{1+t}\left(P_{X Y} \| P_{X} \times Q_{Y}\right)\right) & s \in(0,1] \\
\max _{t \in[0,1]} t\left(R-D_{1+t}\left(P_{X Y} \| P_{X} \times Q_{Y}\right)\right) & s \in(-1,0]
\end{array} .\right.
\end{aligned}
$$

Remark 18. By checking the proof, we can obtain that for any $s \in(1, \infty]$, if $R>D_{1+s}\left(P_{X Y} \| P_{X} \times Q_{Y}\right)$, then the i.i.d. code above satisfies

$$
\begin{aligned}
& \liminf _{n \rightarrow \infty}-\frac{1}{n} \log D_{1+s}\left(P_{Y^{n} \mathcal{C}_{n}} \| Q_{Y}^{n} P_{\mathcal{C}_{n}}\right) \\
& \leq \sup _{t \geq 1} t\left(R-D_{1+t}\left(P_{X Y} \| P_{X} \times Q_{Y}\right)\right) .
\end{aligned}
$$

Remark 19. Similar to Remark 5, by checking our proofs, it can be seen that the achievability part in this theorem

$$
\lim _{n \rightarrow \infty}-\frac{1}{n} \log D_{1+s}\left(P_{Y^{n} \mathcal{C}_{n}} \| Q_{Y}^{n} P_{\mathcal{C}_{n}}\right) \geq \mathrm{E}_{\text {iid }}\left(P_{X}, P_{Y \mid X}, Q_{Y}\right),
$$

holds not only for channels with finite (input and output) alphabets, but also for channels with countably infinite or continuous alphabets (e.g., Gaussian channels). Hence Theorem 3 gives an exponential achievability result for channel resolvability problems with countable or continuous alphabets.

Remark 20. Observe that the exponent of i.i.d. random codes cannot be negative (see Lemma 1) and the exponent is nondecreasing in $R$. Hence for the i.i.d. code above with any rate $R>0$, we have

$$
\begin{aligned}
& \lim _{n \rightarrow \infty}-\frac{1}{n} \log D_{1+s}\left(P_{Y^{n}} \mathcal{C}_{n} \| Q_{Y}^{n} P_{\mathcal{C}_{n}}\right) \\
& =\widetilde{\mathrm{E}}_{\text {iid }}\left(P_{X}, P_{Y \mid X}, Q_{Y}\right),
\end{aligned}
$$

where

$$
\begin{aligned}
& \widetilde{\mathrm{E}}_{\mathrm{iid}}\left(P_{X}, P_{Y \mid X}, Q_{Y}\right) \\
& :=\left\{\begin{array}{ll}
{\left[\max _{t \in[s, 1]} t\left(R-D_{1+t}\left(P_{X Y} \| P_{X} \times Q_{Y}\right)\right)\right]^{+}} & s \in(0,1] \\
\max _{t \in[0,1]} t\left(R-D_{1+t}\left(P_{X Y} \| P_{X} \times Q_{Y}\right)\right) & s \in(-1,0]
\end{array} .\right.
\end{aligned}
$$



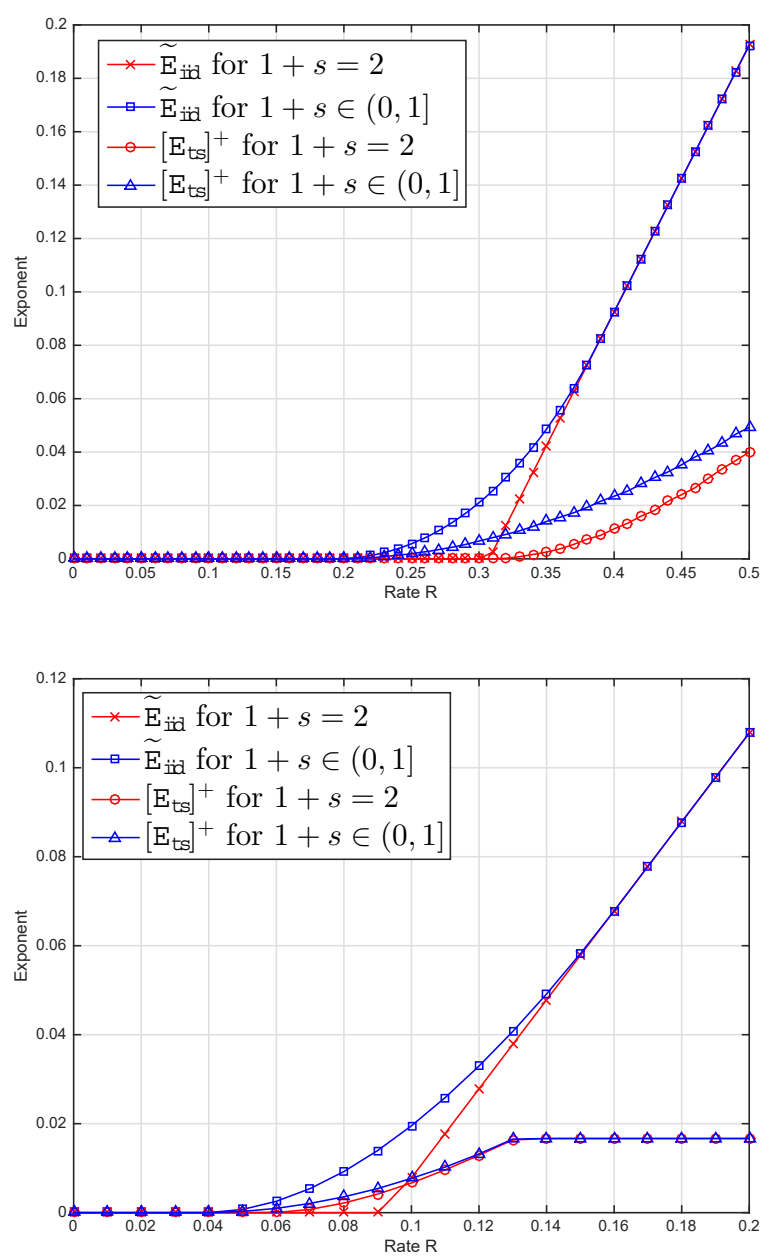

Fig. 4. Illustration of the exponent $\widetilde{\mathrm{E}}_{\mathrm{iid}}\left(P_{X}, P_{Y \mid X}, Q_{Y}\right)$ in (63) for the ensemble of i.i.d. random codes and the lower bound $\left[\mathrm{E}_{\mathrm{ts}}\left(P_{X}, P_{Y \mid X}, Q_{Y}\right)\right]^{+}$ in (66) on the exponent for the ensemble of typical set codes for Rényi parameter $1+s \in(0,2]$ for the BSC $Y=X \oplus V, V \sim \operatorname{Bern}(0.2)$. For the top subfigure, $Q_{Y}=\operatorname{Bern}(0.5)$, and for the bottom subfigure, $Q_{Y}=\operatorname{Bern}(0.23)$.

The result for $s=0$ (relative entropy) can be found in Parizi, Telatar and Merhav's paper [12]. The results for the other cases $s \in[-1,1] \backslash\{0\}$ are new. The result of Theorem 3 for a BSC is illustrated in Fig. 4.

Furthermore, for general codes, we show that the Rényi divergence decays at least exponentially fast, as long as the code rate is larger than the Rényi resolvability given in the previous subsection. The proof is provided in Appendix G.

Theorem 4 (General Lower Bound on the Rényi Divergence Exponent). Let $s \in[-1,1]$. If

$$
R>R_{1+s}\left(P_{Y \mid X}, Q_{Y}\right),
$$

then we have

$$
\begin{gathered}
\liminf _{n \rightarrow \infty}-\frac{1}{n} \log \inf _{f_{\mathcal{C}_{n}}}\left(D_{1+s}\left(P_{Y^{n} \mathcal{C}_{n}} \| Q_{Y}^{n} P_{\mathcal{C}_{n}}\right)\right) \\
\geq \max _{P_{X} \in \mathcal{P}\left(P_{Y \mid X}, Q_{Y}\right)} \max \left\{\widetilde{\mathrm{E}}_{\text {iid }}\left(P_{X}, P_{Y \mid X}, Q_{Y}\right),\right. \\
\left.\mathrm{E}_{\mathrm{ts}}\left(P_{X}, P_{Y \mid X}, Q_{Y}\right)\right\},
\end{gathered}
$$

where $\widetilde{\mathrm{E}}_{\mathrm{iid}}\left(P_{X}, P_{Y \mid X}, Q_{Y}\right)$ is defined in (63), and ${ }^{5}$

$$
\begin{aligned}
& \mathrm{E}_{\mathrm{ts}}\left(P_{X}, P_{Y \mid X}, Q_{Y}\right) \\
& := \begin{cases}\sup _{\epsilon \in(0,1]} \min \left\{\frac{\epsilon^{2} P_{\min }}{3}, \theta\left(s, \epsilon, P_{X}\right)\right\} & s \in(0,1] \\
\sup _{\epsilon \in(0,1]} \min \left\{\frac{\epsilon^{2} P_{\min }}{3}, \theta\left(0, \epsilon, P_{X}\right)\right\} & s \in(-1,0]\end{cases}
\end{aligned}
$$

with $P_{\min }:=\min _{x} P_{X}(x)$, and

$$
\begin{aligned}
& \theta\left(s, \epsilon, P_{X}\right) \\
& :=\sup _{t \in[s, 1]} t\left(R-(1+\epsilon) \sum_{x} P_{X}(x) D_{1+t}\left(P_{Y \mid X}(\cdot \mid x) \| Q_{Y}\right)\right) .
\end{aligned}
$$

Remark 21. From Theorem 4 and Remark 20, it can be easily observed that for $s \in(0,1]$, if $R_{1+s}\left(P_{Y \mid X}, Q_{Y}\right)<R<$ $\min _{P_{X} \in \mathcal{P}\left(P_{Y \mid X}, Q_{Y}\right)} D_{1+s}\left(P_{X Y} \| P_{X} \times Q_{Y}\right)$, then

$$
\begin{aligned}
& \max _{P_{X} \in \mathcal{P}\left(P_{Y \mid X}, Q_{Y}\right)} \mathrm{E}_{\mathrm{ts}}\left(P_{X}, P_{Y \mid X}, Q_{Y}\right) \\
& >\max _{P_{X} \in \mathcal{P}\left(P_{Y \mid X}, Q_{Y}\right)} \widetilde{\mathrm{E}}_{\mathrm{iid}}\left(P_{X}, P_{Y \mid X}, Q_{Y}\right) \\
& =0 .
\end{aligned}
$$

For this case, the optimal Rényi divergence of i.i.d. codes increases almost linearly as $n \rightarrow \infty$. This can be observed from the one-shot bounds (Lemmas 1 and 2). Hence i.i.d. codes are, in general, not optimal in achieving the best exponent for $s \in(0,1]$. This point is unsurprising given Remark 17, since as stated in Remark 17, optimal i.i.d. codes are not optimal in achieving even the Rényi resolvability. Hence, optimal i.i.d. codes are certainly not optimal in achieving the Rényi divergence exponent. This point can be seen from Fig. 4.

Remark 22. The optimal exponent of constant composition codes [31] has been studied by Parizi, Telatar, and Merhav [12], but different from our case, they consider the relative entropy between the channel output and the corresponding expected version (over the codebook) as the channel resolvability. Note that according to [12, Equation (13)], even in the $s=0$ case, this quantity is weaker than the Rényi divergence considered by us. They obtained ensemble tight results for constant composition and i.i.d. codes (and applied these results to the wiretap channel) but we are only able to do the same for the simpler i.i.d. codes.

\section{Application to the Wiretap Channel}

We apply the preceding results to the wiretap channel [40], [30]. In [13], Hou and Kramer proposed a new security measure, termed effective secrecy, for wiretap channels by exploiting the unnormalized KL divergence to quantify not only (the wiretapper's) confusion but also stealth. In this section, we generalize Hou and Kramer's result to a generalized divergence measure - the Rényi divergence. We provide a

\footnotetext{
${ }^{5}$ Here the subscript of $E_{t s}$ refers to typical set. The achievability scheme for this exponent is one with channel input following a truncated version of the target distribution $Q_{Y}^{n}$ to some typical set; hence we term this typical set code.
} 
complete characterization of the secrecy capacity region under this new and generalized leakage measure.

Consider a discrete memoryless wiretap channel $P_{Y Z \mid X}$, and two messages $\left(M_{0}, M_{1}\right)$ that are uniformly distributed over $\mathcal{M}_{0}:=\left\{1, \ldots, \mathrm{e}^{n R_{0}}\right\}$ and $\mathcal{M}_{1}:=\left\{1, \ldots, \mathrm{e}^{n R_{1}}\right\}$ respectively. A sender wants to transmit the pair $\left(M_{0}, M_{1}\right)$ to a legitimate user, and, at the same time, ensure that $M_{1}$ as almost independent from the wiretapper's observation $Z^{n}$.

Definition 1. An $\left(n, R_{0}, R_{1}\right)$ secrecy code is defined by two stochastic mappings $P_{X^{n} \mid M_{0} M_{1}}: \mathcal{M}_{0} \times \mathcal{M}_{1} \rightarrow \mathcal{X}^{n}$ and $P_{\widehat{M}_{0} \widehat{M}_{1} \mid Y^{n}}: \mathcal{Y}^{n} \rightarrow \mathcal{M}_{0} \times \mathcal{M}_{1}$

Given a target distribution $Q_{Z}$, we wish to maximize the alphabet size (or rate) of $M_{1}$ such that the distribution $P_{M_{1} Z^{n}}$ induced by the code is approximately equal to the target distribution $P_{M_{1}} Q_{Z}^{n}$ and $M_{1}$ can be decoded correctly asymptotically.

Definition 2. The tuple $\left(R_{0}, R_{1}\right)$ is $\left(Q_{Z}, 1+s\right)$-achievable if there exists a sequence of $\left(n, R_{0}, R_{1}\right)$ secrecy codes with induced distribution $P$ such that

1) Error constraint:

$$
\lim _{n \rightarrow \infty} \mathbb{P}\left(\left(M_{0}, M_{1}\right) \neq\left(\widehat{M}_{0}, \widehat{M}_{1}\right)\right)=0
$$

2) Secrecy constraint (generalized effective secrecy):

$$
\lim _{n \rightarrow \infty} D_{1+s}\left(P_{M_{1} Z^{n}} \| P_{M_{1}} Q_{Z}^{n}\right)=0 \text {. }
$$

It is worth noting that (71) is a generalized version of the notion of effective secrecy considered in [13]. Here we assume $Q_{Z}$ satisfies $\mathcal{P}\left(P_{Z \mid X}, Q_{Z}\right) \neq \emptyset\left(\mathcal{P}\left(P_{Z \mid X}, Q_{Z}\right)\right.$ is defined in (49)); otherwise, (71) cannot be satisfied by any secrecy code.

Definition 3. The $\left(Q_{Z}, 1+s\right)$-admissible region is defined as

$$
\mathcal{R}_{1+s}\left(Q_{Z}\right):=\text { Closure }\left\{Q_{Z} \text {-achievable }\left(R_{0}, R_{1}\right)\right\} \text {. }
$$

It is worth noting that our secrecy metric (even when $s=0)$ is stronger than the unnormalized relative entropy $D\left(P_{M_{1} Z^{n}} \| P_{M_{1}} P_{Z^{n}}\right)$ (or $\left.I\left(M_{1} ; Z^{n}\right)\right)$ considered in Bloch and Laneman [10], since

$$
\begin{aligned}
D\left(P_{M_{1} Z^{n}} \| P_{M_{1}} Q_{Z}^{n}\right) & =I\left(M_{1} ; Z^{n}\right)+D\left(P_{Z^{n}} \| Q_{Z}^{n}\right) \\
& \geq I\left(M_{1} ; Z^{n}\right) .
\end{aligned}
$$

For our secrecy metric, in addition to requiring that $M_{1}$ and $Z^{n}$ are approximately independent, we also require that the wiretapper's observation $Z^{n}$ is close to the product distribution $Q_{Z}^{n}$. This is similar to Hou and Kramer's work [13] and Csiszár and Narayan's work [41, Eqn. (6)], but we consider a continuum of secrecy measures indexed by $s \in[-1,1]$.

The interpretation of our secrecy measure with $s=0$ can be found in [13], where the authors interpreted $I\left(M_{1} ; Z^{n}\right)$ in (74) as a measure of "non-confusion" and $D\left(P_{Z^{n}} \| Q_{Z}^{n}\right)$ in (73) as a measure of "non-stealth". Under this interpretation, we set $Q_{Z}^{n}$ to be the distribution of the signal that the wiretapper observes if the sender is not sending useful information. Hence if the secrecy constraint (71) is satisfied then we can say that useful information is being transmitted stealthily.

\section{A. Main Result for Deterministic Encoders}

Before solving the problem, in this subsection we consider a simpler version of the problem-namely, a system with a deterministic encoder. That is, the encoder is restricted to a deterministic (non-stochastic) function $f: \mathcal{M}_{0} \times \mathcal{M}_{1} \rightarrow \mathcal{X}^{n}$ (denote the $\left(Q_{Z}, 1+s\right)$-admissible region for this case as $\mathcal{R}_{1+s}^{\text {det }}\left(Q_{Z}\right)$ ). Using Theorem 2 , we obtain the following theorem. The detailed proof is provided in Appendix $\mathrm{H}$.

Theorem 5. For $s \in[-1,1] \cup\{\infty\}$, we have

$$
\begin{aligned}
& \mathcal{R}_{1+s}^{\operatorname{det}}\left(Q_{Z}\right) \\
& =\bigcup_{P_{X} \in \mathcal{P}\left(P_{Z \mid X}, Q_{Z}\right)}\left\{\begin{array}{c}
\left(R_{0}, R_{1}\right): R_{0}+R_{1} \leq I(X ; Y) \\
R_{0} \geq \widetilde{R}_{1+s}\left(P_{X}, P_{Z \mid X}, Q_{Z}\right)
\end{array}\right\},
\end{aligned}
$$

where $\widetilde{R}_{1+s}\left(P_{X}, P_{Z \mid X}, Q_{Z}\right)$ is defined as

$$
\begin{aligned}
& \widetilde{R}_{1+s}\left(P_{X}, P_{Z \mid X}, Q_{Z}\right) \\
& :=\left\{\begin{array}{ll}
\sum_{x} P_{X}(x) D_{1+s}\left(P_{Z \mid X}(\cdot \mid x) \| Q_{Z}\right) & s \in(0,1] \cup\{\infty\} \\
D\left(P_{Z \mid X} \| Q_{Z} \mid P_{X}\right) & s \in(-1,0] \\
0 & s=-1
\end{array} .\right.
\end{aligned}
$$

Remark 23. This theorem provides an expression for the admissible rate region for the case with no extra randomness (i.e., the case with deterministic encoders). Related works on determining the amount of randomness needed to realize stochastic encoding include Watanabe and Oohama's work [42] and Chou, Vellambi, Bloch, and Kliewer's work [28]. Hence studying the deterministic encoder case is of independent interest.

Remark 24. Similar to the exponential behavior for the Rényi resolvability problem, it is easy to see that if $\left(R_{0}, R_{1}\right)$ is an interior point of $\mathcal{R}_{1+s}^{\mathrm{det}}\left(Q_{Z}\right)$, then the Rényi divergence for the wiretap channel with deterministic encoder also decays at least exponetially fast.

The result of Theorem 5 for the binary wiretap channel is illustrated in Fig. 5. From the figure (or the theorem), we observe that for the problem with deterministic encoder, the achievability of a rate pair $\left(R_{0}, R_{1}\right)$ does not necessarily imply the achievability of a rate pair $\left(R_{0}^{\prime}, R_{1}^{\prime}\right)$ such that $R_{0}^{\prime} \leq R_{0}, R_{1}^{\prime} \leq R_{1}$. This is because to meet the resolvability constraint, a certain amount of local randomness (besides the secret message $M_{1}$ ) at the sender is needed; this local randomness only comes from the non-secret message $M_{0}$ (since the encoder is a deterministic function of $M_{0}, M_{1}$ ). Therefore, a rate less than $R_{0}$ may not satisfy the resolvability constraint.

\section{B. Main Result for Stochastic Encoders}

If a stochastic encoder is allowed, we can add a virtual memoryless channel $P_{X \mid W}^{n}$ between the deterministic encoder and the channel. Then we have the following achievability result. 


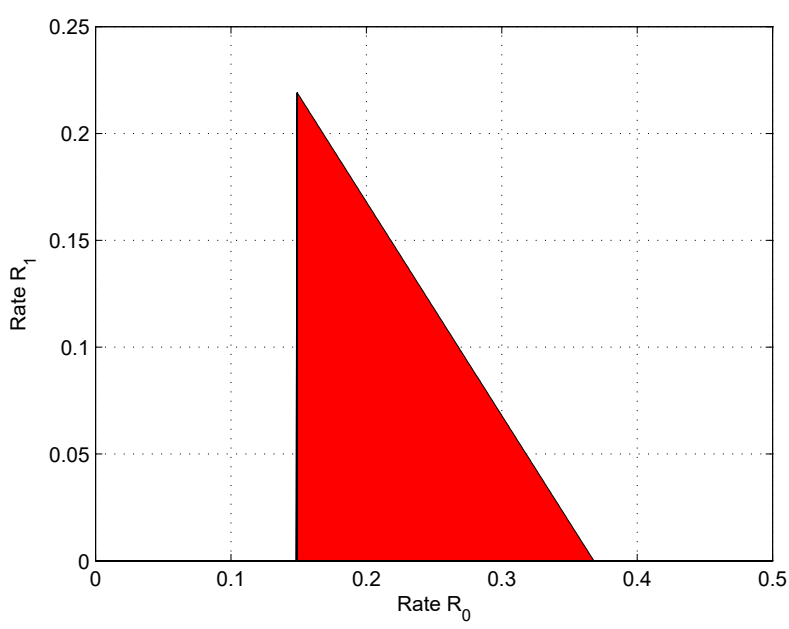

Fig. 5. Illustration of the admissible region for case of using a deterministic encoder and with Rényi parameter $1+s=2$ in (75) for the binary wiretap channel.

Proposition 2. For $s \in[-1,1] \cup\{\infty\}$, we have

$$
\begin{aligned}
& \mathcal{R}_{1+s}\left(Q_{Z}\right) \\
& \underset{\substack{P_{X W}: \\
P_{W} \in \mathcal{P}\left(P_{Z \mid W}, Q_{Z}\right)}}{\bigcup}\left\{\begin{array}{c}
\left(R_{0}, R_{1}\right): R_{0}+R_{1} \leq I(W ; Y), \\
R_{0} \geq \widetilde{R}_{1+s}\left(P_{W}, P_{Z \mid W}, Q_{Z}\right)
\end{array}\right\},
\end{aligned}
$$

where $\widetilde{R}_{1+s}\left(P_{W}, P_{Z \mid W}, Q_{Z}\right)$ is given by (76).

However, adding a memoryless channel is not optimal in general. In the following theorem, we completely characterize the admissible region, and show that adding a channel with memory between the encoder and channel is optimal. The proof of this theorem is given in Appendix I.

Theorem 6. For $s \in[-1,1] \cup\{\infty\}$, we have

$$
\begin{aligned}
& \mathcal{R}_{1+s}\left(Q_{Z}\right) \\
& =\underset{\substack{\widetilde{P}_{X} \in \mathcal{P}\left(P_{Z \mid X}, Q_{Z}\right) \\
\widetilde{P}_{X W}:}}{ }\left\{\begin{array}{c}
\left(R_{0}, R_{1}\right): R_{0}+R_{1} \leq I_{\widetilde{P}}(W ; Y) \\
R_{0} \geq \widetilde{R}_{1+s}^{\prime}\left(\widetilde{P}_{W \mid X} \widetilde{P}_{X}, P_{Z \mid X}, Q_{Z}\right)
\end{array}\right\} \\
& =\underset{\substack{\widetilde{P}_{X W}: \\
\widetilde{P}_{X} \in \mathcal{P}\left(P_{Z \mid X}, Q_{Z}\right)}}{\bigcup}\left\{\begin{array}{l}
\left(R_{0}, R_{1}\right): R_{0}+R_{1} \leq I_{\widetilde{P}}(W ; Y) \\
R_{1} \leq I_{\widetilde{P}}(W ; Y) \\
-\widetilde{R}_{1+s}^{\prime}\left(\widetilde{P}_{W \mid X} \widetilde{P}_{X}, P_{Z \mid X}, Q_{Z}\right)
\end{array}\right\},
\end{aligned}
$$

where $\widetilde{R}_{1+s}^{\prime}\left(\widetilde{P}_{W \mid X} \widetilde{P}_{X}, P_{Z \mid X}, Q_{Z}\right)$ is given by

$$
\widetilde{R}_{1+s}^{\prime}\left(\widetilde{P}_{W \mid X} \widetilde{P}_{X}, P_{Z \mid X}, Q_{Z}\right)
$$

$$
:=\left\{\begin{array}{cc}
\max _{\widetilde{P}_{Z \mid W X}\left\{-\frac{1+s}{s} D\left(\widetilde{P}_{Z \mid W X} \| P_{Z \mid X} \mid \widetilde{P}_{X W}\right)\right.} & \\
\left.+D\left(\widetilde{P}_{Z \mid W} \| Q_{Z} \mid \widetilde{P}_{W}\right)\right\}, & s \in(0,1] \\
I_{\widetilde{P}}(W ; Z), & \cup\{\infty\} \\
0, & s \in(-1,0] \\
& s=-1
\end{array}\right.
$$

Here $I_{\widetilde{P}}(W ; Y)$ in (78) and (79) and $I_{\widetilde{P}}(W ; Z)$ in (80) are the mutual informations evaluated under the distribution $\widetilde{P}_{W X} P_{Y Z \mid X}$. Furthermore, the ranges of $W$ in (78) and (79) may be assumed to satisfy $|\mathcal{W}| \leq|\mathcal{X}|+1$.

Remark 25. It is easy to show that if $R$ is an interior point of $\mathcal{R}_{1+s}\left(Q_{Z}\right)$, then the Rényi divergence for the wiretap channel problem with stochastic encoder also decays at least exponentially fast.

Remark 26 . We can define the effective secrecy capacity with the leakage measured by the Rényi divergence with parameter $1+s$ and with target output distribution $Q_{Z}$ as $C_{1+s}\left(Q_{Z}\right):=$ $\max _{\left(R_{0}, R_{1}\right) \in \mathcal{R}_{1+s}\left(Q_{Z}\right)} R_{1}$. The special case with $s=0$ was defined by Hou and Kramer [13], and they showed

$$
C_{1}\left(Q_{Z}\right)=\max _{\substack{\widetilde{P}_{X W}: \\ \widetilde{P}_{X} \in \mathcal{P}\left(P_{Z \mid X}, Q_{Z}\right)}}\left\{I_{\widetilde{P}}(W ; Y)-I_{\widetilde{P}}(W ; Z)\right\} .
$$

For the general case $s \in[-1,1] \cup\{\infty\}$, by Theorem 6 , we have

$$
\begin{aligned}
& C_{1+s}\left(Q_{Z}\right)= \\
& \max _{\substack{\widetilde{P}_{X W}: \\
\widetilde{P}_{X} \in \mathcal{P}\left(P_{Z \mid X}, Q_{Z}\right)}}\left\{I_{\widetilde{P}}(W ; Y)-\widetilde{R}_{1+s}^{\prime}\left(\widetilde{P}_{W \mid X} \widetilde{P}_{X}, P_{Z \mid X}, Q_{Z}\right)\right\},
\end{aligned}
$$

which has a similar form as the conventional secrecy capacity (with secrecy measured by the normalized mutual information $\frac{1}{n} I\left(M ; Z^{n}\right)$ or unnormalized mutual information $\left.I\left(M ; Z^{n}\right)\right)$ given in [3], [4], [30],

$$
C_{\mathrm{MI}}=\max _{P_{W X}}\{I(W ; Y)-I(W ; Z)\} .
$$

Note that $C_{\mathrm{MI}} \geq \max _{Q_{Z}} C_{1+s}\left(Q_{Z}\right)$ for $s \in(0,1]$ and $C_{\mathrm{MI}}=$ $\max _{Q_{Z}} C_{1+s}\left(Q_{Z}\right)$ for $s \in(-1,0]$. This is because our secrecy measure is stronger than the conventional one. Furthermore, when considering the simultaneous transmission of secret and non-secret messages, the optimal rate region [43] [30, Cor. 2] $]^{6}$ is

$$
\mathcal{R}_{\mathrm{MI}}=\bigcup_{\substack{P_{U \mid W} P_{W \mid X} P_{X}: \\
I(U ; Y) \leq I(U ; Z)}}\left\{\begin{array}{l}
\left(R_{0}, R_{1}\right): R_{0}+R_{1} \leq I(W ; Y), \\
R_{1} \leq I(W ; Y \mid U)-I(W ; Z \mid U)
\end{array}\right\},
$$

which is different from the optimal region $\mathcal{R}_{1+s}$ given by us. Obviously $\bigcup_{Q_{Z}} \mathcal{R}_{1+s}\left(Q_{Z}\right) \subseteq \mathcal{R}_{\mathrm{MI}}$. Xu and Chen [43]

${ }^{6}$ Note that here we refer to Corollary 2 of [30], in which the common message rate is set to zero and the $R_{1}$ and $R_{e}$ there respectively correspond to the $R_{0}+R_{1}$ and $R_{1}$ of this paper. Although the setting in Corollary 2 of [30] does not implicitly indicate the secret and non-secret parts, it is easy to show that if divide the total rate into these two parts, the admissible region does not change. 
and Csiszár and Körner [30, Cor. 2] derived the optimal region $\mathcal{R}_{\mathrm{Ml}}$ by using a two-layered code, but for our case, a single-layered code is sufficient to achieve the optimality; a similar conclusion for the $s=0$ case can be drawn from the results in [44]. This is because our secrecy measure requires that $M_{1}$ and $Z^{n}$ are approximately independent (similarly to the conventional setting) but also requires the wiretapper's observation $Z^{n}$ to approximately follow a target memoryless distribution $Q_{Z}^{n}$ (soft-covering the space according to the target distribution). We provide an intuitive interpretation for why a two-layered code is not necessary to achieve the optimal region for our problem. For simplicity, we consider the case with the Rényi parameter equal to 1; If we apply a twolayered code to our setting then to guarantee the soft-covering property (under the TV distance measure, which is weaker than the Rényi divergence), the non-secret message for each layer has to have rates that are appropriately lower bounded as follows: $R_{0}^{(1)}>I(U ; Z), R_{0}^{(1)}+R_{0}^{(2)}>I(U W ; Z)$ for some $P_{U W \mid X}$ and $P_{X} \in \mathcal{P}\left(P_{Z \mid X}, Q_{Z}\right)$ [45], where $R_{0}^{(1)}$ and $R_{0}^{(2)}$ respectively denote the transmission rate of the nonsecret message for the first and second layer. On the other hand, the total rate is still constrained by $I(W ; Y)$, i.e., $R_{0}^{(1)}+R_{0}^{(2)}+R_{1} \leq I(W ; Y)$. Hence the achievable rate pair $\left(R_{0}^{(1)}+R_{0}^{(2)}, R_{1}\right)$ is still in $\mathcal{R}_{1}\left(P_{Z}\right)$. Note that this is true even for the TV distance. As a result, it must also be true for the stronger distance measures such as relative entropy or Rényi divergence.

Remark 27. Both the coding scheme in this paper and that in [30, Cor. 2] require stochastic encoding to achieve the optimal rate regions. The amount of randomness needed to realize the stochastic encoding for the setting similar to that in [30, Cor. 2] was studied in [42], and the case with only an asymptotically vanishing rate of extra randomness available but with nonuniform sources to be transmitted was studied in [28]. For our setting, the admissible rate region for the case with no extra randomness (i.e., the case with deterministic encoders) was provided in Subsection III-A.

Remark 28. The semantic-security capacity $C_{\mathrm{SS}}$ (with the secrecy measure ${ }^{7} \max _{m_{1}} D\left(P_{Z^{n} \mid M_{1}=m_{1}} \| Q_{Z}^{n}\right) \rightarrow 0$ ), studied in [46], is proven to be equal to $C_{\mathrm{Ml}}$. Obviously, this secrecy measure is not weaker than the one considered in this paper (when the Rényi divergence parameter is equal to 1). In fact, by a simple expurgation argument, it is easy to show that the secrecy measure of $D\left(P_{Z^{n} \mid M_{1}} \| Q_{Z}^{n} \mid P_{M_{1}}\right) \rightarrow 0$ implies semantic secrecy (see for example [12, Appendix A] or [11, footnote on p. 6825]. So these two measures are equivalent. In [46] Goldfeld, Cuff, and Permuter focused only on the secrecy capacity $C_{\mathrm{SS}}$, i.e., the maximum transmission rate of the secret message without a constraint on non-secret message required by the legitimate user. Here we consider a more general scenario: the simultaneous transmission of the secret and non-secret messages. By the above-mentioned expurgation argument, we can obtain a complete characterization of the

\footnotetext{
${ }^{7}$ This measure comes from [46, Thm. 2], but is different from and stronger than the original one $\max _{P_{M} \in \mathcal{P}(\mathcal{M})} I\left(M ; Z^{n}\right)$, also considered by Goldfeld, Cuff, and Permuter in [46]. However, both measures result in the same secrecy capacity [46].
}

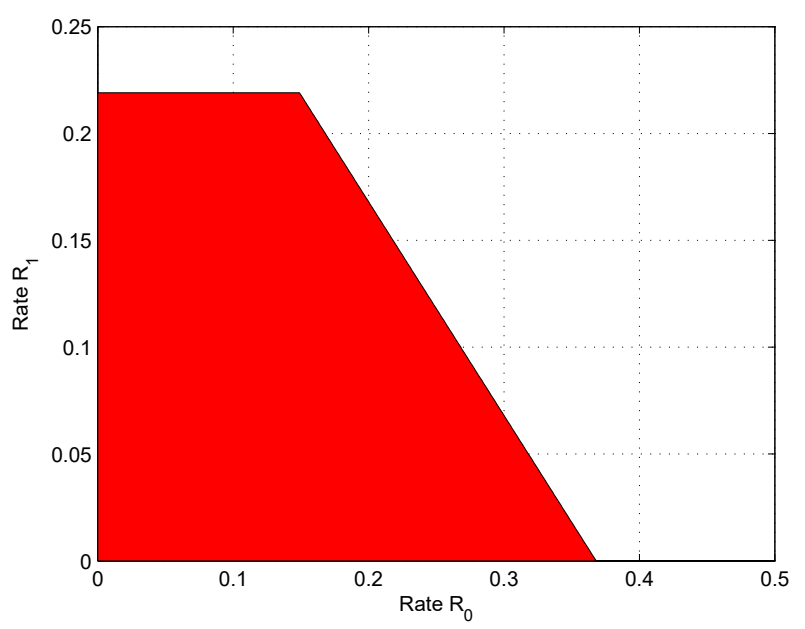

Fig. 6. Illustration of the admissible region for case of using a stochastic encoder and with Rényi parameter $1+s=2$ in (78) or (79) for the binary wiretap channel.

admissible region of $\left(R_{0}, R_{1}\right)$ under the secrecy constraint $\max _{m_{1}} D_{1+s}\left(P_{Z^{n} \mid M_{1}=m_{1}} \| Q_{Z^{n}}\right) \rightarrow 0, s \in[-1,1] \cup\{\infty\}$, which turns out to be the same as $\mathcal{R}_{1+s}\left(Q_{Z}\right)$.

The result of Theorem 6 for the binary wiretap channel $Y=$ $X \oplus V_{1}, V_{1} \sim \operatorname{Bern}(0.1)$ and $Z=X \oplus V_{2}, V_{2} \sim \operatorname{Bern}(0.3)$ with target distribution $Q_{Z}=\operatorname{Bern}(0.5)$ and $s=1$ is illustrated in Fig. 6. From the figure, we observe that different from the deterministic encoder case, for this case the achievability of a rate pair $\left(R_{0}, R_{1}\right)$ indeed implies the achievability of a rate pair $\left(R_{0}^{\prime}, R_{1}^{\prime}\right)$ such that $R_{0}^{\prime} \leq R_{0}, R_{1}^{\prime} \leq R_{1}$.

\section{CONClusion AND Future Work}

In this paper, we studied a generalized version of channel resolvability problem, in which the (normalized or unnormalized) Rényi divergence is used to measure the level of approximation. We also applied these results to the wiretap channel.

Our results generalize or extend several classical and recent results. Our resolvability results extend those by Han and Verdú [2] and by Hayashi [3], [4] as we consider Rényi divergences with orders in $[0,2] \cup\{\infty\}$. Our results for the wiretap channel generalize those by Hou and Kramer [13], and extend those by Wyner [40] and Csiszár and Körner [30], as we measure the effective secrecy (or the leakage) using the Rényi divergence. As discussed in Remark 28, our result on the wiretap channel is also related to the semantic-security capacity studied by Golfeld, Cuff, and Permuter [46].

In the future, we plan to explore various closely related problems to the one contained herein.

1) Rényi common information: Wyner [8] defined the common information between two sources is the minimum rate of commonness needed to simulate these two source in a distributed fashion. In his original work, the normalized relative entropy was used to measure the level of approximation. We can generalize his problem by replacing the relative entropy with the Rényi divergence, 
and define the minimum rate for this case as Rényi common information. In fact, a complete characterization of the Rényi common information for order $\in[0,1]$ and bounds for order $\in(1,2] \cup\{\infty\}$ were provided by us in [16], [20], [21]. Furthermore, the equivalence between the Rényi common information with order $\infty$ and the exact common information was given in [16].

2) Distributed channel synthesis under the Rényi divergence: The coordination problem or distributed channel synthesis problem was studied by Cuff, Permuter, and Cover [9], [47]. In this problem, an observer (encoder) of a source sequence describes the sequence to a distant random number generator (decoder) that produces another sequence. What is the minimum rate of description needed to achieve a joint distribution that is statistically indistinguishable, under the TV distance, from the distribution induced by a given channel? For this problem, Cuff [9] provided a complete characterization of the minimum rate. We can enhance the level of coordination by replacing the TV distance measure with the Rényi divergence. For this enhanced version of the problem, we are interested in characterizing the corresponding admissible rate region. A variant of the infinity-order case was used to study exact channel synthesis by us in [34].

\section{APPENDIX A}

\section{PRELIMINARIES FOR THE PROOFS}

Lemma 3. 1) Assume $\mathcal{X}$ is a finite set. Then for any $P_{X} \in \mathcal{P}(\mathcal{X})$, one can find a sequence of types $T_{X}^{(n)} \in$ $\mathcal{P}^{(n)}(\mathcal{X}), n \in \mathbb{N}$ such that $\left|P_{X}-T_{X}^{(n)}\right| \leq \frac{|\mathcal{X}|}{2 n}$ as $n \rightarrow \infty$.

2) Assume $\mathcal{X}, \mathcal{Y}$ are finite sets. Then for any sequence of types $T_{X}^{(n)} \in \mathcal{P}^{(n)}(\mathcal{X}), n \in \mathbb{N}$ and any $P_{Y \mid X} \in$ $\mathcal{P}(\mathcal{Y} \mid \mathcal{X})$, one can find a sequence of conditional types $V_{Y \mid X}^{(n)} \in \mathcal{P}^{(n)}\left(\mathcal{Y} \mid T_{X}^{(n)}\right), n \in \mathbb{N}$ such that $\mid T_{X}^{(n)} P_{Y \mid X}-$ $T_{X}^{(n)} V_{Y \mid X}^{(n)} \mid \leq \frac{|\mathcal{X}||\mathcal{Y}|}{2 n}$ as $n \rightarrow \infty$.

Statement 1) is exactly [48, Lem. 2.1.2]. The proof of statement 2) follows similarly so its proof is omitted.

We also have the following property concerning the optimization over the set of types and conditional types. To save space, the proof is omitted.

Lemma 4. 1) Assume $\mathcal{X}$ is a finite set. Then for any continuous (under TV distance) function $f: \mathcal{P}(\mathcal{X}) \rightarrow \mathbb{R}$, we have 8

$$
\lim _{n \rightarrow \infty} \min _{P_{X} \in \mathcal{P}^{(n)}(\mathcal{X})} f\left(P_{X}\right)=\min _{P_{X} \in \mathcal{P}(\mathcal{X})} f\left(P_{X}\right)
$$

2) Assume $\mathcal{X}, \mathcal{Y}$ are finite sets. Then for any continuous function $f: \mathcal{P}(\mathcal{X} \times \mathcal{Y}) \rightarrow \mathbb{R}$ and any sequence of types

\footnotetext{
${ }^{8}$ Since $\mathcal{P}(\mathcal{X})$ and $\mathcal{P}^{(n)}(\mathcal{X})$ are compact (closed and bounded) and $f$ is continuous on $\mathcal{P}(\mathcal{X})$, the infima of $\inf _{P_{X} \in \mathcal{P}(\mathcal{X})} f\left(P_{X}\right)$ and $\inf _{P_{X} \in \mathcal{P}^{(n)}(\mathcal{X})} f\left(P_{X}\right)$ are actually minima.
}

$$
\begin{aligned}
T_{X}^{(n)} \in \mathcal{P}^{(n)}(\mathcal{X}), n \in \mathbb{N}, \text { we have } \\
\min _{P_{Y \mid X} \in \mathcal{P}^{(n)}\left(\mathcal{Y} \mid T_{X}^{(n)}\right)} f\left(T_{X}^{(n)} P_{Y \mid X}\right) \\
=\min _{P_{Y \mid X} \in \mathcal{P}(\mathcal{Y} \mid \mathcal{X})} f\left(T_{X}^{(n)} P_{Y \mid X}\right)+o(1) .
\end{aligned}
$$

Remark 29. We have

$$
\begin{aligned}
& \lim _{n \rightarrow \infty} \min _{P_{Y \mid X} \in \mathcal{P}^{(n)}\left(\mathcal{Y} \mid T_{X}^{(n)}\right)} f\left(T_{X}^{(n)} P_{Y \mid X}\right) \\
& =\lim _{n \rightarrow \infty} \min _{P_{Y \mid X} \in \mathcal{P}(\mathcal{Y} \mid \mathcal{X})} f\left(T_{X}^{(n)} P_{Y \mid X}\right)
\end{aligned}
$$

if either one of the limits above exists.

Lemma 5. For any joint type $T_{Y} V_{X \mid Y} \in \mathcal{P}^{(n)}(\mathcal{X} \times \mathcal{Y})$ and any distribution $P_{X^{n}} \in \mathcal{P}\left(\mathcal{X}^{n}\right)$ (not restricted to be i.i.d.), we have

$$
\sum_{y^{n} \in \mathcal{T}_{T_{Y}}} P_{X^{n}}\left(\mathcal{T}_{V_{X \mid Y}}\left(y^{n}\right)\right)=\mathrm{e}^{n H\left(V_{Y \mid X} \mid T_{X}\right)+n \delta_{n}} P_{X^{n}}\left(\mathcal{T}_{T_{X}}\right)
$$

where $T_{X} V_{Y \mid X}=T_{Y} V_{X \mid Y}$.

The proof of Lemma 5 follows from a straightforward application of the method of types [31] and so is omitted.

Lemma 6. [49, Problem 4.15(f)] Assume $\left\{a_{i}\right\}$ are nonnegative real numbers. Then for $p \geq 1$, we have

$$
\sum_{i} a_{i}^{p} \leq\left(\sum_{i} a_{i}\right)^{p}
$$

and for $0<p \leq 1$, we have

$$
\sum_{i} a_{i}^{p} \geq\left(\sum_{i} a_{i}\right)^{p}
$$

Note that $\left(\sum_{i} a_{i}^{p}\right)^{1 / p}$ is a norm for $p \geq 1$, but not for $0<p<1$.

\section{APPENDIX B}

PROOFS OF LEMMAS 1 AND 2

\section{A. Direct Part for Case $1+s$ with $s \in[0,1]$}

Observe that

$$
\begin{aligned}
& \mathrm{e}^{s D_{1+s}\left(P_{Y \mathcal{C}} \| Q_{Y} \times P_{\mathcal{C}}\right)} \\
& =\mathbb{E}_{\mathcal{C}} \sum_{y} P^{1+s}(y \mid \mathcal{C}) Q^{-s}(y) \\
& =\mathbb{E}_{\mathcal{C}} \sum_{y} \sum_{m} P(m) P\left(y \mid f_{\mathcal{C}}(m)\right)\left(P(m) P\left(y \mid f_{\mathcal{C}}(m)\right)\right. \\
& \left.\quad+\sum_{m^{\prime} \neq m} P\left(m^{\prime}\right) P\left(y \mid f_{\mathcal{C}}\left(m^{\prime}\right)\right)\right)^{s} Q^{-s}(y)
\end{aligned}
$$

Then using Lemma 6, we get

$$
\mathrm{e}^{s D_{1+s}\left(P_{Y \mathcal{C}} \| Q_{Y} \times P_{\mathcal{C}}\right)} \leq L_{1}+L_{2}
$$


where

$$
\begin{aligned}
L_{1}:= & \sum_{y} \sum_{m} P^{1+s}(m) \mathbb{E}_{\mathcal{C}}\left[P^{1+s}\left(y \mid f_{\mathcal{C}}(m)\right)\right] Q^{-s}(y) \\
L_{2}:= & \mathbb{E}_{\mathcal{C}} \sum_{y} \sum_{m} P(m) P\left(y \mid f_{\mathcal{C}}(m)\right) \\
& \times\left(\sum_{m^{\prime} \neq m} P\left(m^{\prime}\right) P\left(y \mid f_{\mathcal{C}}\left(m^{\prime}\right)\right)\right)^{s} Q^{-s}(y)
\end{aligned}
$$

Furthermore, $L_{1}$ and $L_{2}$ can be respectively expressed and upper bounded as follows.

$$
\begin{aligned}
L_{1} & =\sum_{y} \sum_{m} P^{1+s}(m) \sum_{x} P(x) P^{1+s}(y \mid x) Q^{-s}(y) \\
& =\mathrm{e}^{\log \sum_{x, y} P(x) P^{1+s}(y \mid x) Q^{-s}(y)-s R},
\end{aligned}
$$

and

$$
\begin{aligned}
L_{2}= & \sum_{y} \sum_{m} P(m) \mathbb{E}_{\mathcal{C}}\left[P\left(y \mid f_{\mathcal{C}}(m)\right)\right] \\
& \times \mathbb{E}_{\mathcal{C}}\left[\left(\sum_{m^{\prime} \neq m} P\left(m^{\prime}\right) P\left(y \mid f_{\mathcal{C}}\left(m^{\prime}\right)\right)\right)^{s}\right] Q^{-s}(y) \\
\leq & \sum_{y} \sum_{m} P(m) \mathbb{E}_{\mathcal{C}}\left[P\left(y \mid f_{\mathcal{C}}(m)\right)\right] \\
& \times\left(\sum_{m^{\prime} \neq m} P\left(m^{\prime}\right) \mathbb{E}_{\mathcal{C}}\left[P\left(y \mid f_{\mathcal{C}}\left(m^{\prime}\right)\right)\right]\right)^{s} Q^{-s}(y) \\
= & \sum_{y} \sum_{m} P(m) \sum_{x} P(x) P(y \mid x) \\
& \times\left(\sum_{m^{\prime} \neq m} P\left(m^{\prime}\right) \sum_{x} P(x) P(y \mid x)\right)^{s} Q^{-s}(y) \\
\leq & \sum_{y} P^{1+s}(y) Q^{-s}(y) \\
= & \mathrm{e}^{s D_{1+s}\left(P_{Y} \| Q_{Y}\right)}
\end{aligned}
$$

where (98) follows since $f_{\mathcal{C}}(m)$ and $f_{\mathcal{C}}\left(m^{\prime}\right)$ are independent for $m \neq m^{\prime}$, and (99) follows since $x \mapsto x^{s}$ is a concave function.

Combining (92), (97) and (102) gives us

$$
\begin{aligned}
& \mathrm{e}^{s D_{1+s}\left(P_{Y \mathcal{C}} \| Q_{Y} \times P_{\mathcal{C}}\right)} \\
& \leq \mathrm{e}^{\log \sum_{x, y} P(x) P^{1+s}(y \mid x) Q^{-s}(y)-s R}+\mathrm{e}^{s D_{1+s}\left(P_{Y} \| Q_{Y}\right)} \\
& \leq 2 \max \left\{\mathrm{e}^{\log \sum_{x, y} P(x) P^{1+s}(y \mid x) Q^{-s}(y)-s R}, \mathrm{e}^{s D_{1+s}\left(P_{Y} \| Q_{Y}\right)}\right\} \\
& =2 \mathrm{e}^{s \Gamma_{1+s}\left(P_{Y \mid X}, Q_{Y}, R\right)} .
\end{aligned}
$$

B. Direct Part for Case $1-s$ with $s \in(0,1)$

For the random code given in Lemma 1, we have

$$
\begin{aligned}
& \mathrm{e}^{-s D_{1-s}\left(P_{Y \mathcal{C}} \| Q_{Y} \times P_{\mathcal{C}}\right)} \\
& =\mathbb{E}_{\mathcal{C}} \sum_{y} P^{1-s}(y \mid \mathcal{C}) Q^{s}(y) \\
& =\mathbb{E}_{\mathcal{C}} \sum_{y} \sum_{m} P(m) P\left(y \mid f_{\mathcal{C}}(m)\right) \\
& \quad \times\left(\sum_{m} P(m) P\left(y \mid f_{\mathcal{C}}(m)\right)\right)^{-s} Q^{s}(y)
\end{aligned}
$$

$=\mathbb{E}_{\mathcal{C}} \sum_{y} \sum_{m} P(m) P\left(y \mid f_{\mathcal{C}}(m)\right)\left(P(m) P\left(y \mid f_{\mathcal{C}}(m)\right)\right.$

$$
\left.+\sum_{m^{\prime} \neq m} P\left(m^{\prime}\right) P\left(y \mid f_{\mathcal{C}}\left(m^{\prime}\right)\right)\right)^{-s} Q^{s}(y)
$$

$\geq \sum_{y} \sum_{m} P(m) \mathbb{E}_{\mathcal{C}}\left[P\left(y \mid f_{\mathcal{C}}(m)\right)\left(P(m) P\left(y \mid f_{\mathcal{C}}(m)\right)\right.\right.$

$\left.\left.+\sum_{m^{\prime} \neq m} P\left(m^{\prime}\right) \mathbb{E}_{\mathcal{C}}\left[P\left(y \mid f_{\mathcal{C}}\left(m^{\prime}\right)\right)\right]\right)^{-s}\right] Q^{s}(y)$

$=\sum_{y} \sum_{m} P(m) \mathbb{E}_{\mathcal{C}}\left[P\left(y \mid f_{\mathcal{C}}(m)\right)\left(P(m) P\left(y \mid f_{\mathcal{C}}(m)\right)\right.\right.$

$\left.\left.+\sum_{m^{\prime} \neq m} P\left(m^{\prime}\right) \sum_{x} P(x) P(y \mid x)\right)^{-s}\right] Q^{s}(y)$

$\geq \sum_{y} \sum_{m} P(m) \mathbb{E}_{\mathcal{C}}\left[P\left(y \mid f_{\mathcal{C}}(m)\right)\left(P(m) P\left(y \mid f_{\mathcal{C}}(m)\right)\right.\right.$

$$
\left.+P(y))^{-s}\right] Q^{s}(y)
$$

$\geq \sum_{y} \sum_{m} P(m) \mathbb{E}_{\mathcal{C}}\left[P\left(y \mid f_{\mathcal{C}}(m)\right)\right.$

$\left.\times\left(2 \max \left\{P(m) P\left(y \mid f_{\mathcal{C}}(m)\right), P(y)\right\}\right)^{-s}\right] Q^{s}(y)$

$$
\begin{aligned}
& =2^{-s} \sum_{y} \sum_{m} P(m) \mathbb{E}_{\mathcal{C}}\left[P ( y | f _ { \mathcal { C } } ( m ) ) \left(P(m)^{-s}\right.\right. \\
& \quad \times P\left(y \mid f_{\mathcal{C}}(m)\right)^{-s} 1\left\{P(m) P\left(y \mid f_{\mathcal{C}}(m)\right) \geq P(y)\right\} \\
& \left.\left.\quad+P^{-s}(y) 1\left\{P(m) P\left(y \mid f_{\mathcal{C}}(m)\right)<P(y)\right\}\right)\right] Q^{s}(y)
\end{aligned}
$$

$$
\begin{aligned}
=2^{-s} & \left(\sum _ { y } \sum _ { m } \mathbb { E } _ { \mathcal { C } } \left[\left(P(m) P\left(y \mid f_{\mathcal{C}}(m)\right)\right)^{1-s}\right.\right. \\
& \left.\times Q^{s}(y) 1\left\{P(m) P\left(y \mid f_{\mathcal{C}}(m)\right) \geq P(y)\right\}\right] \\
& +\sum_{y} \sum_{m} P(m) \mathbb{E}_{\mathcal{C}}\left[P\left(y \mid f_{\mathcal{C}}(m)\right) P^{-s}(y)\right. \\
& \left.\left.\times Q^{s}(y) 1\left\{P(m) P\left(y \mid f_{\mathcal{C}}(m)\right)<P(y)\right\}\right]\right) \\
=2^{-s} & \left(\sum_{m, x, y} \mathrm{e}^{-(1-s) R} P(x) P^{1-s}(y \mid x)\right.
\end{aligned}
$$




$$
\begin{aligned}
& \times Q^{s}(y) 1\left\{\frac{P(y \mid x)}{P(y)} \geq \mathrm{e}^{R}\right\} \\
& +\sum_{m, x, y} P(m) P(x) P(y \mid x) P^{-s}(y) \\
& \left.\times Q^{s}(y) 1\left\{\frac{P(y)}{P(y \mid x)}>\mathrm{e}^{-R}\right\}\right) \\
= & 2^{-s}\left(\mathrm{e}^{s R} \sum_{x, y} P(x) P^{1-s}(y \mid x) Q^{s}(y) 1\left\{\frac{P(y \mid x)}{P(y)} \geq \mathrm{e}^{R}\right\}\right. \\
& \left.+\sum_{x, y} P(x, y) P^{-s}(y) Q^{s}(y) 1\left\{\frac{P(y)}{P(y \mid x)}>\mathrm{e}^{-R}\right\}\right),
\end{aligned}
$$

where (109) follows from that $x \mapsto x^{-s}$ is a convex function and $f_{\mathcal{C}}(m)$ and $f_{\mathcal{C}}\left(m^{\prime}\right)$ are independent for $m \neq m^{\prime}$, and (115) follows since by the construction of the code, $\mathbb{P}\left(f_{\mathcal{C}}(m)=x\right)=P_{X}(x)$.

C. Converse Part for Case $1+s$ with $s \in(0, \infty]$

Observe that

$$
\begin{aligned}
\mathrm{e}^{s D_{1+s}\left(P_{Y} \| Q_{Y}\right)} & \\
= & \sum_{y} P^{1+s}(y) Q^{-s}(y) \\
= & \sum_{y} \sum_{m} P(m) P(y \mid f(m))(P(m) P(y \mid f(m)) \\
& \left.\quad+\sum_{m^{\prime} \neq m} P\left(m^{\prime}\right) P\left(y \mid f\left(m^{\prime}\right)\right)\right)^{s} Q^{-s}(y) \\
\geq & \sum_{y} \sum_{m} P(m) P(y \mid f(m))(P(m) P(y \mid f(m)))^{s} Q^{-s}(y) \\
= & \mathrm{e}^{-s R} \sum_{y} \sum_{m} P(m) P^{1+s}(y \mid f(m)) Q^{-s}(y) \\
= & \mathrm{e}^{-s R} \sum_{y, x, m} P(m) P^{1+s}(y \mid x) Q^{-s}(y) 1\{f(m)=x\} \\
= & \mathrm{e}^{-s R} \sum_{x, y} P(x) P^{1+s}(y \mid x) Q^{-s}(y) \\
= & \mathrm{e}^{\log \sum_{x, y} P(x) P^{1+s}(y \mid x) Q^{-s}(y)-s R},
\end{aligned}
$$

where $P(x):=\sum_{m} P(m) 1\{f(m)=x\}$ and $P(y):=$ $\sum_{x} P(x) P(y \mid x)$ respectively denote the distributions of $X$ and $Y$ induced by the mapping $f$.

On the other hand,

$$
\begin{aligned}
& \mathrm{e}^{s D_{1+s}\left(P_{Y} \| Q_{Y}\right)} \\
& =\sum_{y}\left(\sum_{x} P(x) P(y \mid x)\right)^{1+s} Q^{-s}(y) .
\end{aligned}
$$

Putting (123) and (124) together yields the desired result.
D. Converse Part for Case $1-s$ with $s \in[0,1)$

Observe that

$$
\begin{aligned}
& \mathrm{e}^{-s D_{1-s}\left(P_{Y} \| Q_{Y}\right)} \\
& =\sum_{y} P^{1-s}(y) Q^{s}(y) \\
& =\sum_{y}\left(\sum_{m} P(m) P(y \mid f(m))\right)^{1-s} Q^{s}(y) \\
& =\sum_{y}\left(\sum_{m} P(m) P(y \mid f(m))\right)^{1-s} Q^{s}(y) \\
& \quad \times\left(1\left\{P(m) P(y \mid f(m)) \geq \sum_{m^{\prime} \neq m} P\left(m^{\prime}\right) P\left(y \mid f\left(m^{\prime}\right)\right)\right\}\right. \\
& \left.\quad+1\left\{P(m) P(y \mid f(m))<\sum_{m^{\prime} \neq m} P\left(m^{\prime}\right) P\left(y \mid f\left(m^{\prime}\right)\right)\right\}\right)
\end{aligned}
$$

$$
\begin{aligned}
\leq \sum_{y} & \sum_{m}(P(m) P(y \mid f(m)))^{1-s} Q^{s}(y) \\
\quad \times 1 & \left\{P(m) P(y \mid f(m)) \geq \sum_{m^{\prime} \neq m} P\left(m^{\prime}\right) P\left(y \mid f\left(m^{\prime}\right)\right)\right\} \\
+ & \sum_{y}\left(\sum_{m} P(m) P(y \mid f(m))\right) Q^{1-s}(y) \\
\quad \times 1 & \left\{P(m) P(y \mid f(m))<\sum_{m^{\prime} \neq m} P\left(m^{\prime}\right) P\left(y \mid f\left(m^{\prime}\right)\right)\right\}
\end{aligned}
$$

$$
\begin{aligned}
= & \mathrm{e}^{s R} \sum_{y} \sum_{m} P(m) P^{1-s}(y \mid f(m)) Q^{s}(y) \\
& \times 1\{2 P(m) P(y \mid f(m)) \geq P(y)\} \\
& +\sum_{y}\left(\sum_{m} P(m) P(y \mid f(m))\right)^{1-s} Q^{s}(y) \\
& \quad \times 1\{2 P(m) P(y \mid f(m))<P(y)\} \\
= & \mathrm{e}^{s R} \sum_{y} \sum_{m} P(m) P^{1-s}(y \mid f(m)) Q^{s}(y) \\
& \times 1\left\{\frac{P(y \mid f(m))}{P(y)} \geq \frac{\mathrm{e}^{R}}{2}\right\} \\
+ & \sum_{y} \sum_{m} P(m) P(y \mid f(m)) P(y)^{-s} Q^{s}(y) \\
& \times 1\left\{\frac{P(y \mid f(m))}{P(y)}<\frac{\mathrm{e}^{R}}{2}\right\} \\
\leq & \mathrm{e}^{s R} \sum_{x, y} P(x) P^{1-s}(y \mid x) Q^{s}(y) 1\left\{\frac{P(y \mid x)}{P(y)} \geq \frac{\mathrm{e}^{R}}{2}\right\} \\
& +\sum_{x, y} P(x) P(y \mid x) P^{-s}(y) Q^{s}(y) 1\left\{\frac{P(y \mid x)}{P(y)}<\frac{\mathrm{e}^{R}}{2}\right\}
\end{aligned}
$$

where $P(x):=\sum_{m} P(m) 1\{f(m)=x\}$ and $P(y):=$ $\sum_{x} P(x) P(y \mid x)$ respectively denote the distributions of $X$ and $Y$ induced by the mapping $f$. 
APPENDIX C

PROOF OF PROPOSITION 1

For the $n$-letter version of the problem, $\mathcal{M}_{n}=$ $\left\{1, \ldots, \mathrm{e}^{n R}\right\}$, and the channel $P_{Y \mid X}^{n}$, used $n$ times, can be considered as a superletter channel. Hence the one-shot bounds given in Lemmas 1 and 2 can be used to prove Proposition 1 .

\section{A. Direct Part for Case $1+s$ with $s \in[0,1]$}

By Lemma 1, we have

$$
\begin{aligned}
& \frac{1}{n} D_{1+s}\left(P_{Y^{n} \mathcal{C}_{n}} \| Q_{Y}^{n} \times P_{\mathcal{C}_{n}}\right) \\
\leq & \frac{1}{n} \Gamma_{1+s}\left(P_{X^{n}}, P_{Y^{n} \mid X^{n}}, Q_{Y}^{n}, n R\right)+\frac{1}{n s} \log 2 \\
= & \max \left\{\frac{1}{n} D_{1+s}\left(P_{X^{n} Y^{n}} \| P_{X^{n}} \times Q_{Y}^{n}\right)-R,\right. \\
& \left.\quad \frac{1}{n} D_{1+s}\left(P_{Y^{n}} \| Q_{Y}^{n}\right)\right\}+o(1) .
\end{aligned}
$$

Since $P_{X^{n}}$ is arbitrary, we have

$$
\begin{aligned}
& \frac{1}{n} \inf _{f_{\mathcal{C}_{n}}} D_{1+s}\left(P_{Y^{n} \mathcal{C}_{n}} \| Q_{Y}^{n} \times P_{\mathcal{C}_{n}}\right) \\
& \leq \inf _{P_{X^{n}}} \max \left\{\frac{1}{n} D_{1+s}\left(P_{X^{n} Y^{n}} \| P_{X^{n}} \times Q_{Y}^{n}\right)-R,\right. \\
& \left.\quad \frac{1}{n} D_{1+s}\left(P_{Y^{n}} \| Q_{Y}^{n}\right)\right\}+o(1) \\
& =\Gamma_{1+s}^{(n)}\left(P_{Y \mid X}, Q_{Y}, R\right)+o(1) .
\end{aligned}
$$

B. Converse Part for Case $1+s$ with $s \in(0, \infty]$

By Lemma 2, we have

$$
\begin{aligned}
& \frac{1}{n} D_{1+s}\left(P_{Y^{n} \mathcal{C}_{n}} \| Q_{Y}^{n} \times P_{\mathcal{C}_{n}}\right) \\
\geq & \frac{1}{n} \Gamma_{1+s}\left(P_{X^{n}}, P_{Y^{n} \mid X^{n}}, Q_{Y}^{n}, n R\right) \\
= & \max \left\{\frac{1}{n} D_{1+s}\left(P_{X^{n} Y^{n}} \| P_{X^{n}} \times Q_{Y}^{n}\right)-R,\right. \\
& \left.\frac{1}{n} D_{1+s}\left(P_{Y^{n}} \| Q_{Y}^{n}\right)\right\} \\
\geq & \inf _{P_{X^{n}}} \max \left\{\frac{1}{n} D_{1+s}\left(P_{X^{n} Y^{n}} \| P_{X^{n}} \times Q_{Y}^{n}\right)-R,\right. \\
& \left.\frac{1}{n} D_{1+s}\left(P_{Y^{n}} \| Q_{Y}^{n}\right)\right\} \\
= & \Gamma_{1+s}^{(n)}\left(P_{Y \mid X}, Q_{Y}, R\right) .
\end{aligned}
$$

C. Direct Part for Case $1-s$ with $s \in(0,1)$

Choose $P_{X^{n}}=P_{X}^{n}$ for some $P_{X}$. By Lemma 1, we have

$$
\begin{aligned}
& \mathrm{e}^{-s D_{1-s}\left(P_{Y^{n}} \mathcal{C}_{n} \| Q_{Y}^{n} \times P_{\mathcal{C}_{n}}\right)} \\
& \geq 2^{-s}\left[\mathrm{e}^{n s R} \sum_{x^{n}, y^{n}} P\left(x^{n}\right) P^{1-s}\left(y^{n} \mid x^{n}\right) Q^{s}\left(y^{n}\right)\right. \\
& \times 1\left\{\frac{P\left(y^{n} \mid x^{n}\right)}{P\left(y^{n}\right)} \geq \mathrm{e}^{n R}\right\} \\
& +\sum_{x^{n}, y^{n}} P\left(x^{n}\right) P\left(y^{n} \mid x^{n}\right) P^{-s}\left(y^{n}\right) Q^{s}\left(y^{n}\right) \\
& \left.\times 1\left\{\frac{P\left(y^{n} \mid x^{n}\right)}{P\left(y^{n}\right)}<\mathrm{e}^{n R}\right\}\right] \\
& =2^{-s}\left[\mathrm{e}^{n s R} \Phi_{1}^{n} \sum_{x^{n}, y^{n}} \frac{P\left(x^{n}\right) P^{1-s}\left(y^{n} \mid x^{n}\right) Q^{s}\left(y^{n}\right)}{\Phi_{1}^{n}}\right. \\
& \times 1\left\{\frac{P\left(y^{n} \mid x^{n}\right)}{P\left(y^{n}\right)} \geq \mathrm{e}^{n R}\right\} \\
& +\Phi_{2}^{n} \sum_{x^{n}, y^{n}} \frac{P\left(x^{n}\right) P\left(y^{n} \mid x^{n}\right) P^{-s}\left(y^{n}\right) Q^{s}\left(y^{n}\right)}{\Phi_{2}^{n}} \\
& \left.\times 1\left\{\frac{P\left(y^{n} \mid x^{n}\right)}{P\left(y^{n}\right)}<\mathrm{e}^{n R}\right\}\right],
\end{aligned}
$$

where

$$
\begin{aligned}
\Phi_{1} & :=\left(\sum_{x^{n}, y^{n}} P\left(x^{n}\right) P^{1-s}\left(y^{n} \mid x^{n}\right) Q^{s}\left(y^{n}\right)\right)^{1 / n} \\
& =\mathrm{e}^{-s D_{1-s}\left(P_{X Y} \| P_{X} \times Q_{Y}\right)} \\
\Phi_{2} & :=\left(\sum_{x^{n}, y^{n}} P\left(x^{n}\right) P\left(y^{n} \mid x^{n}\right) P^{-s}\left(y^{n}\right) Q^{s}\left(y^{n}\right)\right)^{1 / n} \\
& =\mathrm{e}^{-s D_{1-s}\left(P_{Y} \| Q_{Y}\right)} .
\end{aligned}
$$

According to large deviation theory [48] (Cramér's theorem), we have

$$
\begin{aligned}
& \lim _{n \rightarrow \infty}-\frac{1}{n} \log \sum_{x^{n}, y^{n}} \frac{P\left(x^{n}\right) P^{1-s}\left(y^{n} \mid x^{n}\right) Q^{s}\left(y^{n}\right)}{\Phi_{1}^{n}} \\
& \times 1\left\{\frac{P\left(y^{n} \mid x^{n}\right)}{P\left(y^{n}\right)} \geq \mathrm{e}^{n R}\right\} \\
&=\max _{t \geq 0}\left(-\log \sum_{x, y} \frac{P(x) P^{1-s}(y \mid x) Q^{s}(y)}{\Phi_{1}}\left(\frac{P(y \mid x)}{P(y) \mathrm{e}^{R}}\right)^{t}\right) \\
&=\max _{t \geq 0}\left(t R-\log \sum_{x, y} P(x) P^{1-(s-t)}(y \mid x) P^{-t}(y) Q^{s}(y)\right) \\
& \quad+\log \Phi_{1},
\end{aligned}
$$


and

$$
\begin{aligned}
& \lim _{n \rightarrow \infty}-\frac{1}{n} \log \sum_{x^{n}, y^{n}} \frac{P\left(x^{n}\right) P\left(y^{n} \mid x^{n}\right) P^{-s}\left(y^{n}\right) Q^{s}\left(y^{n}\right)}{\Phi_{2}^{n}} \\
& \times 1\left\{\frac{P\left(y^{n} \mid x^{n}\right)}{P\left(y^{n}\right)}<\mathrm{e}^{n R}\right\} \\
&=\max _{t \geq 0}\left(-\log \sum_{x, y} \frac{P(x, y) P^{-s}(y) Q^{s}(y)}{\Phi_{2}}\left(\frac{\mathrm{e}^{R} P(y)}{P(y \mid x)}\right)^{t}\right) \\
&=\max _{t \geq 0}\left(-t R-\log \sum_{x, y} P(x) P^{1-t}(y \mid x) P^{t-s}(y) Q^{s}(y)\right) \\
& \quad+\log \Phi_{2} .
\end{aligned}
$$

Substituting these into (141), we have

$\mathrm{e}^{-s D_{1-s}\left(P_{Y}{ }^{n} \mathcal{C}_{n} \| Q_{Y}^{n} \times P_{\mathcal{C}_{n}}\right)}$

$\geq 2^{-s}\left\{\mathrm{e}^{-n \max _{t \geq 0} \tau(R, s, s-t)-n \delta_{n}}+\mathrm{e}^{-n \max _{t \geq 0} \tau(R, s, t)-n \delta_{n}^{\prime}}\right\}$,

where

$$
\begin{aligned}
& \tau(R, s, t) \\
& :=-t R-\log \sum_{x, y} P(x) P^{1-t}(y \mid x) P^{-(s-t)}(y) Q^{s}(y) .
\end{aligned}
$$

That is,

$$
\begin{aligned}
& \frac{1}{n} D_{1-s}\left(P_{Y^{n} \mathcal{C}_{n}} \| Q_{Y}^{n} \times P_{\mathcal{C}_{n}}\right) \\
& \leq \frac{1}{s} \min \left\{\max _{t \geq 0} \tau(R, s, s-t), \max _{t \geq 0} \tau(R, s, t)\right\}+\delta_{n}+\delta_{n}^{\prime} \\
& =\frac{1}{s} \min \left\{\max _{t \leq s} \tau(R, s, t), \max _{t \geq 0} \tau(R, s, t)\right\}+\delta_{n}+\delta_{n}^{\prime}
\end{aligned}
$$

where

$$
\begin{aligned}
& \tau(R, s, t) \\
& :=-t R-\log \sum_{x, y} P(x) P^{1-t}(y \mid x) P^{-(s-t)}(y) Q^{s}(y) .
\end{aligned}
$$

We claim that given $R$ and $s, \tau(R, s, t)$ is concave in $t$; see Lemma 7 below. This implies that

$$
\min \left\{\max _{t \leq s} \tau(R, s, t), \max _{t \geq 0} \tau(R, s, t)\right\}=\max _{t \in[0, s]} \tau(R, s, t) .
$$

Hence we have

$$
\limsup _{n \rightarrow \infty} \frac{1}{n} D_{1-s}\left(P_{Y^{n} \mathcal{C}_{n}} \| Q_{Y}^{n} \times P_{\mathcal{C}_{n}}\right) \leq \frac{1}{s} \max _{t \in[0, s]} \tau(R, s, t) .
$$

Moreover, $P_{X}$ is arbitrary, hence

$$
\begin{aligned}
& \inf _{P_{X^{n}}} \frac{1}{n} D_{1-s}\left(P_{Y^{n} \mathcal{C}_{n}} \| Q_{Y}^{n} \times P_{\mathcal{C}_{n}}\right) \\
& \leq \frac{1}{s} \min _{P_{X}} \max _{t \in[0, s]} \tau(R, s, t)+\delta_{n}+\delta_{n}^{\prime} \\
& =\Gamma_{1-s}^{(1)}\left(P_{Y \mid X}, Q_{Y}, R\right)+\delta_{n}+\delta_{n}^{\prime} .
\end{aligned}
$$

Note that $\Gamma_{1-s}^{(1)}\left(P_{Y \mid X}, Q_{Y}, R\right)$ is a single-letter version of $\Gamma_{1-s}^{(n)}\left(P_{Y \mid X}, Q_{Y}, R\right)$. To achieve the desired result, we set $P_{X^{m k}}=P_{X^{k}}^{m}$ for some fixed $k$. Consider $X^{k}$ as a super-letter, then applying the derivations above, we have as $m \rightarrow \infty$,

$$
\begin{aligned}
& \inf _{P_{X m k}} \frac{1}{m k} D_{1-s}\left(P_{Y^{m k} \mathcal{C}_{m k}} \| Q_{Y}^{m k} \times P_{\mathcal{C}_{m k}}\right) \\
& \leq \Gamma_{1-s}^{(k)}\left(P_{Y \mid X}, Q_{Y}, R\right)+\delta_{m k}+\delta_{m k}^{\prime}
\end{aligned}
$$

where $\delta_{m k}, \delta_{m k}^{\prime} \rightarrow 0$ as $m \rightarrow \infty$ for fixed $k$. When $n$ is not a multiple of $k$, we consider $X^{k}, Y^{k}$ as super-letters, and then apply the code to the first $m:=\left\lfloor\frac{n}{k}\right\rfloor$ super-letters. Then we have

$$
\begin{aligned}
& \inf _{P_{X} n} \frac{1}{n} D_{1-s}\left(P_{Y^{n} \mathcal{C}_{n}} \| Q_{Y}^{n} \times P_{\mathcal{C}_{n}}\right) \\
& \leq \inf _{P_{X^{m k}}} \frac{1}{n} D_{1-s}\left(P_{Y^{m k} \mathcal{C}_{m k}} \| Q_{Y}^{m k} \times P_{\mathcal{C}_{m k}}\right) \\
& \quad+\inf _{P_{X^{l}}} \frac{1}{n} D_{1-s}\left(P_{Y^{l} \mathcal{C}_{l}} \| Q_{Y}^{l} \times P_{\mathcal{C}_{l}}\right) \\
& \leq \inf _{P_{X} m k} \frac{1}{m k} D_{1-s}\left(P_{Y^{m k}} \mathcal{C}_{m k} \| Q_{Y}^{m k} \times P_{\mathcal{C}_{m k}}\right) \\
& \quad+\frac{1}{m} \inf _{P_{X^{l}}} \frac{1}{l} D_{1-s}\left(P_{Y^{l} \mathcal{C}_{l}} \| Q_{Y}^{l} \times P_{\mathcal{C}_{l}}\right),
\end{aligned}
$$

where $l:=n-m k<k$. Observe that

$$
\begin{aligned}
& \inf _{P_{X^{l}}} \frac{1}{l} D_{1-s}\left(P_{Y^{l} \mathcal{C}_{l}} \| Q_{Y}^{l} \times P_{\mathcal{C}_{l}}\right) \\
& \leq \inf _{P_{X}^{l}} \frac{1}{l} D_{1-s}\left(P_{Y^{l} \mathcal{C}_{l}} \| Q_{Y}^{l} \times P_{\mathcal{C}_{l}}\right) \\
& =\inf _{P_{X}} D_{1-s}\left(P_{Y \mathcal{C}_{1}} \| Q_{Y} \times P_{\mathcal{C}_{1}}\right),
\end{aligned}
$$

and the RHS of the inequality above is finite (as assumed in Section I-C). Hence the LHS of the inequality above is also finite. Hence for fixed $k$, we have

$$
\begin{aligned}
& \inf _{P_{X} n} \frac{1}{n} D_{1-s}\left(P_{Y^{n} \mathcal{C}_{n}} \| Q_{Y}^{n} \times P_{\mathcal{C}_{n}}\right) \\
& \leq \inf _{P_{X} m} \frac{1}{m k} D_{1-s}\left(P_{Y^{m k} \mathcal{C}_{m k}} \| Q_{Y}^{m k} \times P_{\mathcal{C}_{m k}}\right)+\delta_{m}^{\prime \prime} \\
& \leq \Gamma_{1-s}^{(k)}\left(P_{Y \mid X}, Q_{Y}, R\right)+\delta_{m k}+\delta_{m k}^{\prime}+\delta_{m}^{\prime \prime} \\
& =\Gamma_{1-s}^{(k)}\left(P_{Y \mid X}, Q_{Y}, R\right)+o(1),
\end{aligned}
$$

where $o(1)$ is a term tending to zero as $m \rightarrow \infty$ or $n \rightarrow \infty$ since $k$ is fixed. Since $k$ is arbitrary, we obtain the desired result.

Lemma 7. Given $R$ and $s, \tau(R, s, t)$ is concave in $t$.

Proof: Define $f(x, y):=P(x) P(y \mid x) P^{-s}(y) Q^{s}(y)$ and $g(x, y):=\frac{P(y)}{P(y \mid x)}$. Then

$$
\begin{aligned}
& -\log \sum_{x, y} P(x) P^{1-t}(y \mid x) P^{-(s-t)}(y) Q^{s}(y) \\
& =-\log \sum_{x, y} f(x, y) g^{t}(x, y) .
\end{aligned}
$$


Assume $t=\lambda t_{1}+(1-\lambda) t_{2}$ for $\lambda \in[0,1]$, then

$$
\begin{aligned}
& \sum_{x, y} f(x, y) g^{t}(x, y) \\
& =\sum_{x, y} f(x, y) g^{\lambda t_{1}+(1-\lambda) t_{2}}(x, y) \\
& =\sum_{x, y}\left(f(x, y) g^{t_{1}}(x, y)\right)^{\lambda}\left(f(x, y) g^{t_{2}}(x, y)\right)^{1-\lambda} \\
& \leq\left(\sum_{x, y} f(x, y) g^{t_{1}}(x, y)\right)^{\lambda}\left(\sum_{x, y} f(x, y) g^{t_{2}}(x, y)\right)^{1-\lambda}
\end{aligned}
$$

where (170) follows from Hölder's inequality.

Hence

$$
\begin{aligned}
& -\log \sum_{x, y} f(x, y) g^{t}(x, y) \\
& \geq-\lambda \log \sum_{x, y} f(x, y) g^{t_{1}}(x, y) \\
& \quad-(1-\lambda) \log \sum_{x, y} f(x, y) g^{t_{2}}(x, y) .
\end{aligned}
$$

That is, $\tau(R, s, t)$ is concave in $t$.

D. Converse Part for Case $1-s$ with $s \in[0,1)$

By Lemma 2, we have for some $P_{X^{n}}$,

$$
\begin{aligned}
& \mathrm{e}^{-s D_{1-s}\left(P_{Y^{n} \mathcal{C}_{n}} \| Q_{Y}^{n} \times P_{\mathcal{C}_{n}}\right)} \\
& \leq \mathrm{e}^{n s R} \sum_{x^{n}, y^{n}} P\left(x^{n}\right) P^{1-s}\left(y^{n} \mid x^{n}\right) Q^{s}\left(y^{n}\right) \\
& \quad \times 1\left\{\frac{P\left(y^{n} \mid x^{n}\right)}{P\left(y^{n}\right)} \geq \frac{\mathrm{e}^{n R}}{2}\right\} \\
& \quad+\sum_{x^{n}, y^{n}} P\left(x^{n}\right) P\left(y^{n} \mid x^{n}\right) P^{-s}\left(y^{n}\right) Q^{s}\left(y^{n}\right) \\
& \quad \times 1\left\{\frac{P\left(y^{n} \mid x^{n}\right)}{P\left(y^{n}\right)}<\frac{\mathrm{e}^{n R}}{2}\right\} .
\end{aligned}
$$

Denote $R^{\prime}:=R-\frac{1}{n} \log 2$. From Markov's inequality, we have

$$
\begin{aligned}
& -\frac{1}{n} \log \sum_{x^{n}, y^{n}} \frac{P\left(x^{n}\right) P^{1-s}\left(y^{n} \mid x^{n}\right) Q^{s}\left(y^{n}\right)}{\Phi_{1}^{n}} \\
& \quad \times 1\left\{\frac{P\left(y^{n} \mid x^{n}\right)}{P\left(y^{n}\right)} \geq \frac{\mathrm{e}^{n R}}{2}\right\} \\
& \geq \max _{t \geq 0}\left(t\left(R-\frac{1}{n} \log 2\right)-\kappa_{1}\right)+\log \Phi_{1} \\
& =\max _{t \geq 0}\left(t R^{\prime}-\kappa_{1}\right)+\log \Phi_{1},
\end{aligned}
$$

and

$$
\begin{aligned}
& -\frac{1}{n} \log \sum_{x^{n}, y^{n}} \frac{P\left(x^{n}\right) P\left(y^{n} \mid x^{n}\right) P^{-s}\left(y^{n}\right) Q^{s}\left(y^{n}\right)}{\Phi_{2}^{n}} \\
& \quad \times 1\left\{\frac{P\left(y^{n} \mid x^{n}\right)}{P\left(y^{n}\right)}<\frac{\mathrm{e}^{n R}}{2}\right\} \\
& \geq \max _{t \geq 0}\left(-t R^{\prime}-\kappa_{2}\right)+\log \Phi_{2}
\end{aligned}
$$

where

$$
\begin{aligned}
& \Phi_{1}:=\left(\sum_{x^{n}, y^{n}} P\left(x^{n}\right) P^{1-s}\left(y^{n} \mid x^{n}\right) Q^{s}\left(y^{n}\right)\right)^{1 / n} \\
& \Phi_{2}:=\left(\sum_{x^{n}, y^{n}} P\left(x^{n}\right) P\left(y^{n} \mid x^{n}\right) P^{-s}\left(y^{n}\right) Q^{s}\left(y^{n}\right)\right)^{1 / n}
\end{aligned}
$$

and

$\kappa_{1}$

$:=\frac{1}{n} \log \sum_{x^{n}, y^{n}} P\left(x^{n}\right) P^{1-s}\left(y^{n} \mid x^{n}\right) Q^{s}\left(y^{n}\right)\left(\frac{P\left(y^{n} \mid x^{n}\right)}{P\left(y^{n}\right)}\right)^{t}$

$=\frac{1}{n} \log \sum_{x^{n}, y^{n}} P\left(x^{n}\right) P^{1-(s-t)}\left(y^{n} \mid x^{n}\right) P^{-t}\left(y^{n}\right) Q^{s}\left(y^{n}\right)$,

$:=\frac{1}{n} \log \sum_{x^{n}, y^{n}} P\left(x^{n}, y^{n}\right) P^{-s}\left(y^{n}\right) Q^{s}\left(y^{n}\right)\left(\frac{P\left(y^{n} \mid x^{n}\right)}{P\left(y^{n}\right)}\right)^{-t}$

$=\frac{1}{n} \log \sum_{x^{n}, y^{n}} P\left(x^{n}\right) P^{1-t}\left(y^{n} \mid x^{n}\right) P^{t-s}\left(y^{n}\right) Q^{s}\left(y^{n}\right)$.

Substituting these into (172), we have

$$
\begin{aligned}
& \mathrm{e}^{-s D_{1-s}\left(P_{Y^{n}} \mathcal{C}_{n} \| Q_{Y}^{n} \times P_{\mathcal{C}_{n}}\right)} \\
& \leq \mathrm{e}^{n\left(s R-\max _{t \geq 0}\left(t R^{\prime}-\kappa_{1}\right)\right)}+\mathrm{e}^{-n \max _{t \geq 0}\left(-t R^{\prime}-\kappa_{2}\right)} \\
& \leq 2 \max \left\{\mathrm{e}^{n\left(s R-\max _{t \geq 0}\left(t R^{\prime}-\kappa_{1}\right)\right)}, \mathrm{e}^{-n \max _{t \geq 0}\left(-t R^{\prime}-\kappa_{2}\right)}\right\} \\
& =2 \mathrm{e}^{-n s \min \left\{\frac{1}{s} \max _{t \geq 0}\left(t R^{\prime}-\kappa_{1}\right)-R, \frac{1}{s} \max _{t \geq 0}\left(-t R^{\prime}-\kappa_{2}\right)\right\}} .
\end{aligned}
$$

That is,

$$
\begin{aligned}
\frac{1}{n} D_{1-s}\left(P_{Y^{n} \mathcal{C}_{n}} \| Q_{Y}^{n} \times P_{\mathcal{C}_{n}}\right) & \\
\geq & \min \left\{\frac{1}{s} \max _{t \geq 0}\left(t R^{\prime}-\kappa_{1}\right)-R^{\prime}-\frac{1}{n} \log 2,\right. \\
& \left.\frac{1}{s} \max _{t \geq 0}\left(-t R^{\prime}-\kappa_{2}\right)\right\}-\delta_{n} \\
\geq & \min \left\{\frac{1}{s} \max _{t \geq 0}\left(t R^{\prime}-\kappa_{1}\right)-R^{\prime}-\frac{1}{n} \log 2,\right. \\
& \left.\frac{1}{s} \max _{t \geq 0}\left(-t R^{\prime}-\kappa_{2}\right)-\frac{1}{n} \log 2\right\}-\delta_{n} \\
= & \min \left\{\frac{1}{s} \max _{t \geq 0}\left(t R^{\prime}-\kappa_{1}\right)-R^{\prime}, \frac{1}{s} \max _{t \geq 0}\left(-t R^{\prime}-\kappa_{2}\right)\right\} \\
& -\delta_{n}-\delta_{n}^{\prime} \\
= & \frac{1}{s} \min \left\{\max _{t \geq 0} \tau\left(R^{\prime}, s, s-t\right), \max _{t \geq 0} \tau\left(R^{\prime}, s, t\right)\right\}-\delta_{n}-\delta_{n}^{\prime} \\
= & \frac{1}{s} \max _{t \in[0, s]} \tau\left(R^{\prime}, s, t\right)-\delta_{n}-\delta_{n}^{\prime}
\end{aligned}
$$




$$
\begin{aligned}
& \geq \frac{1}{s} \max _{t \in[0, s]} \tau(R, s, t)-\delta_{n}-\delta_{n}^{\prime} \\
& \geq \frac{1}{s} \min _{P_{X}} \max _{t \in[0, s]} \tau(R, s, t)-\delta_{n}-\delta_{n}^{\prime} \\
& =\Gamma_{1-s}^{(n)}\left(P_{Y \mid X}, Q_{Y}, R\right)-\delta_{n}-\delta_{n}^{\prime},
\end{aligned}
$$

where the function $\tau(R, s, t)$ is defined in (154), (189) follows from (155), and (190) follows since $\tau(R, s, t)$ is nonincreasing in $R$ for $t \geq 0$.

\section{APPENDIX D \\ Proof of THEOREM 1}

\section{A. Upper Bound for Case $1+s$ with $s \in[0,1]$}

To obtain the upper bound, we set

$$
P\left(x^{n}\right)=\frac{1\left\{x^{n} \in \mathcal{T}_{\widetilde{T}_{X}}\right\}}{\left|\mathcal{T}_{\widetilde{T}_{X}}\right|}
$$

and substitute it into the multiletter expression (32) in Proposition 1 , where $\widetilde{T}_{X}$ is some type of $n$-length sequences. Define $g(x):=\sum_{y} P^{1+s}(y \mid x) Q^{-s}(y)$. Then we obtain

$$
\begin{aligned}
& \frac{1}{n} D_{1+s}\left(P_{X^{n} Y^{n}} \| P_{X^{n}} \times Q_{Y}^{n}\right) \\
& =\frac{1}{n s} \log \sum_{x^{n}} P\left(x^{n}\right) \sum_{y^{n}} P^{1+s}\left(y^{n} \mid x^{n}\right) Q^{-s}\left(y^{n}\right) \\
& =\frac{1}{n s} \log \sum_{x^{n}} P\left(x^{n}\right) \prod_{i=1}^{n} \sum_{y_{i}} P^{1+s}\left(y_{i} \mid x_{i}\right) Q^{-s}\left(y_{i}\right) \\
& =\frac{1}{n s} \log \sum_{x^{n}} P\left(x^{n}\right) \prod_{i=1}^{n} g\left(x_{i}\right) \\
& =\frac{1}{n s} \log \sum_{T_{X}} P_{X^{n}}\left(\mathcal{T}_{T_{X}}\right) \mathrm{e}^{n \sum_{x \in \mathcal{X}} T_{X}(x) \log g(x)} \\
& =\frac{1}{s} \sum_{x} \tilde{T}_{X}(x) \log g(x),
\end{aligned}
$$

where the last line follows from the definition of $P\left(x^{n}\right)$. Furthermore, we also have ${ }^{9}$ (199)-(205), where (203) follows from the fact that both the numbers of $n$-types and $n$-conditional types are polynomial in $n$.

Since $\widetilde{T}_{X}$ is arbitrary, from (198) and (205) we have

$$
\begin{aligned}
& \Gamma_{1+s}^{(n)}\left(P_{Y \mid X}, Q_{Y}, R\right) \\
& \leq \min _{\widetilde{T}_{X}} \max \left\{\frac{1}{s} \sum_{x} \widetilde{T}(x) \log \left(\sum_{y} P^{1+s}(y \mid x) Q^{-s}(y)\right)-R,\right. \\
& \left.\max _{\widetilde{V}_{Y \mid X}} \eta_{1+s}\left(P_{Y \mid X}, Q_{Y}, \widetilde{T}_{X}, \widetilde{V}_{Y \mid X}\right)\right\}+\delta_{n}+\delta_{n}^{\prime} .
\end{aligned}
$$

Note that in (206) the minimization and maximization are taken over the set of types, not the set of general probability

\footnotetext{
${ }^{9}$ As stated in the notation section (Section I-B), for brevity, sometimes we use $T(x, y)$ to denote the joint distributions $T(x) V(y \mid x)$ or $T(y) V(x \mid y)$. Furthermore, for brevity, we use $\sum_{T_{Y}}$ to denote $\sum_{T_{Y} \in \mathcal{P}(n)(\mathcal{Y})}$, and $\sum_{V_{X \mid Y}}$ to denote $\sum_{V_{X \mid Y} \in \mathcal{P}^{(n)}\left(\mathcal{X} \mid T_{Y}\right)}$.
}

mass functions. To achieve the desired result, we continue upper bounding (206) to obtain

$$
\begin{aligned}
& \Gamma_{1+s}^{(n)}\left(P_{Y \mid X}, Q_{Y}, R\right) \\
& \leq \min _{\widetilde{T}_{X}} \max \left\{\frac{1}{s} \sum_{x} \widetilde{T}(x) \log \left(\sum_{y} P^{1+s}(y \mid x) Q^{-s}(y)\right)-R,\right. \\
& \left.\max _{\widetilde{P}_{Y \mid X} \in \mathcal{P}(\mathcal{Y} \mid \mathcal{X})} \eta_{1+s}\left(P_{Y \mid X}, Q_{Y}, \widetilde{T}_{X}, \widetilde{P}_{Y \mid X}\right)\right\}+\delta_{n}+\delta_{n}^{\prime},
\end{aligned}
$$

since $\mathcal{P}^{(n)}\left(\mathcal{Y} \mid \widetilde{T}_{X}\right) \subseteq \mathcal{P}(\mathcal{Y} \mid \mathcal{X})$.

If the objective function of minimization is continuous, then by Lemma 4 we have

$$
\begin{aligned}
& \Gamma_{1+s}^{(n)}\left(P_{Y \mid X}, Q_{Y}, R\right) \\
& \leq \min _{\widetilde{P}_{X} \in \mathcal{P}(\mathcal{X})} \max \left\{\frac{1}{s} \sum_{x} \widetilde{P}(x) \log \left(\sum_{y} P^{1+s}(y \mid x) Q^{-s}(y)\right)\right. \\
& \left.\quad-R, \max _{\widetilde{P}_{Y \mid X} \in \mathcal{P}(\mathcal{Y} \mid \mathcal{X})} \eta_{1+s}\left(P_{Y \mid X}, Q_{Y}, \widetilde{P}_{X}, \widetilde{P}_{Y \mid X}\right)\right\}+o(1) .
\end{aligned}
$$

This completes the proof.

So the rest is to show the continuity of the objective function. To prove this, we only need to show

$$
\max _{\widetilde{P}_{Y \mid X}} \eta_{1+s}\left(P_{Y \mid X}, Q_{Y}, \widetilde{P}_{X}, \widetilde{P}_{Y \mid X}\right)
$$

is continuous in $\widetilde{P}_{X}$. Observe that $\mathcal{P}(\mathcal{Y} \mid \mathcal{X})$ is compact, and $\eta_{1+s}\left(P_{Y \mid X}, Q_{Y}, \widetilde{P}_{X}, \widetilde{P}_{Y \mid X}\right)$ is (jointly) continuous in $\left(\widetilde{P}_{X}, \widetilde{P}_{Y \mid X}\right)$. Hence by the following lemma, we have (209) is continuous in $\widetilde{P}_{X}$.

Lemma 8 (Lemma 14 in [50]). Let $\mathcal{X}$ and $\mathcal{Y}$ be two metric spaces and let $\mathcal{K} \subset \mathcal{X}$ be a compact set. Let $f: \mathcal{X} \times \mathcal{Y} \rightarrow \mathbb{R}$ be a (jointly) continuous real-valued function. Then the function $g: \mathcal{Y} \rightarrow \mathbb{R}$, defined as

$$
g(y):=\min _{x \in \mathcal{K}} f(x, y), \quad \forall y \in \mathcal{Y},
$$

is continuous on $\mathcal{Y}$.

B. Lower Bound for Case $1+s$ with $s \in(0, \infty]$

Observe that (197) still holds. That is,

$$
\begin{aligned}
& \frac{1}{n} D_{1+s}\left(P_{X^{n} Y^{n}} \| P_{X^{n}} \times Q_{Y}^{n}\right) \\
& =\frac{1}{n s} \log \sum_{T_{X}} P_{X^{n}}\left(\mathcal{T}_{T_{X}}\right) \mathrm{e}^{n \sum_{x \in \mathcal{X}} T_{X}(x) \log \sum_{y} P^{1+s}(y \mid x) Q^{-s}(y)} .
\end{aligned}
$$

On the other hand, we also have (216)-(220), where (216) follows from (200), (217) follows from Lemma 6, (218) follows since $x \mapsto x^{1+s}$ is a convex function for $s \geq 0$, and (219) follows from Lemma 5.

Since $\sum_{T_{X}} P_{X^{n}}\left(\mathcal{T}_{T_{X}}\right)=1$ and $\left|\mathcal{P}^{(n)}(\mathcal{X})\right| \leq(n+1)^{|\mathcal{X}|}$, by the pigeonhole principle, we have that there must exist at 


$$
\begin{aligned}
& \frac{1}{n} D_{1+s}\left(P_{Y^{n}} \| Q_{Y}^{n}\right) \\
& =\frac{1}{n s} \log \sum_{y^{n}} P^{1+s}\left(y^{n}\right) Q^{-s}\left(y^{n}\right) \\
& =\frac{1}{n s} \log \left(\sum_{T_{Y}} \sum_{y^{n} \in \mathcal{T}_{T_{Y}}}\left(\sum_{V_{X \mid Y}} \sum_{x^{n} \in \mathcal{T}_{V_{X \mid Y}}\left(y^{n}\right)} P\left(x^{n}\right) \mathrm{e}^{n \sum_{x, y} T(x, y) \log P(y \mid x)}\right)^{1+s} \mathrm{e}^{-n s \sum_{x, y} T(y) \log Q(y)}\right) \\
& =\frac{1}{n s} \log \left(\sum_{T_{Y}} \sum_{y^{n} \in \mathcal{T}_{T_{Y}}}\left(\sum_{V_{X \mid Y}} \sum_{x^{n} \in \mathcal{T}_{V_{X \mid Y}}\left(y^{n}\right)} \frac{1\left\{x^{n} \in \mathcal{T}_{\widetilde{T}_{X}}\right\}}{\left|\mathcal{T}_{\widetilde{T}_{X}}\right|} \mathrm{e}^{n \sum_{x, y} T(x, y) \log P(y \mid x)}\right)^{1+s} \mathrm{e}^{-n s \sum_{y} T(y) \log Q(y)}\right) \\
& =\frac{1}{n s} \log \left(\sum_{T_{Y}} \mathrm{e}^{n H\left(T_{Y}\right)}\left(\sum_{V_{X \mid Y}: V_{X \mid Y} \circ T_{Y}=\widetilde{T}_{X}} \mathrm{e}^{n\left(H\left(V_{X \mid Y} \mid T_{Y}\right)-H\left(\widetilde{T}_{X}\right)+\sum_{x, y} T(x, y) \log P(y \mid x)\right)}\right)^{1+s} \mathrm{e}^{-n s \sum_{y} T(y) \log Q(y)}\right)+\delta_{n} \\
& =\max _{T_{Y}, V_{X \mid Y}: V_{X \mid Y} \circ T_{Y}=\widetilde{T}_{X}} \frac{1}{s}\left(H\left(T_{Y}\right)+(1+s)\left(H\left(V_{X \mid Y} \mid T_{Y}\right)-H\left(\widetilde{T}_{X}\right)+\sum_{x, y} T(x, y) \log P(y \mid x)\right)\right. \\
& \left.-s \sum_{y} T(y) \log Q(y)\right)+\delta_{n}+\delta_{n}^{\prime} \\
& =\max _{T_{Y}, V_{X \mid Y}: V_{X \mid Y} \circ T_{Y}=\widetilde{T}_{X}} \frac{1}{s}\left((1+s) \sum_{x, y} T(x, y) \log \frac{P(y \mid x)}{T(y \mid x)}+s \sum_{y} T(y) \log \frac{T(y)}{Q(y)}\right)+\delta_{n}+\delta_{n}^{\prime} \\
& =\max _{\widetilde{V}_{Y \mid X}}-\frac{1}{s}\left((1+s) \sum_{x, y} \widetilde{T}(x, y) \log \frac{\widetilde{V}(y \mid x)}{P(y \mid x)}-s \sum_{y} \widetilde{T}(y) \log \frac{\widetilde{T}(y)}{Q(y)}\right)+\delta_{n}+\delta_{n}^{\prime},
\end{aligned}
$$

$$
\begin{aligned}
& \frac{1}{n} D_{1+s}\left(P_{Y^{n}} \| Q_{Y}^{n}\right) \\
& =\frac{1}{n s} \log \left(\sum_{T_{Y}} \sum_{y^{n} \in \mathcal{T}_{T_{Y}}}\left(\sum_{V_{X \mid Y}} \sum_{x^{n} \in \mathcal{T}_{V_{X} \mid Y}\left(y^{n}\right)} P\left(x^{n}\right) \mathrm{e}^{n \sum_{x, y} T(x, y) \log P(y \mid x)}\right)^{1+s} \mathrm{e}^{\left.-n s \sum_{x, y} T(y) \log Q(y)\right)}\right. \\
& \geq \frac{1}{n s} \log \left(\sum_{T_{Y}} \sum_{y^{n} \in \mathcal{T}_{T_{Y}}} \sum_{V_{X \mid Y}} P_{X^{n}}^{1+s}\left(\mathcal{T}_{V_{X \mid Y}}\left(y^{n}\right)\right) \mathrm{e}^{n(1+s) \sum_{x, y} T(x, y) \log P(y \mid x)} \mathrm{e}^{\left.-n s \sum_{x, y} T(y) \log Q(y)\right)}\right) \\
& \geq \frac{1}{n s} \log \left(\sum_{T_{Y}, V_{X \mid Y}} \mid \mathcal{T}_{T_{Y} \mid}\left(\sum_{y^{n} \in \mathcal{T}_{T_{Y}}} \frac{1}{\left|\mathcal{T}_{T_{Y} \mid}\right|} P_{X^{n}}\left(\mathcal{T}_{V_{X \mid Y}}\left(y^{n}\right)\right)\right)^{1+s} \mathrm{e}^{n(1+s) \sum_{x, y} T(x, y) \log P(y \mid x)-n s \sum_{x, y} T(y) \log Q(y)}\right) \\
& =\frac{1}{n s} \log \left(\sum_{T_{Y}, V_{X \mid Y}}\left|\mathcal{T}_{T_{Y}}\right|\left(\frac{1}{\mid \mathcal{T}_{T_{Y} \mid}} \mathrm{e}^{n H\left(V_{Y \mid X} \mid T_{X}\right)+n \delta_{n}} P_{X^{n}}\left(\mathcal{T}_{T_{X}}\right)\right)^{1+s} \mathrm{e}^{n(1+s) \sum_{x, y} T(x, y) \log P(y \mid x)-n s \sum_{x, y} T(y) \log Q(y)}\right) \\
& =\frac{1}{n s} \log \left(\sum_{T_{Y}, V_{X \mid Y}} P_{X^{n}}^{1+s}\left(\mathcal{T}_{T_{X}}\right) \mathrm{e}^{n H\left(T_{Y}\right)-n(1+s) I\left(T_{X}, V_{Y \mid X}\right)+n(1+s) \sum_{x, y} T(x, y) \log P(y \mid x)-n s \sum_{x, y} T(y) \log Q(y)+n \delta_{n}+n s \delta_{n}^{\prime}}\right)
\end{aligned}
$$

least one $\widetilde{T}_{X}$ such that $P_{X^{n}}\left(\mathcal{T}_{\widetilde{T}_{X}}\right) \geq(n+1)^{-|\mathcal{X}|}$. Therefore, $\quad=\frac{1}{s} \sum_{x} \widetilde{T}_{X}(x) \log \left(\sum_{y} P^{1+s}(y \mid x) Q^{-s}(y)\right)+\delta_{n}^{\prime \prime}$,
from (211) and (220), we have

$$
\begin{aligned}
& \frac{1}{n} D_{1+s}\left(P_{X^{n} Y^{n}} \| P_{X^{n}} \times Q_{Y}^{n}\right)
\end{aligned}
$$

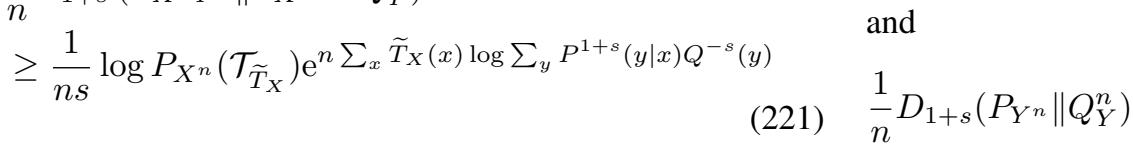




$$
\begin{aligned}
& \geq \frac{1}{n s} \log \left(\sum_{\widetilde{V}_{Y \mid X}} P_{X^{n}}^{1+s}\left(\mathcal{T}_{\widetilde{T}_{X}}\right) \mathrm{e}^{n H\left(\widetilde{T}_{Y}\right)-(1+s) n I\left(\widetilde{T}_{X}, \widetilde{V}_{Y \mid X}\right)}\right. \\
& \left.\times \mathrm{e}^{n(1+s) \sum_{x, y} \widetilde{T}(x, y) \log P(y \mid x)-n s \sum_{x, y} \widetilde{T}(y) \log Q(y)}\right) \\
& +\frac{1}{s} \delta_{n}+\delta_{n}^{\prime} \\
& =\max _{\widetilde{V}_{Y \mid X}} \frac{1}{s}\left(H\left(\widetilde{T}_{Y}\right)+(1+s) I\left(\widetilde{T}_{X}, \widetilde{V}_{Y \mid X}\right)\right. \\
& \left.+(1+s) \sum_{x, y} \widetilde{T}(x, y) \log P(y \mid x)-s \sum_{y} \widetilde{T}(y) \log Q(y)\right) \\
& +\frac{1}{s} \delta_{n}+\delta_{n}^{\prime}+\frac{1+s}{s} \delta_{n}^{\prime \prime} \\
& =\max _{\widetilde{V}_{Y \mid X}}\left(-\frac{1+s}{s} \sum_{x, y} \widetilde{T}(x, y) \log \frac{\widetilde{V}(y \mid x)}{P(y \mid x)}\right. \\
& \left.+\sum_{y} \widetilde{T}(y) \log \frac{\widetilde{T}(y)}{Q(y)}\right)+\frac{1}{s} \delta_{n}+\delta_{n}^{\prime}+\frac{1+s}{s} \delta_{n}^{\prime \prime},
\end{aligned}
$$

where (224) follows from the fact that the number of $n$ conditional types is polynomial in $n$. Therefore,

$$
\begin{aligned}
& \Gamma_{1+s}^{(n)}\left(P_{Y \mid X}, Q_{Y}, R\right) \\
& \geq \min _{\widetilde{T}_{X}} \max \left\{\frac{1}{s} \sum_{x} \widetilde{T}(x) \log \left(\sum_{y} P^{1+s}(y \mid x) Q^{-s}(y)\right)-R,\right. \\
& \left.\quad \max _{\widetilde{V}_{Y \mid X}} \eta_{1+s}\left(P_{Y \mid X}, Q_{Y}, \widetilde{T}_{X}, \widetilde{V}_{Y \mid X}\right)\right\}+o(1) \\
& =\min _{\widetilde{P}_{X}} \max \left\{\frac{1}{s} \sum_{x} \widetilde{P}(x) \log \left(\sum_{y} P^{1+s}(y \mid x) Q^{-s}(y)\right)-R,\right. \\
& \left.\max _{\widetilde{P}_{Y \mid X} \in \mathcal{P}(\mathcal{Y} \mid \mathcal{X})} \eta_{1+s}\left(P_{Y \mid X}, Q_{Y}, \widetilde{P}_{X}, \widetilde{P}_{Y \mid X}\right)\right\}+o(1),
\end{aligned}
$$

where (227) follows from Lemma 4.

\section{Upper Bound for Case $1-s$ with $s \in(0,1)$}

Same as the $1+s$ case, we set $P\left(x^{n}\right)$ as in (193) and substitute it into the multiletter expression (35) in Proposition 1 , where $\widetilde{T}_{X}$ is some type of $n$-length sequences. Then we obtain

$$
\begin{aligned}
- & \frac{1}{n s} \log \sum_{x^{n}, y^{n}} P\left(x^{n}\right) P^{1-t}\left(y^{n} \mid x^{n}\right) P^{t-s}\left(y^{n}\right) Q^{s}\left(y^{n}\right) \\
= & -\frac{1}{n s} \log \sum_{T_{Y} V_{X \mid Y}} \sum_{y^{n} \in \mathcal{T}_{T_{Y}}} \sum_{x^{n} \in \mathcal{T}_{V_{X \mid Y}}\left(y^{n}\right)} P\left(x^{n}\right) P^{1-t}\left(y^{n} \mid x^{n}\right) \\
& \left(\sum_{V_{X \mid Y}} \sum_{x^{n} \in \mathcal{T}_{V_{X \mid Y}}\left(y^{n}\right)} P\left(x^{n}\right) P\left(y^{n} \mid x^{n}\right)\right)^{t-s} Q^{s}\left(y^{n}\right) \\
= & -\frac{1}{n s} \log \sum_{T_{Y} V_{X \mid Y}} \sum_{y^{n} \in \mathcal{T}_{T_{Y}}} \sum_{x^{n} \in \mathcal{T}_{V_{X \mid Y}}\left(y^{n}\right)} P\left(x^{n}\right) \\
& \left(\sum_{V_{X \mid Y}} \sum_{x^{n} \in \mathcal{T}_{V_{X \mid Y}}\left(y^{n}\right)} P\left(x^{n}\right) \mathrm{e}^{n \sum_{x, y} T(x, y) \log P(y \mid x)}\right)^{t-s}
\end{aligned}
$$

$$
\begin{aligned}
& \mathrm{e}^{n(1-t) \sum_{x, y} T(x, y) \log P(y \mid x)+n s \sum_{y} T(y) \log Q(y)} \\
=- & \frac{1}{n s} \log \sum_{T_{Y} V_{X \mid Y}} \sum_{y^{n} \in \mathcal{T}_{T_{Y}}} P_{X^{n}}\left(\mathcal{T}_{V_{X \mid Y}}\left(y^{n}\right)\right) \\
& \left(\sum_{V_{X \mid Y}} P_{X^{n}}\left(\mathcal{T}_{V_{X \mid Y}}\left(y^{n}\right)\right) \mathrm{e}^{n \sum_{x, y} T(x, y) \log P(y \mid x)}\right)^{t-s} \\
& \mathrm{e}^{n(1-t) \sum_{x, y} T(x, y) \log P(y \mid x)+n s \sum_{y} T(y) \log Q(y)} \cdot
\end{aligned}
$$

Observe that for any $y^{n}$ with type $T_{Y}$, we have

$$
\begin{aligned}
& P_{X^{n}}\left(\mathcal{T}_{V_{X \mid Y}}\left(y^{n}\right)\right) \\
& =\mathrm{e}^{-n I\left(V_{X \mid Y}, T_{Y}\right)+n \delta_{n}} 1\left\{V_{X \mid Y} \circ T_{Y}=\widetilde{T}_{X}\right\} .
\end{aligned}
$$

Therefore, we have (232)-(235), where (203) follows from the fact that the number of $n$-types $T_{Y} V_{X \mid Y}$ is polynomial in $n$. Since $\widetilde{T}_{X}$ is arbitrary, by Proposition 1 and (235), we have (236)-(240), where (237) follows since for any function $f(x, y), \max _{x} \min _{y} f(x, y) \leq \min _{y} \max _{x} f(x, y)$, and (239) follows from $\mathcal{P}^{(n)}(\mathcal{Y} \mid \mathcal{X}) \subseteq \mathcal{P}(\mathcal{Y} \mid \mathcal{X})$, Lemma 4 , and the continuity of the objective function of $\min _{\widetilde{P}_{X Y} \in \mathcal{P}(\mathcal{X} \times \mathcal{Y})}$ (the continuity can be shown by Lemma 8).

\section{Lower Bound for Case $1-s$ with $s \in[0,1)$}

Observe that (241)-(248) hold, where (241) follows from (230), (243) follows from that $x \mapsto x^{1+t-s}$ with $0 \leq t \leq s<$ 1 is a concave function, (244) follows from Lemma 5 and the fact $P_{X^{n}}\left(\mathcal{T}_{T_{X}}\right) \leq 1$, and (246) follows from the fact that the number of $n$-types $T_{Y} V_{X \mid Y}$ is polynomial in $n$.

Therefore, from Proposition 1 we have

$$
\begin{aligned}
& \Gamma_{1-s}^{(n)}\left(P_{Y \mid X}, Q_{Y}, R\right) \\
& \geq \min _{P_{X}{ }^{n}} \max _{t \in[0, s]} \min _{T_{X}, V_{Y \mid X}}-\frac{t}{s} R+\frac{t}{s} I\left(V_{Y \mid X}, T_{X}\right) \\
& \quad+\left(\frac{1}{s}-1\right) \sum_{x, y} T(x, y) \log \frac{V(y \mid x)}{P(y \mid x)} \\
& \quad+\sum_{y} T(y) \log \frac{T(y)}{Q(y)}+o(1) \\
&=\max _{t \in[0, s]} \min _{T_{X}, V_{Y \mid X}}-\frac{t}{s} R+\frac{t}{s} I\left(V_{Y \mid X}, T_{X}\right) \\
& \quad+\left(\frac{1}{s}-1\right) \sum_{x, y} T(x, y) \log \frac{V(y \mid x)}{P(y \mid x)} \\
& \quad+\sum_{y} T(y) \log \frac{T(y)}{Q(y)}+o(1) \\
& \geq \max _{t \in[0, s] \widetilde{P}_{X Y} \in \mathcal{P}(\mathcal{X} \times \mathcal{Y})}-\frac{t}{s} R+\frac{t}{s} I\left(\widetilde{P}_{Y \mid X}, \widetilde{P}_{X}\right) \\
& \quad+\left(\frac{1}{s}-1\right) \sum_{x, y} \widetilde{P}(x, y) \log \frac{\widetilde{P}(y \mid x)}{P(y \mid x)} \\
& \quad+\sum_{y} \widetilde{P}(y) \log \frac{\widetilde{P}(y)}{Q(y)}+o(1) \\
&=\min _{\widetilde{P}_{X Y} \in \mathcal{P}(\mathcal{X} \times \mathcal{Y})} \max _{t \in[0, s]}-\frac{t}{s} R+\frac{t}{s} I\left(\widetilde{P}_{Y \mid X}, \widetilde{P}_{X}\right)
\end{aligned}
$$




$$
\begin{aligned}
& -\frac{1}{n s} \log \sum_{x^{n}, y^{n}} P\left(x^{n}\right) P^{1-t}\left(y^{n} \mid x^{n}\right) P^{t-s}\left(y^{n}\right) Q^{s}\left(y^{n}\right) \\
& \leq-\frac{1}{n s} \log \sum_{T_{Y}} \sum_{y^{n} \in \mathcal{T}_{T_{Y}}} \sum_{V_{X \mid Y}} \mathrm{e}^{-n I\left(V_{X \mid Y}, T_{Y}\right)+n \delta_{n}} 1\left\{V_{X \mid Y} \circ T_{Y}=\widetilde{T}_{X}\right\} \\
& \times \mathrm{e}^{n(1-t) \sum_{x, y} T(x, y) \log P(y \mid x)+n s \sum_{y} T(y) \log Q(y)} \\
& \times\left(\sum_{V_{X \mid Y}} \mathrm{e}^{-n I\left(V_{X \mid Y}, T_{Y}\right)+n \delta_{n}} 1\left\{V_{X \mid Y} \circ T_{Y}=\widetilde{T}_{X}\right\} \mathrm{e}^{n \sum_{x, y} T(x, y) \log P(y \mid x)}\right)^{t-s} \\
& =-\frac{1}{n s} \log \max _{T_{Y}, V_{X \mid Y}: V_{X \mid Y} \circ T_{Y}=\widetilde{T}_{X}}\left\{\mathrm{e}^{n H\left(T_{Y}\right)-n I\left(V_{X \mid Y}, T_{Y}\right)+n(1-t) \sum_{x, y} T(x, y) \log P(y \mid x)+n s \sum_{y} T(y) \log Q(y)}\right. \\
& \left.\times\left(\max _{V_{X \mid Y}: V_{X \mid Y} \circ T_{Y}=\widetilde{T}_{X}} \mathrm{e}^{-n I\left(V_{X \mid Y} T_{Y}\right)} \mathrm{e}^{n \sum_{x, y} T(y) V(x \mid y) \log P(y \mid x)}\right)^{t-s}\right\}+(1+t-s) \delta_{n}+\delta_{n}^{\prime} \\
& =-\frac{1}{s} \max _{\widetilde{V}_{Y \mid X}}\left\{H\left(\widetilde{T}_{Y}\right)-I\left(\widetilde{V}_{Y \mid X}, \widetilde{T}_{X}\right)+(1-t) \sum_{x, y} \widetilde{T}(x, y) \log P(y \mid x)+s \sum_{y} \widetilde{T}(y) \log Q(y)\right. \\
& \left.+(t-s)\left(\max _{\widehat{V}_{Y \mid X}: \widehat{V}_{Y \mid X} \circ \widetilde{T}_{X}=\widetilde{V}_{Y \mid X} \circ \widetilde{T}_{X}}-H\left(\widetilde{V}_{Y \mid X} \circ \widetilde{T}_{X}\right)-\sum_{x, y} \widetilde{T}(x) \widehat{V}(y \mid x) \log \frac{\widehat{V}(y \mid x)}{P(y \mid x)}\right)\right\}+(1+t-s) \delta_{n}+\delta_{n}^{\prime} \\
& =-\frac{1}{s} \max _{\widetilde{V}_{Y \mid X}}\left\{-\sum_{x, y} \widetilde{T}(x, y) \log \frac{\widetilde{V}(y \mid x)}{P(y \mid x)}+t \sum_{x, y} \widetilde{T}(x, y) \log \frac{\widetilde{T}(y)}{P(y \mid x)}-s \sum_{y} \widetilde{T}(y) \log \frac{\widetilde{T}(y)}{Q(y)}\right. \\
& \left.+(s-t) \min _{\widehat{V}_{Y \mid X}: \widehat{V}_{Y \mid X} \circ \widetilde{T}_{X}=\widetilde{V}_{Y \mid X} \circ \widetilde{T}_{X}} \sum_{x, y} \widetilde{T}(x) \widehat{V}(y \mid x) \log \frac{\widehat{V}(y \mid x)}{P(y \mid x)}\right\}+(1+t-s) \delta_{n}+\delta_{n}^{\prime},
\end{aligned}
$$

$$
\begin{aligned}
& \quad+\left(\frac{1}{s}-1\right) \sum_{x, y} \widetilde{P}(x, y) \log \frac{\widetilde{P}(y \mid x)}{P(y \mid x)} \\
& \quad+\sum_{y} \widetilde{P}(y) \log \frac{\widetilde{P}(y)}{Q(y)}+o(1) \\
& =\min _{\widetilde{P}_{X Y} \in \mathcal{P}(\mathcal{X} \times \mathcal{Y})} \max \left\{\left(\frac{1}{s}-1\right) \sum_{x, y} \widetilde{P}(x, y) \log \frac{\widetilde{P}(y \mid x)}{P(y \mid x)}\right. \\
& \quad+\sum_{x, y} \widetilde{P}(x, y) \log \frac{\widetilde{P}(y \mid x)}{Q(y)}-R \\
& \quad\left(\frac{1}{s}-1\right) \sum_{x, y} \widetilde{P}(x, y) \log \frac{\widetilde{P}(y \mid x)}{P(y \mid x)} \\
& \left.\quad+\sum_{x, y} \widetilde{P}(y) \log \frac{\widetilde{P}(y)}{Q(y)}\right\}+o(1) \\
& =\Gamma_{1-s}^{\mathrm{LB}}\left(P_{Y \mid X}, Q_{Y}, R\right)+o(1)
\end{aligned}
$$

where the swapping of min and max in (252) follows from the fact that the objective function, equal to

$$
\begin{aligned}
-\frac{t}{s} R & +\frac{t}{s} \sum_{x, y} \widetilde{P}(x, y) \log \frac{\widetilde{P}(y \mid x)}{\widetilde{P}(y)} \\
& +\left(\frac{1}{s}-1\right) \sum_{x, y} \widetilde{P}(x, y) \log \frac{\widetilde{P}(y \mid x)}{P(y \mid x)}
\end{aligned}
$$

$$
\begin{aligned}
& +\sum_{y} \widetilde{P}(y) \log \frac{\widetilde{P}(y)}{Q(y)} \\
=- & \frac{t}{s} R+\left(\frac{1+t}{s}-1\right) \sum_{x, y} \widetilde{P}(x, y) \log \widetilde{P}(y \mid x) \\
& +\left(1-\frac{t}{s}\right) \sum_{y} \widetilde{P}(y) \log \widetilde{P}(y) \\
& -\left(\frac{1}{s}-1\right) \sum_{x, y} \widetilde{P}(x, y) \log P(y \mid x) \\
& -\sum_{y} \widetilde{P}(y) \log Q(y),
\end{aligned}
$$

is convex and concave in $\widetilde{P}_{X Y}$ and $t$ respectively, $\widetilde{P}_{X Y}$ resides in a compact, convex set (the probability simplex) and $t$ resides in a convex set $[0, s]$ (Sion's minimax theorem [51]).

\section{APPENDIX E}

\section{PROOF OF THEOREM 2}

Since the unnormalized Rényi resolvability is not smaller than normalized one, we only need prove the converse part for normalized case and the achievability part for unnormalized case.

A. Converse for Normalized Case with $1+s, s \in(0, \infty]$

We first consider the case $s \in(0,1]$. By Theorem 1, $\lim _{n \rightarrow \infty} \frac{1}{n} \inf _{f_{\mathcal{C}_{n}}} D_{1+s}\left(P_{Y^{n} \mathcal{C}_{n}} \| Q_{Y}^{n} P_{\mathcal{C}_{n}}\right)=0$ if and only if 


$$
\begin{aligned}
& \Gamma_{1-s}^{(n)}\left(P_{Y \mid X}, Q_{Y}, R\right) \\
& \leq \min _{\widetilde{T}_{X}} \max _{t \in[0, s]} \min _{\widetilde{V}_{Y \mid X}}\left\{-\frac{t}{s} R+\frac{1}{s} \sum_{x, y} \widetilde{T}(x, y) \log \frac{\widetilde{V}(y \mid x)}{P(y \mid x)}-\frac{t}{s} \sum_{x, y} \widetilde{T}(x, y) \log \frac{\widetilde{T}(y)}{P(y \mid x)}+\sum_{y} \widetilde{T}(y) \log \frac{\widetilde{T}(y)}{Q(y)}\right. \\
& \left.-\left(1-\frac{t}{s}\right)_{\widehat{V}_{Y \mid X}: \widehat{V}_{Y \mid X} \circ \widetilde{T}_{X}=\widetilde{V}_{Y \mid X} \circ \widetilde{T}_{X}} \sum_{x, y} \widetilde{T}(x) \widehat{V}(y \mid x) \log \frac{\widehat{V}(y \mid x)}{P(y \mid x)}\right\}+o(1) \\
& \leq \min _{\widetilde{T}_{X}} \min _{\widetilde{V}_{Y \mid X}} \max _{t \in[0, s]}\left\{-\frac{t}{s} R+\frac{1}{s} \sum_{x, y} \widetilde{T}(x, y) \log \frac{\widetilde{V}(y \mid x)}{P(y \mid x)}-\frac{t}{s} \sum_{x, y} \widetilde{T}(x, y) \log \frac{\widetilde{T}(y)}{P(y \mid x)}+\sum_{y} \widetilde{T}(y) \log \frac{\widetilde{T}(y)}{Q(y)}\right. \\
& \left.-\left(1-\frac{t}{s}\right)_{\widehat{V}_{Y \mid X}: \widehat{V}_{Y \mid X} \circ \widetilde{T}_{X}=\widetilde{V}_{Y \mid X} \circ \widetilde{T}_{X}} \sum_{x, y} \widetilde{T}(x) \widehat{V}(y \mid x) \log \frac{\widehat{V}(y \mid x)}{P(y \mid x)}\right\}+o(1) \\
& =\min _{\widetilde{T}_{X}} \min _{\widetilde{V}_{Y \mid X}} \max \left\{-R+\sum_{x, y} \widetilde{T}(x, y) \log \frac{P(y \mid x)}{Q(y)}+\frac{1}{s} \sum_{x, y} \widetilde{T}(x, y) \log \frac{\widetilde{V}(y \mid x)}{P(y \mid x)},\right. \\
& \left.\frac{1}{s} \sum_{x, y} \widetilde{T}(x, y) \log \frac{\widetilde{V}(y \mid x)}{P(y \mid x)}+\sum_{y} \widetilde{T}(y) \log \frac{\widetilde{T}(y)}{Q(y)}-\widetilde{V}_{Y \mid X}^{\prime}: \widetilde{V}_{Y \mid X}^{\prime} \min _{\widetilde{T}_{X}=\widetilde{V}_{Y \mid X} \circ \widetilde{T}_{X}} \sum_{x, y} \widetilde{T}(x) \widetilde{V}^{\prime}(y \mid x) \log \frac{\widetilde{V}^{\prime}(y \mid x)}{P(y \mid x)}\right\}+o(1) \\
& \leq \min _{\widetilde{P}_{X Y} \in \mathcal{P}(\mathcal{X} \times \mathcal{Y})} \max \left\{\left(\frac{1}{s}-1\right) \sum_{x, y} \widetilde{P}(x, y) \log \frac{\widetilde{P}(y \mid x)}{P(y \mid x)}+\sum_{x, y} \widetilde{P}(x, y) \log \frac{\widetilde{P}(y \mid x)}{Q(y)}-R,\right. \\
& \frac{1}{s} \sum_{x, y} \widetilde{P}(x, y) \log \frac{\widetilde{P}(y \mid x)}{P(y \mid x)}+\sum_{x, y} \widetilde{P}(y) \log \frac{\widetilde{P}(y)}{Q(y)} \\
& \left.\operatorname{lin}_{\widehat{P}_{Y \mid X} \in \mathcal{P}(\mathcal{Y} \mid \mathcal{X}): \widehat{P}_{Y \mid X} \circ \widetilde{P}_{X}=\widetilde{P}_{Y \mid X} \circ \widetilde{P}_{X}} \sum_{x, y} \widetilde{P}(x) \widehat{P}(y \mid x) \log \frac{\widehat{P}(y \mid x)}{P(y \mid x)}\right\}+o(1) \\
& =\Gamma_{1-s}^{\mathrm{UB}}\left(P_{Y \mid X}, Q_{Y}, R\right)+o(1) .
\end{aligned}
$$

there exists a $\widetilde{P}_{X}$ such that

$$
\begin{array}{r}
\max _{\widetilde{P}_{Y \mid X}} \eta_{1+s}\left(P_{Y \mid X}, Q_{Y}, \widetilde{P}_{X}, \widetilde{P}_{Y \mid X}\right) \leq 0, \\
\frac{1}{s} \sum_{x} \widetilde{P}(x) \log \left(\sum_{y} P^{1+s}(y \mid x) Q^{-s}(y)\right)-R \leq 0 .
\end{array}
$$

On one hand,

$$
\begin{aligned}
& \max _{\widetilde{P}_{Y \mid X}} \eta_{1+s}\left(P_{Y \mid X}, Q_{Y}, \widetilde{P}_{X}, \widetilde{P}_{Y \mid X}\right) \\
& \geq \sum_{x, y} \widetilde{P}(x) P(y \mid x) \log \frac{\sum_{x} \widetilde{P}(x) P(y \mid x)}{Q(y)} .
\end{aligned}
$$

Therefore, (256) implies

$$
\sum_{x} \widetilde{P}(x) P(y \mid x)=Q(y)
$$

i.e.,

$$
\widetilde{P}_{X} \in \mathcal{P}\left(P_{Y \mid X}, Q_{Y}\right)
$$

On the other hand, if $\widetilde{P}_{X} \in \mathcal{P}\left(P_{Y \mid X}, Q_{Y}\right)$, then

$$
\begin{aligned}
& \max _{\widetilde{P}_{Y \mid X}} \eta_{1+s}\left(P_{Y \mid X}, Q_{Y}, \widetilde{P}_{X}, \widetilde{P}_{Y \mid X}\right) \\
& =\max _{\widetilde{P}_{Y \mid X}}\left\{\left(-\frac{1}{s}-1\right) \sum_{x, y} \widetilde{P}(x, y) \log \frac{\widetilde{P}(y \mid x)}{P(y \mid x)}\right. \\
& \left.\quad+\sum_{x, y} \widetilde{P}(y) \log \frac{\widetilde{P}(y)}{Q(y)}\right\} \\
& \leq \max _{\widetilde{P}_{Y \mid X}}\left\{\left(-\frac{1}{s}-1\right) \sum_{x, y} \widetilde{P}(x, y) \log \frac{\sum_{x} \widetilde{P}(y \mid x) \widetilde{P}(x)}{\sum_{x} P(y \mid x) \widetilde{P}(x)}\right. \\
& \left.\quad+\sum_{x, y} \widetilde{P}(y) \log \frac{\widetilde{P}(y)}{Q(y)}\right\} \\
& =\max _{\widetilde{P}_{Y \mid X}}\left\{-\frac{1}{s} \sum_{x, y} \widetilde{P}(y) \log \frac{\widetilde{P}(y)}{Q(y)}\right\} \\
& \leq 0,
\end{aligned}
$$

where follows from the log-sum inequality [52]. Therefore, (256) is equivalent to (260).

Combining (262) and (260) we have

$\inf \left\{R: \lim _{n \rightarrow \infty} \Gamma_{1+s}^{(n)}\left(P_{Y \mid X}, Q_{Y}, R\right)=0\right\}$ 


$$
\begin{aligned}
& -\frac{1}{n s} \log \sum_{x^{n}, y^{n}} P\left(x^{n}, y^{n}\right) P^{-t}\left(y^{n} \mid x^{n}\right) P^{t-s}\left(y^{n}\right) Q^{s}\left(y^{n}\right) \\
& =-\frac{1}{n s} \log \sum_{T_{Y}} \sum_{y^{n} \in \mathcal{T}_{T_{Y}}} \sum_{V_{X \mid Y}} P_{X^{n}}\left(\mathcal{T}_{V_{X \mid Y}}\left(y^{n}\right)\right) \mathrm{e}^{n(1-t) \sum_{x, y} T(x, y) \log P(y \mid x)+n s \sum_{y} T(y) \log Q(y)} \\
& \times\left(\sum_{V_{X \mid Y}} P_{X^{n}}\left(\mathcal{T}_{V_{X \mid Y}}\left(y^{n}\right)\right) \mathrm{e}^{n \sum_{x, y} T(y) V(x \mid y) \log P(y \mid x)}\right)^{t-s} \\
& \geq-\frac{1}{n s} \log \sum_{T_{Y}} \sum_{y^{n} \in \mathcal{T}_{T_{Y}}} \sum_{V_{X \mid Y}} P_{X^{n}}^{1+t-s}\left(\mathcal{T}_{V_{X \mid Y}}\left(y^{n}\right)\right) \mathrm{e}^{n(1-s) \sum_{x, y} T(x, y) \log P(y \mid x)+n s \sum_{y} T(y) \log Q(y)} \\
& \geq-\frac{1}{n s} \log \sum_{T_{Y}, V_{X \mid Y}}\left|\mathcal{T}_{T_{Y}}\right|\left(\sum_{y^{n} \in \mathcal{T}_{T_{Y}}} \frac{1}{\left|\mathcal{T}_{T_{Y}}\right|} P_{X^{n}}\left(\mathcal{T}_{V_{X \mid Y}}\left(y^{n}\right)\right)\right)^{1+t-s} \mathrm{e}^{n(1-s) \sum_{x, y} T(x, y) \log P(y \mid x)+n s \sum_{y} T(y) \log Q(y)} \\
& \geq-\frac{1}{n s} \log \sum_{T_{Y}, V_{X \mid Y}}\left|\mathcal{T}_{T_{Y}}\right|\left(\frac{\mathrm{e}^{n H\left(V_{Y \mid X} \mid T_{X}\right)+n \delta_{n}}}{\left|\mathcal{T}_{T_{Y}}\right|}\right)^{1+t-s} \mathrm{e}^{n(1-s) \sum_{x, y} T(x, y) \log P(y \mid x)+n s \sum_{y} T(y) \log Q(y)} \\
& \geq-\frac{1}{n s} \log \sum_{T_{Y}, V_{X \mid Y}} \mathrm{e}^{n(s-t) H\left(T_{Y}\right)} \mathrm{e}^{n(1+t-s) H\left(V_{Y \mid X} \mid T_{X}\right)+n(1-s) \sum_{x, y} T(x, y) \log P(y \mid x)+n s \sum_{y} T(y) \log Q(y)} \\
& -\frac{1+t-s}{s} \delta_{n}+\delta_{n}^{\prime} \\
& =-\frac{1}{n s} \log \max _{T_{Y}, V_{X \mid Y}} \mathrm{e}^{n(s-t) H\left(T_{Y}\right)+n(1+t-s) H\left(V_{Y \mid X} \mid T_{X}\right)+n(1-s) \sum_{x, y} T(x, y) \log P(y \mid x)+n s \sum_{y} T(y) \log Q(y)} \\
& -\frac{1+t-s}{s} \delta_{n}+\delta_{n}^{\prime}+\delta_{n}^{\prime \prime} \\
& =\min _{T_{Y}, V_{X \mid Y}}-\frac{1}{s}\left(H\left(T_{Y}\right)-(1+t-s) I\left(V_{Y \mid X}, T_{X}\right)+(1-s) \sum_{x, y} T(x, y) \log P(y \mid x)+s \sum_{y} T(y) \log Q(y)\right) \\
& -\frac{1+t-s}{s} \delta_{n}+\delta_{n}^{\prime}+\delta_{n}^{\prime \prime} \\
& =\min _{T_{X}, V_{Y \mid X}} \frac{t}{s} I\left(V_{Y \mid X}, T_{X}\right)+\left(\frac{1}{s}-1\right) \sum_{x, y} T(x, y) \log \frac{V(y \mid x)}{P(y \mid x)}+\sum_{y} T(y) \log \frac{T(y)}{Q(y)} \\
& -\frac{1+t-s}{s} \delta_{n}+\delta_{n}^{\prime}+\delta_{n}^{\prime \prime}
\end{aligned}
$$

$=\inf _{P_{X} \in \mathcal{P}\left(P_{Y \mid X}, Q_{Y}\right)} \frac{1}{s} \sum_{x} P(x) \log \sum_{y} P^{1+s}(y \mid x) Q^{-s}(y)$.

Now we consider the case $s \in(-1,0]$. That is, we need to prove for $s \in(0,1]$,

$$
\begin{aligned}
& \inf \left\{R: \frac{1}{n} \inf _{f_{\mathcal{C}_{n}}} D_{1-s}\left(P_{Y^{n} \mathcal{C}_{n}} \| Q_{Y}^{n} P_{\mathcal{C}_{n}}\right) \rightarrow 0\right\} \\
& \geq \min _{P_{X} \in \mathcal{P}\left(P_{Y \mid X}, Q_{Y}\right)} I(X ; Y) .
\end{aligned}
$$

$$
\left(\frac{1}{s}-1\right) \sum_{x, y} \widetilde{P}(x, y) \log \frac{\widetilde{P}(y \mid x)}{P(y \mid x)}+\sum_{x, y} \widetilde{P}(y) \log \frac{\widetilde{P}(y)}{Q(y)} \leq 0,
$$

$$
\begin{aligned}
\left(\frac{1}{s}-1\right) \sum_{x, y} \widetilde{P}(x, y) \log \frac{\widetilde{P}(y \mid x)}{P(y \mid x)} & \\
& +\sum_{x, y} \widetilde{P}(x, y) \log \frac{\widetilde{P}(y \mid x)}{Q(y)}-R \leq 0 .
\end{aligned}
$$

By Theorem 1, we have

$$
\begin{aligned}
& \inf \left\{R: \Gamma_{1-s}^{\mathrm{LB}}\left(P_{Y \mid X}, Q_{Y}, R\right)=0\right\} \\
& \leq \inf \left\{R: \frac{1}{n} \inf _{f_{\mathcal{C}_{n}}} D_{1-s}\left(P_{Y^{n} \mathcal{C}_{n}} \| Q_{Y^{n}} P_{\mathcal{C}_{n}}\right) \rightarrow 0\right\} .
\end{aligned}
$$

Furthermore, $\Gamma_{1-s}^{\mathrm{LB}}\left(P_{Y \mid X}, Q_{Y}, R\right)=0$ is equivalent to that there exist $\widetilde{P}_{X}, \widetilde{P}_{Y \mid X}$ such that

Note that (268) is equivalent to

$$
\widetilde{P}(y \mid x)=P(y \mid x), \quad \text { and } \quad \widetilde{P}(y)=Q(y) .
$$


Hence (260) also holds. Combining (269) and (260) we have

$$
\begin{aligned}
& \inf \left\{R: \Gamma_{1-s}^{\mathrm{LB}}\left(P_{Y \mid X}, Q_{Y}, R\right)=0\right\} \\
& =\inf _{\widetilde{P}_{X} \in \mathcal{P}\left(P_{Y \mid X}, Q_{Y}\right)} \sum_{x, y} \widetilde{P}(x) P(y \mid x) \log \frac{P(y \mid x)}{Q(y)} \\
& =\inf _{P_{X} \in \mathcal{P}\left(P_{Y \mid X}, Q_{Y}\right)} I(X ; Y) .
\end{aligned}
$$

B. Achievability for Unnormalized Case with $1+s, s \in$ $(-1,1] \cup\{\infty\}$

Next we focus on the achievability part. Since the result for $s \in(-1,0]$ can be obtained from existing works (see Remark 16 ), we only need to prove the case $s \in(0,1] \cup\{\infty\}$.

1) Case $s \in(0,1]$ : We first consider the case $s \in(0,1]$. For this case, by Lemmas 1 and 2, we deduce that

$$
\begin{aligned}
& \inf \left\{R: \inf _{\mathcal{C}_{n}} D_{1+s}\left(P_{Y^{n} \mathcal{C}_{n}} \| Q_{Y}^{n} P_{\mathcal{C}_{n}}\right) \rightarrow 0\right\} \\
& =\inf _{\left\{P_{X^{n}}\right\}: D_{1+s}\left(P_{Y^{n}} \| Q_{Y}^{n}\right) \rightarrow 0} \limsup _{n \rightarrow \infty} \frac{1}{n} D_{1+s}\left(P_{X^{n} Y^{n}} \| P_{X^{n}} Q_{Y}^{n}\right) .
\end{aligned}
$$

Set $P_{X^{n}}\left(x^{n}\right) \propto Q_{X}^{n}\left(x^{n}\right) 1\left\{x^{n} \in \mathcal{T}_{\epsilon}^{n}\left(Q_{X}\right)\right\}$ for some $Q_{X} \in$ $\mathcal{P}\left(P_{Y \mid X}, Q_{Y}\right)$. On one hand,

$$
\begin{aligned}
& D_{1+s}\left(P_{X^{n}} \| Q_{X}^{n}\right) \\
& =\frac{1}{s} \log \sum_{x^{n}}\left(\frac{Q_{X}^{n}\left(x^{n}\right) 1\left\{x^{n} \in \mathcal{T}_{\epsilon}^{n}\right\}}{Q_{X}^{n}\left(\mathcal{T}_{\epsilon}^{n}\right)}\right)^{1+s}\left(Q_{X}^{n}\left(x^{n}\right)\right)^{-s} \\
& =\frac{1}{s} \log \sum_{x^{n} \in \mathcal{T}_{\epsilon}^{n}}\left(\frac{1}{Q_{X}^{n}\left(\mathcal{T}_{\epsilon}^{n}\right)}\right)^{1+s} Q_{X}^{n}\left(x^{n}\right) \\
& =\log \frac{1}{Q_{X}^{n}\left(\mathcal{T}_{\epsilon}^{n}\right)} \\
& \rightarrow 0
\end{aligned}
$$

where (277) follows from the fact that $Q_{X}^{n}\left(\mathcal{T}_{\epsilon}^{n}\right) \rightarrow 1$. By the data processing inequality [35], we have

$$
D_{1+s}\left(P_{Y^{n}} \| Q_{Y}^{n}\right) \leq D_{1+s}\left(P_{X^{n}} \| Q_{X}^{n}\right) .
$$

Hence $D_{1+s}\left(P_{Y^{n}} \| Q_{Y}^{n}\right) \rightarrow 0$ as well.

On the other hand,

$$
\begin{aligned}
& \frac{1}{n} D_{1+s}\left(P_{X^{n} Y^{n}} \| P_{X^{n}} Q_{Y}^{n}\right) \\
& =\frac{1}{n s} \log \sum_{x^{n}} \frac{Q_{X}^{n}\left(x^{n}\right) 1\left\{x^{n} \in \mathcal{T}_{\epsilon}^{n}\right\}}{Q_{X}^{n}\left(\mathcal{T}_{\epsilon}^{n}\right)} \\
& \quad \times \mathrm{e}^{n \sum_{x} T_{x}^{n}(x) \log \sum_{y} P^{1+s}(y \mid x) Q^{-s}(y)} \\
& \leq \frac{1}{n s} \log \sum_{x^{n}} \frac{Q_{X}^{n}\left(x^{n}\right) 1\left\{x^{n} \in \mathcal{T}_{\epsilon}^{n}\right\}}{Q_{X}^{n}\left(\mathcal{T}_{\epsilon}^{n}\right)} \quad \forall x: \mid T_{X}(x)-Q_{X}: \\
& =\quad \mathrm{max}^{n \sum_{x} T_{X}(x) \log \sum_{y} P^{1+s}(y \mid x) Q^{-s}(y)} \\
& \quad \forall x: \mid T_{X}(x)-Q_{X}: \\
& \quad \times \log \sum_{y} P^{1+s}(y \mid x) Q^{-s}(y) \\
& \leq(1+\epsilon) \frac{1}{s} \sum_{x} Q(x) \log \sum_{y} P^{1+s}(y \mid x) Q^{-s}(y) .
\end{aligned}
$$

By letting $n \rightarrow \infty$ and $\epsilon \rightarrow 0$, we have

$$
\begin{aligned}
& \limsup _{n \rightarrow \infty} \frac{1}{n} D_{1+s}\left(P_{X^{n} Y^{n}} \| P_{X^{n}} Q_{Y}^{n}\right) \\
& \leq \frac{1}{s} \sum_{x} Q(x) \log \sum_{y} P^{1+s}(y \mid x) Q^{-s}(y) .
\end{aligned}
$$

Furthermore, since $Q_{X} \in \mathcal{P}\left(P_{Y \mid X}, Q_{Y}\right)$ is arbitrary,

$$
\begin{aligned}
& \limsup _{n \rightarrow \infty} \frac{1}{n} D_{1+s}\left(P_{X^{n} Y^{n}} \| P_{X^{n}} Q_{Y}^{n}\right) \\
& \leq \inf _{Q_{X} \in \mathcal{P}\left(P_{Y \mid X}, Q_{Y}\right)} \frac{1}{s} \sum_{x} Q(x) \log \sum_{y} P^{1+s}(y \mid x) Q^{-s}(y) .
\end{aligned}
$$

Combining this with (273) we have the achievability part for the case of $s \in(0,1]$.

2) Case $s=\infty$ : Let $\epsilon>0$ be such that

$$
R>(1+\epsilon) \sum_{x} Q_{X}(x) D_{\infty}\left(P_{Y \mid X}(\cdot \mid x) \| Q_{Y}\right)+\epsilon .
$$

Here

$Q_{X}:=\underset{P_{X} \in \mathcal{P}\left(P_{Y \mid X}, Q_{Y}\right)}{\arg \min } \sum_{x} P_{X}(x) D_{\infty}\left(P_{Y \mid X}(\cdot \mid x) \| Q_{Y}\right)$.

We set the random code to be $\mathcal{C}_{n}=\left\{X^{n}(m)\right\}_{m \in \mathcal{M}_{n}}$ with $X^{n}(m), m \in \mathcal{M}_{n}$ drawn independently for different $m$ 's and according to the same distribution $P_{X^{n}}$ such that $P_{X^{n}}\left(x^{n}\right) \propto$ $Q_{X}^{n}\left(x^{n}\right) 1\left\{x^{n} \in \mathcal{T}_{\epsilon}^{n}\left(Q_{X}\right)\right\}$. Next we prove that such a sequence of random codes satisfies $\mathbb{E}_{\mathcal{C}_{n}}\left[D_{\infty}\left(P_{Y^{n} \mid \mathcal{C}_{n}} \| Q_{Y}^{n}\right)\right] \rightarrow$ 0 as $n \rightarrow \infty$.

For brevity, in the following we denote $M=\mathrm{e}^{n R}$. According to the definition of the Rényi divergence, we first have

$$
\begin{aligned}
& \mathrm{e}^{\mathbb{E}_{\mathcal{C}_{n}}\left[D_{\infty}\left(P_{Y^{n}} \mid \mathcal{C}_{n} \| Q_{Y}^{n}\right)\right]} \\
& \leq \mathbb{E}_{\mathcal{C}_{n}}\left[\mathrm{e}^{D_{\infty}\left(P_{Y^{n}} \mid \mathcal{C}_{n} \| Q_{Y}^{n}\right)}\right] \\
& =\mathbb{E}_{\mathcal{C}_{n}}\left[\max _{y^{n}} \frac{P_{Y^{n}} \mid \mathcal{C}_{n}\left(y^{n} \mid \mathcal{C}_{n}\right)}{Q_{Y}^{n}\left(y^{n}\right)}\right] \\
& =\mathbb{E}_{\mathcal{C}_{n}}\left[\max _{y^{n}} \widetilde{g}\left(\mathcal{C}_{n}, y^{n}\right)\right],
\end{aligned}
$$

where $\widetilde{g}\left(\mathcal{C}_{n}, y^{n}\right) \quad:=\sum_{m \in \mathcal{M}_{n}} g\left(X^{n}(m), y^{n}\right) / M$ with $g\left(x^{n}, y^{n}\right) \quad:=\quad P_{Y \mid X}^{n}\left(y^{n} \mid x^{n}\right) / Q_{Y}^{n}\left(y^{n}\right)$. Obviously, for any $x^{n} \in \mathcal{T}_{\epsilon}^{n}\left(Q_{X}\right)$, its type $T_{x^{n}}$ satisfies that $\left|T_{x^{n}}(x)-Q_{X}(x)\right| \leq \epsilon Q_{X}(x), \forall x$. Therefore, for any $x^{n} \in \mathcal{T}_{\epsilon}^{n}\left(Q_{X}\right)$ and any $y^{n} \in \mathcal{Y}^{n}$, we have

$$
\begin{aligned}
& g\left(x^{n}, y^{n}\right) \\
& =\mathrm{e}^{n \sum_{x, y} T_{x^{n}} y^{n}(x, y) \log \frac{P_{Y \mid X}(y \mid x)}{Q_{Y}(y)}}
\end{aligned}
$$

$\leq \mathrm{e}^{n \max _{T_{X}: \forall x:\left|T_{X}(x)-Q_{X}(x)\right| \leq \epsilon Q_{X}(x)} \sum_{x} T_{X}(x) \max _{y} \log \frac{P_{Y \mid X}(y \mid x)}{Q_{Y}(y)}}$

$\leq \mathrm{e}^{n(1+\epsilon) \sum_{x} Q_{X}(x) D_{\infty}\left(P_{Y \mid X}(\cdot \mid x) \| Q_{Y}\right)}$

$=: A_{n}$. 
Continuing (289), we get for any $\epsilon^{\prime}>0$,

$$
\begin{aligned}
& \mathrm{e}^{\mathbb{E}_{\mathcal{C}_{n}}\left[D_{\infty}\left(P_{Y^{n} \mid \mathcal{C}_{n}} \| Q_{Y}^{n}\right)\right]} \\
& =\mathbb{E}_{\mathcal{C}_{n}}\left[\max _{y^{n}} \widetilde{g}\left(\mathcal{C}_{n}, y^{n}\right) 1\left\{\max _{y^{n}} \widetilde{g}\left(\mathcal{C}_{n}, y^{n}\right) \geq 1+\epsilon^{\prime}\right\}\right] \\
& \quad+\mathbb{E}_{\mathcal{C}_{n}}\left[\max _{y^{n}} \widetilde{g}\left(\mathcal{C}_{n}, y^{n}\right) 1\left\{\max _{y^{n}} \widetilde{g}\left(\mathcal{C}_{n}, y^{n}\right)<1+\epsilon^{\prime}\right\}\right] \\
& \leq \mathbb{E}_{\mathcal{C}_{n}}\left[A_{n} \cdot 1\left\{\max _{y^{n}} \widetilde{g}\left(\mathcal{C}_{n}, y^{n}\right) \geq 1+\epsilon^{\prime}\right\}\right]+1+\epsilon^{\prime} \\
& =A_{n} \mathbb{P}_{\mathcal{C}_{n}}\left(\max _{y^{n}} \widetilde{g}\left(\mathcal{C}_{n}, y^{n}\right) \geq 1+\epsilon^{\prime}\right)+1+\epsilon^{\prime} \\
& \leq A_{n}|\mathcal{Y}|^{n} \max _{y^{n}} \mathbb{P}_{\mathcal{C}_{n}}\left(\widetilde{g}\left(\mathcal{C}_{n}, y^{n}\right) \geq 1+\epsilon^{\prime}\right)+1+\epsilon^{\prime}
\end{aligned}
$$

where (298) follows from the union bound. Obviously, both $A_{n}$ and $|\mathcal{Y}|^{n}$ are only exponentially growing. Therefore, if the probability $\mathbb{P}_{\mathcal{C}_{n}}\left(\widetilde{g}\left(\mathcal{C}_{n}, y^{n}\right) \geq 1+\epsilon^{\prime}\right)$ vanishes doubly exponentially fast, then $\mathbb{E}_{\mathcal{C}_{n}}\left[D_{\infty}\left(P_{Y^{n} \mid \mathcal{C}_{n}} \| Q_{Y}^{n}\right)\right] \rightarrow \log \left(1+\epsilon^{\prime}\right)$ as $n \rightarrow \infty$. To this end, we use Bernstein's inequality [53] to bound the probability uniformly over all $y^{n}$. Observe that $g\left(X^{n}(m), y^{n}\right), m \in \mathcal{M}_{n}$ are i.i.d. random variables with mean

$$
\begin{aligned}
\mu_{\epsilon, n} & :=\mathbb{E}_{X^{n}}\left[g\left(X^{n}, y^{n}\right)\right] \\
& =\sum_{x^{n}} \frac{Q_{X}^{n}\left(x^{n}\right) 1\left\{x^{n} \in \mathcal{T}_{\epsilon}^{n}\left(Q_{X}\right)\right\}}{Q_{X}^{n}\left(\mathcal{T}_{\epsilon}^{n}\left(Q_{X}\right)\right)} \frac{P_{Y \mid X}^{n}\left(y^{n} \mid x^{n}\right)}{Q_{Y}^{n}\left(y^{n}\right)} \\
& \leq \frac{1}{Q_{X}^{n}\left(\mathcal{T}_{\epsilon}^{n}\left(Q_{X}\right)\right)} \\
& \rightarrow 1, \text { as } n \rightarrow \infty
\end{aligned}
$$

and variance

$$
\begin{aligned}
\operatorname{Var}_{X^{n}}\left[g\left(X^{n}, y^{n}\right)\right] & \leq \mathbb{E}_{X^{n}}\left[g\left(X^{n}, y^{n}\right)^{2}\right] \\
& \leq A_{n} \mu_{\epsilon, n}
\end{aligned}
$$

Then we get

$$
\begin{aligned}
& \mathbb{P}_{\mathcal{C}_{n}}\left(\widetilde{g}\left(\mathcal{C}_{n}, y^{n}\right) \geq 1+\epsilon^{\prime}\right) \\
& =\mathbb{P}_{\mathcal{C}_{n}}\left(\sum_{m \in \mathcal{M}_{n}} g\left(X^{n}(m), y^{n}\right)-\mu_{\epsilon, n} M\right. \\
& \left.\geq\left(1+\epsilon^{\prime}-\mu_{\epsilon, n}\right) M\right) \\
& \leq \exp \left(-\frac{\frac{1}{2}\left(1+\epsilon^{\prime}-\mu_{\epsilon, n}\right)^{2} M^{2}}{M A_{n} \mu_{\epsilon, n}+\frac{1}{3}\left(1+\epsilon^{\prime}-\mu_{\epsilon, n}\right) M A_{n}}\right) \\
& \leq \exp \left(-\frac{3\left(1+\epsilon^{\prime}-\mu_{\epsilon, n}\right)^{2} M}{2\left(1+\epsilon^{\prime}+2 \mu_{\epsilon, n}\right) A_{n}}\right) .
\end{aligned}
$$

Since $\mu_{\epsilon, n} \rightarrow 1$ as $n \rightarrow \infty$, we have that for any $\epsilon^{\prime}>0$, there exists a sufficiently large $n_{0}$ such that $\mu_{\epsilon, n} \leq 1+\frac{\epsilon^{\prime}}{2}$ for $n \geq n_{0}$. Hence for $n \geq n_{0}$, (307) is further upper bounded by $\exp \left(-\frac{3 \epsilon^{\prime 2}}{8\left(3+2 \epsilon^{\prime}\right)} \mathrm{e}^{n \epsilon}\right)$, which converges to zero doubly exponentially fast. Therefore, $\mathbb{E}_{\mathcal{C}_{n}}\left[D_{\infty}\left(P_{Y^{n} \mid \mathcal{C}_{n}} \| Q_{Y}^{n}\right)\right] \rightarrow$ $\log \left(1+\epsilon^{\prime}\right)$ as $n \rightarrow \infty$. Since $\epsilon^{\prime}>0$ is arbitrary, $\mathbb{E}_{\mathcal{C}_{n}}\left[D_{\infty}\left(P_{Y^{n} \mid \mathcal{C}_{n}} \| Q_{Y}^{n}\right)\right] \rightarrow 0$ as $n \rightarrow \infty$.
Note that here we have proven that if the code rate

$$
R>\min _{P_{X} \in \mathcal{P}\left(P_{Y \mid X}, Q_{Y}\right)} \sum_{x} P_{X}(x) D_{\infty}\left(P_{Y \mid X}(\cdot \mid x) \| Q_{Y}\right),
$$

then

$$
\mathbb{E}_{\mathcal{C}_{n}}\left[D_{\infty}\left(P_{Y^{n} \mid \mathcal{C}_{n}} \| Q_{Y}^{n}\right)\right] \rightarrow 0 .
$$

The convergence in (309) implies that there exists a sequence of deterministic codebooks $\left\{c_{n}\right\}$ with rate $R$ such that $D_{\infty}\left(P_{Y^{n} \mid \mathcal{C}_{n}=c_{n}} \| Q_{Y}^{n}\right) \rightarrow 0$. If we set the random mapping $\mathcal{C}_{n}^{\prime}$ to be the deterministic codebook/mapping $c_{n}$, i.e., $\mathcal{C}_{n}^{\prime}=c_{n}$, then $D_{\infty}\left(P_{Y^{n} \mathcal{C}_{n}^{\prime}} \| Q_{Y}^{n} P_{\mathcal{C}_{n}^{\prime}}\right)=D_{\infty}\left(P_{Y^{n} \mid \mathcal{C}_{n}=c_{n}} \| Q_{Y}^{n}\right)$. Therefore, we have $D_{\infty}\left(P_{Y^{n} \mathcal{C}_{n}^{\prime}} \| Q_{Y}^{n} P_{\mathcal{C}_{n}^{\prime}}\right) \rightarrow 0$ as desired.

\section{APPENDIX F \\ PROOF OF THEOREM 3}

Achievability: We first consider $s \in(0,1]$ case. Since $P_{X} \in$ $\mathcal{P}\left(P_{Y \mid X}, Q_{Y}\right), D_{1+s}\left(P_{Y} \| Q_{Y}\right)=0$. By Lemma 1, we obtain

$$
\begin{aligned}
& \left.\mathrm{e}^{s D_{1+s}\left(P_{Y} \mathcal{C}_{n}\right.} \| Q_{Y}^{n} \times P_{\mathcal{C}_{n}}\right) \\
& \leq \mathrm{e}^{n \log \sum_{x, y} P(x) P^{1+s}(y \mid x) Q^{-s}(y)-n s R}+\mathrm{e}^{n s D_{1+s}\left(P_{Y} \| Q_{Y}\right)} \\
& =\mathrm{e}^{n \log \sum_{x, y} P(x) P^{1+s}(y \mid x) Q^{-s}(y)-n s R}+1 \\
& \text { Take } \log \mathrm{s} \\
& \quad s D_{1+s}\left(P_{Y^{n} \mathcal{C}_{n}} \| Q_{Y}^{n} \times P_{\mathcal{C}_{n}}\right) \\
& \quad \leq \log \left(\mathrm{e}^{n \log \sum_{x, y} P(x) P^{1+s}(y \mid x) Q^{-s}(y)-n s R}+1\right) \\
& \quad \leq \mathrm{e}^{-n s\left(R-\frac{1}{s} \log \sum_{x, y} P(x) P^{1+s}(y \mid x) Q^{-s}(y)\right)} \\
& \quad=\mathrm{e}^{-n s\left(R-D_{1+s}\left(P_{X Y} \| P_{X} \times Q_{Y}\right)\right)}
\end{aligned}
$$

Hence

$$
\begin{aligned}
& -\frac{1}{n} \log D_{1+s}\left(P_{Y^{n} \mathcal{C}_{n}} \| Q_{Y}^{n} \times P_{\mathcal{C}_{n}}\right) \\
& \geq s\left(R-D_{1+s}\left(P_{X Y} \| P_{X} \times Q_{Y}\right)\right)+\delta_{n} .
\end{aligned}
$$

This implies $D_{1+s}\left(P_{Y^{n}} \mathcal{C}_{n} \| Q_{Y}^{n} \times P_{\mathcal{C}_{n}}\right)$ vanishes at least exponentially fast for $s \in(0,1]$. Now we refine the exponential rate of decay. Denote $t_{1}^{*} \in[s, 1]$ as the maximizer of $\max _{t \in[s, 1]} t\left(R-D_{1+t}\left(P_{X Y} \| P_{X} \times Q_{Y}\right)\right)$. Since (315) holds for any $s \in(0,1]$, we have for $s \in(0,1]$,

$$
\begin{aligned}
& \liminf _{n \rightarrow \infty}-\frac{1}{n} \log D_{1+s}\left(P_{Y^{n} \mathcal{C}_{n}} \| Q_{Y}^{n} \times P_{\mathcal{C}_{n}}\right) \\
& \geq \liminf _{n \rightarrow \infty}-\frac{1}{n} \log D_{1+t_{1}^{*}}\left(P_{Y^{n} \mathcal{C}_{n}} \| Q_{Y}^{n} \times P_{\mathcal{C}_{n}}\right) \\
& \geq t_{1}^{*}\left(R-D_{1+t_{1}^{*}}\left(P_{X Y} \| P_{X} \times Q_{Y}\right)\right) \\
& =\max _{t \in[s, 1]} t\left(R-D_{1+t}\left(P_{X Y} \| P_{X} \times Q_{Y}\right)\right) .
\end{aligned}
$$

As for $s \in(-1,0]$ case, denote $t_{2}^{*} \in[0,1]$ as the maximizer of $\max _{t \in[0,1]} t\left(R-D_{1+t}\left(P_{X Y} \| P_{X} \times Q_{Y}\right)\right)$. Then similarly we can have

$$
\begin{aligned}
& \liminf _{n \rightarrow \infty}-\frac{1}{n} \log D_{1+s}\left(P_{Y^{n} \mathcal{C}_{n}} \| Q_{Y}^{n} \times P_{\mathcal{C}_{n}}\right) \\
& \geq \liminf _{n \rightarrow \infty}-\frac{1}{n} \log D_{1+t_{2}^{*}}\left(P_{Y^{n} \mathcal{C}_{n}} \| Q_{Y}^{n} \times P_{\mathcal{C}_{n}}\right) \\
& \geq t_{2}^{*}\left(R-D_{1+t_{2}^{*}}\left(P_{X Y} \| P_{X} \times Q_{Y}\right)\right) \\
& =\max _{t \in[0,1]} t\left(R-D_{1+t}\left(P_{X Y} \| P_{X} \times Q_{Y}\right)\right) .
\end{aligned}
$$


Converse for $s \in(0,1]$ case: For the converse part, we follow steps similar to the proof in [12]. Let

$$
L\left(y^{n}\right):= \begin{cases}\frac{P_{Y}^{n}\left(y^{n}\right)}{Q_{Y}^{n}\left(y^{n}\right)} & \text { if } Q_{Y}^{n}\left(y^{n}\right)>0, \\ 1 & \text { otherwise, }\end{cases}
$$

denote the (random) likelihood ratio of each sequence $y^{n} \in$ $\mathcal{Y}^{n}$. Note that $P\left(y^{n}\right)$ is a random probability distribution, since the codebook is random. Since $P_{X} \in \mathcal{P}\left(P_{Y \mid X}, Q_{Y}\right)$, by the construction of the codebook, we have

$$
\mathbb{E}_{\mathcal{C}_{n}}\left[L\left(y^{n}\right)\right]=1, \quad \forall y^{n} \in \mathcal{Y}^{n} .
$$

Denote

$$
\ell(T):=\frac{P_{Y \mid X}^{n}\left(\tilde{y}^{n} \mid \tilde{x}^{n}\right)}{Q_{Y}^{n}\left(\tilde{y}^{n}\right)} \quad \text { for some }\left(\tilde{x}^{n}, \tilde{y}^{n}\right) \in \mathcal{T}_{T} .
$$

Denote

$$
N_{T}\left(y^{n}\right):=\left|\left\{x^{n} \in \mathcal{C}_{n}:\left(x^{n}, y^{n}\right) \in \mathcal{T}_{T}\right\}\right|
$$

as the number of codewords in $\mathcal{C}_{n}$ that have the joint type $T$ with $y^{n}$. Then $\left\{N_{T}\left(y^{n}\right): T \in \mathcal{P}^{(n)}(\mathcal{X} \times \mathcal{Y})\right\}$ is a collection of $M$ random variables with multinomial distributions and success probabilities

$$
p_{T}\left(y^{n}\right)=\mathbb{E}_{\mathcal{C}_{n}}\left[\frac{N_{T}\left(y^{n}\right)}{M}\right] .
$$

For brevity, here and in the following we denote $M=\mathrm{e}^{n R}$.

Partition $\mathcal{P}^{(n)}(\mathcal{X} \times \mathcal{Y})=\mathcal{P}_{1} \cup \mathcal{P}_{2}$ and split $L\left(y^{n}\right)=$ $L_{1}\left(y^{n}\right)+L_{2}\left(y^{n}\right)$, where

$$
\begin{aligned}
& \mathcal{P}_{1}:=\left\{T \in \mathcal{P}^{(n)}(\mathcal{X} \times \mathcal{Y}): \ell(T) \leq \mathrm{e}^{2} M\right\}, \\
& \mathcal{P}_{2}:=\left\{T \in \mathcal{P}^{(n)}(\mathcal{X} \times \mathcal{Y}): \ell(T)>\mathrm{e}^{2} M\right\},
\end{aligned}
$$

and

$$
\begin{aligned}
L_{1}\left(y^{n}\right) & :=\frac{1}{M} \sum_{T \in \mathcal{P}_{1}} N_{T}\left(y^{n}\right) \ell(T), \\
L_{2}\left(y^{n}\right) & :=\frac{1}{M} \sum_{T \in \mathcal{P}_{2}} N_{T}\left(y^{n}\right) \ell(T) .
\end{aligned}
$$

Hence

$$
\mathbb{E} L_{1}\left(y^{n}\right)+\mathbb{E} L_{2}\left(y^{n}\right)=1, \quad \forall y^{n} \in \mathcal{Y}^{n} .
$$

Also define

$$
\begin{aligned}
\nu\left(y^{n}\right) & :=\operatorname{Var}\left(L_{1}\left(y^{n}\right)\right)+\frac{1}{M} \mathbb{E}^{2}\left[L_{1}\left(y^{n}\right)\right], \text { and } \\
\mu\left(y^{n}\right) & :=\mathbb{E}\left[L_{2}\left(y^{n}\right)\right] .
\end{aligned}
$$

As in [12], by elementary properties of multinomial distribution one can show that

$$
\begin{aligned}
& \nu\left(y^{n}\right)=\frac{1}{M} \sum_{T \in \mathcal{P}_{1}} \ell(T)^{2} p_{T}\left(y^{n}\right), \\
& \mu\left(y^{n}\right)=\sum_{T \in \mathcal{P}_{2}} \ell(T) p_{T}\left(y^{n}\right) .
\end{aligned}
$$

Based on the above considerations, we have

$$
\begin{aligned}
& D_{1+s}\left(P_{Y^{n} \mathcal{C}_{n}} \| Q_{Y}^{n} \times P_{\mathcal{C}_{n}}\right) \\
& =\frac{1}{s} \log \left(\mathbb{E}_{\mathcal{C}_{n}} \sum_{y^{n}} Q\left(y^{n}\right) L^{1+s}\left(y^{n}\right)\right) \\
& \geq \frac{1}{s} \log \left(\mathbb{E}_{\mathcal{C}_{n}} \sum_{y^{n}} Q\left(y^{n}\right)\left(L_{1}^{1+s}\left(y^{n}\right)+L_{2}^{1+s}\left(y^{n}\right)\right)\right) \\
& =\frac{1}{s} \log \left(1+\mathbb{E}_{\mathcal{C}_{n}} \sum_{y^{n}} Q\left(y^{n}\right)\left(L_{1}^{1+s}\left(y^{n}\right)-L_{1}\left(y^{n}\right)\right.\right. \\
& \left.\left.\quad+L_{2}^{1+s}\left(y^{n}\right)-L_{2}\left(y^{n}\right)\right)\right) \\
& \quad \frac{1}{s} \mathbb{E}_{\mathcal{C}_{n}} \sum_{y^{n}} Q\left(y^{n}\right)\left(L_{1}^{1+s}\left(y^{n}\right)-L_{1}\left(y^{n}\right)\right. \\
& \left.\quad+L_{2}^{1+s}\left(y^{n}\right)-L_{2}\left(y^{n}\right)\right) \\
& \geq \mathbb{E}_{\mathcal{C}_{n}} \sum_{y^{n}} Q\left(y^{n}\right) L_{1}\left(y^{n}\right) \log L_{1}\left(y^{n}\right) \\
& \quad+\frac{1}{s} \mathbb{E}_{\mathcal{C}_{n}} \sum_{y^{n}} Q\left(y^{n}\right)\left(L_{2}^{1+s}\left(y^{n}\right)-L_{2}\left(y^{n}\right)\right) \\
& \quad+\sum_{y^{n}} Q\left(y^{n}\right)\left(\mathbb{E}_{\mathcal{C}_{n}} L_{1}\left(y^{n}\right) \log L_{1}\left(y^{n}\right)\right. \\
& \left.\quad+\mathbb{E}_{\mathcal{C}_{n}} \frac{1}{s}\left(L_{2}^{1+s}\left(y^{n}\right)-L_{2}\left(y^{n}\right)\right)\right) \\
& \geq \sum_{y^{n}} Q\left(y^{n}\right)\left(\frac{1}{M} \sum_{T \in \mathcal{P}_{1}} \ell^{2}(T) p_{T}\left(y^{n}\right)-\mathbb{E}_{\mathcal{C}_{n}} L_{2}\left(y^{n}\right)-\frac{1}{M}\left(L_{2}^{1+s}\left(y^{n}\right)-L_{2}\left(y^{n}\right)\right)\right)
\end{aligned}
$$

where (337) follows from Lemma 6, (339) follows from $\lim _{x \rightarrow 0} \frac{\log (1+x)}{x}=1$ and

$$
\begin{aligned}
& \mathbb{E}_{\mathcal{C}_{n}} \sum_{y^{n}} Q\left(y^{n}\right)\left(L_{1}^{1+s}\left(y^{n}\right)-L_{1}\left(y^{n}\right)\right. \\
&\left.+L_{2}^{1+s}\left(y^{n}\right)-L_{2}\left(y^{n}\right)\right) \rightarrow 0
\end{aligned}
$$

(this is obtained from the achievability part, where we have $D_{1+s}\left(P_{Y^{n} \mathcal{C}_{n}} \| Q_{Y}^{n} \times P_{\mathcal{C}_{n}}\right) \rightarrow 0$ ), (340) follows from $\frac{1}{s}\left(x^{1+s}-x\right) \geq x \log x$ (i.e., $\frac{1}{s}\left(x^{s}-1\right) \geq \log x$ ) for $s>0$ and $x \geq 0(0 \log 0:=0)$, and (342) follows from $\mathbb{E}_{\mathcal{C}_{n}} L_{1}\left(y^{n}\right) \log L_{1}\left(y^{n}\right) \geq \frac{1}{M} \sum_{T \in \mathcal{P}_{1}} \ell^{2}(T) p_{T}\left(y^{n}\right)-$ $\mathbb{E}_{\mathcal{C}_{n}} L_{2}\left(y^{n}\right)-\frac{1}{M}$ (which was proven in [12, Section V-C]).

Considering the last term in the bracket of (342), we have

$$
\begin{aligned}
& \frac{1}{s}\left(L_{2}^{1+s}\left(y^{n}\right)-L_{2}\left(y^{n}\right)\right) \\
& =\frac{\alpha+1-\alpha}{s}\left(L_{2}^{1+s}\left(y^{n}\right)-L_{2}\left(y^{n}\right)\right) \\
& \geq \alpha L_{2}\left(y^{n}\right) \log L_{2}\left(y^{n}\right)+\frac{1-\alpha}{s}\left(L_{2}^{1+s}\left(y^{n}\right)-L_{2}\left(y^{n}\right)\right) \\
& \geq 2 \alpha L_{2}\left(y^{n}\right)+\frac{1-\alpha}{s}\left(L_{2}^{1+s}\left(y^{n}\right)-L_{2}\left(y^{n}\right)\right) \\
& =\left(2 \alpha-1-\frac{1-\alpha}{s}\right) L_{2}\left(y^{n}\right)+\frac{1-\alpha}{s} L_{2}^{1+s}\left(y^{n}\right)
\end{aligned}
$$


where $\alpha \in[0,1]$ is an arbitrary number, (345) follows from $\frac{1}{s}\left(x^{1+s}-x\right) \geq x \log x$, and (346) follows from $L_{2}\left(y^{n}\right) \geq \mathrm{e}^{2}$.

Substitute (347) into (342), then we get

$$
\begin{aligned}
& D_{1+s}\left(P_{Y^{n} \mathcal{C}_{n}} \| Q_{Y}^{n} \times P_{\mathcal{C}_{n}}\right) \\
& \geq \sum_{y^{n}} Q\left(y^{n}\right)\left(\frac{1}{M} \sum_{T \in \mathcal{P}_{1}} \ell^{2}(T) p_{T}\left(y^{n}\right)\right. \\
& \quad+\left(2 \alpha-1-\frac{1-\alpha}{s}\right) \mathbb{E}_{\mathcal{C}_{n}} L_{2}\left(y^{n}\right) \\
& \left.\quad+\mathbb{E}_{\mathcal{C}_{n}} \frac{1-\alpha}{s} L_{2}^{1+s}\left(y^{n}\right)-\frac{1}{M}\right) .
\end{aligned}
$$

Choose $\alpha=\frac{1+s}{1+2 s}$, then the second term above vanishes. Hence we have

$$
\begin{aligned}
& D_{1+s}\left(P_{Y^{n} \mathcal{C}_{n}} \| Q_{Y}^{n} \times P_{\mathcal{C}_{n}}\right)+\frac{1}{M} \\
& =\sum_{y^{n}} Q\left(y^{n}\right)\left(\frac{1}{M} \sum_{T \in \mathcal{P}_{1}} \ell^{2}(T) p_{T}\left(y^{n}\right)\right. \\
& \left.+\mathbb{E}_{\mathcal{C}_{n}} \frac{1-\alpha}{s} L_{2}^{1+s}\left(y^{n}\right)\right) \\
& \doteq \sum_{y^{n}} Q\left(y^{n}\right)\left(\frac{1}{M} \sum_{T \in \mathcal{P}_{1}} \ell^{2}(T) p_{T}\left(y^{n}\right)\right. \\
& \left.+\mathbb{E}_{\mathcal{C}_{n}} L_{2}^{1+s}\left(y^{n}\right)\right) \\
& \geq \sum_{y^{n}} Q\left(y^{n}\right)\left(\frac{1}{M} \sum_{T \in \mathcal{P}_{1}} \ell^{2}(T) p_{T}\left(y^{n}\right)\right. \\
& \left.+\mathbb{E}_{\mathcal{C}_{n}} \sum_{T \in \mathcal{P}_{2}}\left(\frac{\ell(T)}{M}\right)^{1+s} N_{T}\left(y^{n}\right)\right) \\
& =\sum_{y^{n}} Q\left(y^{n}\right)\left(\frac{1}{M} \sum_{T \in \mathcal{P}_{1}} \ell^{2}(T) p_{T}\left(y^{n}\right)\right. \\
& \left.+\sum_{T \in \mathcal{P}_{2}}\left(\frac{\ell(T)}{M}\right)^{s} \ell(T) p_{T}\left(y^{n}\right)\right) \\
& \geq \sum_{y^{n}} Q\left(y^{n}\right)\left(\sum_{T \in \mathcal{P}^{(n)}(\mathcal{X} \times \mathcal{Y})} \ell(T) p_{T}\left(y^{n}\right)\right. \\
& \left.\times \min \left\{\frac{\ell(T)}{M},\left(\frac{\ell(T)}{M}\right)^{s}\right\}\right) \text {. }
\end{aligned}
$$

where (351) follows from that

$$
\begin{aligned}
L_{2}^{1+s}\left(y^{n}\right) & =\left(\sum_{T \in \mathcal{P}_{2}} \frac{\ell(T) N_{T}\left(y^{n}\right)}{M}\right)^{1+s} \\
& =\sum_{T \in \mathcal{P}_{2}} \frac{\ell(T) N_{T}\left(y^{n}\right)}{M}\left(\sum_{T^{\prime} \in \mathcal{P}_{2}} \frac{\ell\left(T^{\prime}\right) N_{T^{\prime}}\left(y^{n}\right)}{M}\right)^{s} \\
& \geq \sum_{T \in \mathcal{P}_{2}}\left(\frac{\ell(T) N_{T}\left(y^{n}\right)}{M}\right)^{1+s} \\
& \geq \sum_{T \in \mathcal{P}_{2}}\left(\frac{\ell(T)}{M}\right)^{1+s} N_{T}\left(y^{n}\right) .
\end{aligned}
$$

Following steps similar to (111)-(121) of [12], we can get

$$
\begin{aligned}
& D_{1+s}\left(P_{Y^{n} \mathcal{C}_{n}} \| Q_{Y}^{n} \times P_{\mathcal{C}_{n}}\right) \\
& \dot{\sum_{T \in \mathcal{P}^{(n)}(\mathcal{X} \times \mathcal{Y})}} \mathrm{e}^{-n D\left(T \| T_{X} \times P_{Y \mid X}\right)} P_{X^{n}}\left(\mathcal{T}_{T_{X}}\right) \\
& \quad \times \min \left\{\frac{\ell(T)}{M},\left(\frac{\ell(T)}{M}\right)^{s}\right\} .
\end{aligned}
$$

Note that (358) holds for all random codes such that

$$
\mathbb{E}\left[P\left(y^{n}\right)\right]=Q\left(y^{n}\right), \quad \forall y^{n} \in \mathcal{Y}^{n} .
$$

Moreover, for the ensemble of i.i.d. random codes, we have

$$
P_{X^{n}}\left(\mathcal{T}_{T_{X}}\right) \doteq \mathrm{e}^{-n D\left(T_{X} \| P_{X}\right)}
$$

and

$$
\begin{aligned}
& \min \left\{\frac{\ell(T)}{M},\left(\frac{\ell(T)}{M}\right)^{s}\right\} \\
& \doteq \mathrm{e}^{-n \max \left\{R-f\left(T \| P_{X Y}\right), s\left(R-f\left(T \| P_{X Y}\right)\right)\right\}}
\end{aligned}
$$

where

$$
f\left(P \| P^{\prime}\right):=\sum_{(x, y) \in \mathcal{X} \times \mathcal{Y}} P(x, y) \log \frac{P^{\prime}(x, y)}{P_{X}^{\prime}(y) P_{Y}^{\prime}(y)},
$$

for any two distributions $P, P^{\prime} \in \mathcal{P}(\mathcal{X} \times \mathcal{Y})$. Therefore,

$$
\begin{aligned}
& D_{1+s}\left(P_{Y^{n} \mathcal{C}_{n}} \| Q_{Y}^{n} \times P_{\mathcal{C}_{n}}\right) \\
& \dot{\exp }\left\{-n \min _{T}\left\{D\left(T \| T_{X} \times P_{Y \mid X}\right)+D\left(T_{X} \| P_{X}\right)\right.\right. \\
& \left.\left.\quad+\max \left\{R-f\left(T \| P_{X Y}\right), s\left(R-f\left(T \| P_{X Y}\right)\right)\right\}\right\}\right\} \\
& =\mathrm{e}^{-n \min _{T}\left\{D\left(T \| P_{X Y}\right)+\max \left\{R-f\left(T \| P_{X Y}\right), s\left(R-f\left(T \| P_{X Y}\right)\right)\right\}\right\}} .
\end{aligned}
$$

Furthermore, we can get

$$
\begin{aligned}
& \quad \min _{T \in \mathcal{P}^{(n)}(\mathcal{X} \times \mathcal{Y})}\left\{D\left(T \| P_{X Y}\right)\right. \\
& \left.\quad+\max \left\{R-f\left(T \| P_{X Y}\right), s\left(R-f\left(T \| P_{X Y}\right)\right)\right\}\right\} \\
& =\min _{\widetilde{P} \in \mathcal{P}(\mathcal{X} \times \mathcal{Y})}\left\{D\left(\widetilde{P} \| P_{X Y}\right)\right. \\
& \left.\quad+\max \left\{R-f\left(\widetilde{P} \| P_{X Y}\right), s\left(R-f\left(\widetilde{P} \| P_{X Y}\right)\right)\right\}\right\}+\delta_{n} \\
& =\max _{t \in[s, 1]} t\left(R-D_{1+t}\left(Q_{X Y} \| Q_{X} Q_{Y}\right)\right)+\delta_{n},
\end{aligned}
$$

where (365) follows from Lemma 4, and (366) is obtained by following steps similar to the proof in Appendix B-D of [12]. Hence we have for i.i.d. codes,

$$
\begin{aligned}
& \limsup _{n \rightarrow \infty}-\frac{1}{n} \log D_{1+s}\left(P_{Y^{n} \mathcal{C}_{n}} \| Q_{Y}^{n} P_{\mathcal{C}_{n}}\right) \\
& \leq \max _{t \in[s, 1]} t\left(R-D_{1+t}\left(Q_{X Y} \| Q_{X} Q_{Y}\right)\right) .
\end{aligned}
$$

Converse for $s \in(-1,0]$ case: For this case, we need to prove for $s \in[0,1)$,

$$
\begin{aligned}
& \limsup _{n \rightarrow \infty}-\frac{1}{n} \log D_{1-s}\left(P_{Y^{n} \mathcal{C}_{n}} \| Q_{Y}^{n} \times P_{\mathcal{C}_{n}}\right) \\
& \leq \max _{t \in[0,1]} t\left(R-D_{1+t}\left(Q_{X Y} \| Q_{X} \times Q_{Y}\right)\right) .
\end{aligned}
$$


We also follow steps similar to the proof in [12], and still use the notations (322)-(335), but we need to instead choose

$$
\begin{aligned}
& \mathcal{P}_{1}:=\left\{T \in \mathcal{P}^{(n)}(\mathcal{X} \times \mathcal{Y}): \ell(T) \leq \beta M\right\} \\
& \mathcal{P}_{2}:=\left\{T \in \mathcal{P}^{(n)}(\mathcal{X} \times \mathcal{Y}): \ell(T)>\beta M\right\}
\end{aligned}
$$

for some $\beta>0$. Then we have

$$
\begin{aligned}
& D_{1-s}\left(P_{Y^{n} \mathcal{C}_{n}} \| Q_{Y}^{n} \times P_{\mathcal{C}_{n}}\right) \\
& \dot{\geq}-\frac{1}{s} \mathbb{E}_{\mathcal{C}_{n}} \sum_{y^{n}} Q\left(y^{n}\right)\left(L_{1}^{1-s}\left(y^{n}\right)-L_{1}\left(y^{n}\right)\right. \\
& \left.+L_{2}^{1-s}\left(y^{n}\right)-L_{2}\left(y^{n}\right)\right) \\
& \geq \sum_{y^{n}} Q\left(y^{n}\right)\left(-\mathbb{E}_{\mathcal{C}_{n}}\left(\frac{L_{1}^{1-s}\left(y^{n}\right)-L_{1}\left(y^{n}\right)}{s}\right)\right. \\
& \left.-\frac{1}{s} \mathbb{E}_{\mathcal{C}_{n}}\left(\beta^{-s} L_{2}\left(y^{n}\right)-L_{2}\left(y^{n}\right)\right)\right) \\
& =\sum_{y^{n}} Q\left(y^{n}\right)\left(\mathbb{E}_{\mathcal{C}_{n}} \frac{L_{1}-L_{1}^{1-s}}{s}+\frac{1-\beta^{-s}}{s} \mathbb{E}_{\mathcal{C}_{n}} L_{2}\right) \text {. }
\end{aligned}
$$

where (371) is obtained by following steps similar to (336)(339), and (372) follows from $L_{2}\left(y^{n}\right) \geq \beta$.

To continue the proof, we need the following lemma. The proof is similar as that of [12, Lemma 7], and hence omitted here.

Lemma 9. Let $U$ be an arbitrary non-negative random variable with $\mathbb{E}[U]=1$. Then, for any $\theta>0$,

$$
c(\theta)\left[\operatorname{Var}(U)-\tau_{\theta}(U)\right] \leq \mathbb{E}\left[\frac{U-U^{1-s}}{s}\right] \leq \operatorname{Var}(U)
$$

where

$$
\tau_{\theta}(U):=\theta^{2} \mathbb{P}\{U>(\theta+1)\}+2 \int_{\theta}^{+\infty} v \mathbb{P}\{U>v+1\} \mathrm{d} v,
$$

and

$$
c(\theta):=\frac{1}{\theta^{2}}\left(\frac{\theta+1-(\theta+1)^{1-s}}{s}-\theta\right) .
$$

Using this lemma, we have for all $\theta>0$,

$$
\begin{aligned}
& \mathbb{E}\left[\frac{\left.L_{1}-L_{1}^{1-s}\right]}{s}\right] \\
& =\mathbb{E}\left[\frac{L_{1}-\mathbb{E}\left[L_{1}\right]^{s} L_{1}^{1-s}+\mathbb{E}\left[L_{1}\right]^{s} L_{1}^{1-s}-L_{1}^{1-s}}{s}\right] \\
& =\mathbb{E}\left[L_{1}\right] \mathbb{E}\left[\frac{1}{s}\left(\frac{L_{1}}{\mathbb{E} L_{1}}-\left(\frac{L_{1}}{\mathbb{E} L_{1}}\right)^{1-s}\right)\right] \\
& \quad+\frac{\left(\mathbb{E}\left[L_{1}\right]^{s}-1\right) \mathbb{E}\left[L_{1}^{1-s}\right]}{s} \\
& \geq \mathbb{E}\left[L_{1}\right] c(\theta)\left[\operatorname{Var}\left(U_{1}\right)-\tau_{\theta}\left(U_{1}\right)\right]-\mathbb{E}\left[L_{2}\right],
\end{aligned}
$$

where $U_{1}:=\frac{L_{1}}{\mathbb{E} L_{1}}$ and (379) follows from the lemma above and the following inequalities.

$$
\begin{aligned}
& \frac{\left(\mathbb{E}\left[L_{1}\right]^{s}-1\right) \mathbb{E}\left[L_{1}^{1-s}\right]}{s} \\
& \geq \frac{\left(\mathbb{E}\left[L_{1}\right]^{s}-1\right) \mathbb{E}\left[L_{1}\right]^{1-s}}{s} \\
& =\frac{\left(\mathbb{E}\left[L_{1}\right]-\mathbb{E}\left[L_{1}\right]^{1-s}\right)}{s} \\
& \geq \mathbb{E}\left[L_{1}\right]-1 \\
& =-\mathbb{E}\left[L_{2}\right],
\end{aligned}
$$

where (380) follows from the fact that $x \mapsto x^{1-s}$ is a concave function, and $\mathbb{E} L_{1} \leq 1$, and (382) follows since $\frac{1}{s}\left(x-x^{1-s}\right) \geq x-1$ for $s \in[0,1)$ and $x \in[0,1]$.

Using (373) and (379) we obtain that $\forall \theta>0$ :

$$
\begin{aligned}
& D_{1-s}\left(P_{Y^{n} \mathcal{C}_{n}} \| Q_{Y}^{n} \times P_{\mathcal{C}_{n}}\right) \\
& \geq \mathbb{E}\left[L_{1}\right] c(\theta)\left[\operatorname{Var}\left(U_{1}\right)-\tau_{\theta}\left(U_{1}\right)\right]+\frac{1-\beta^{-s}-s}{s} \mathbb{E}\left[L_{2}\right] .
\end{aligned}
$$

Furthermore, choose $\beta>\left(\frac{1}{1-s}\right)^{s}$, then $\frac{1-\beta^{-s}-s}{s}>0$. Hence

$$
\begin{aligned}
& D_{1-s}\left(P_{Y^{n} \mathcal{C}_{n}} \| Q_{Y}^{n} \times P_{\mathcal{C}_{n}}\right) \\
& \geq \mathbb{E}\left[L_{1}\right] c(\theta)\left[\operatorname{Var}\left(U_{1}\right)-\tau_{\theta}\left(U_{1}\right)\right]+\mathbb{E}\left[L_{2}\right] .
\end{aligned}
$$

Then we can get

$$
\begin{aligned}
& D_{1-s}\left(P_{Y^{n} \mathcal{C}_{n}} \| Q_{Y}^{n} \times P_{\mathcal{C}_{n}}\right)+\frac{1}{M} \\
& \geq \nu+\mu \\
& \doteq \mathrm{e}^{-n} \min _{T \in \mathcal{P}^{(n)}(\mathcal{X} \times \mathcal{Y})}\left(D\left(T \| T_{X} P_{Y \mid X}\right)+D\left(T_{X} \| P_{X}\right)+\left[R-f\left(T \| P_{X Y}\right)\right]^{+}\right)
\end{aligned}
$$

$=\mathrm{e}^{-n} \min _{T \in \mathcal{P}^{(n)}(\mathcal{X} \times \mathcal{Y})}\left(D\left(T \| P_{X Y}\right)+\left[R-f\left(T \| P_{X Y}\right)\right]^{+}\right)$

$\doteq \mathrm{e}^{-n \min _{\widetilde{P} \in \mathcal{P}(\mathcal{X} \times \mathcal{Y})}\left(D\left(\widetilde{P} \| P_{X Y}\right)+\left[R-f\left(\widetilde{P} \| P_{X Y}\right)\right]^{+}\right)}$

$=\mathrm{e}^{-n \max _{t \in[0,1]} t\left(R-D_{1+t}\left(Q_{X Y} \| Q_{X} \times Q_{Y}\right)\right)}$

where (385) is obtained by following steps similar to (101)(125) of [12], $f(\cdot)$ is defined in (362), (386) follows from [12, Eqns. (122)-(125)], (388) follows from Lemma 4 (or [12, Appendix B-A]), and (389) follows from [12, Appendix B-D].

Since the exponent of $\frac{1}{M}$ is $R$, which is larger than the exponent in (389), the exponent in (389) is the dominant exponent. Hence (389) implies the converse part.

\section{APPENDIX G}

PROOF OF THEOREM 4

The achievability of $\mathrm{E}_{\mathrm{iid}}\left(P_{X}, P_{Y \mid X}, Q_{Y}\right)$ has been proven in Theorem 3, hence we only need to prove the achievability of $\mathrm{E}_{\mathrm{ts}}\left(P_{X}, P_{Y \mid X}, Q_{Y}\right)$.

For the case of $s \in(-1,0]$, the exponent $\sup _{\epsilon \in(0,1]} \min \left\{\frac{\epsilon^{2} P_{\min }}{3}, \theta\left(0, \epsilon, P_{X}\right)\right\} \quad$ is obtained from the exponent for $s \in(0,1]$ by letting $s \rightarrow 0$. Hence we only need to focus on the case $s \in(0,1]$. We use the 
random code given in the proof of Theorem 2. For this code, $P_{X^{n}}\left(x^{n}\right) \propto Q_{X}^{n}\left(x^{n}\right) 1\left\{x^{n} \in \mathcal{T}_{\epsilon}^{n}\left(Q_{X}\right)\right\}$ for some $Q_{X} \in \mathcal{P}\left(P_{Y \mid X}, Q_{Y}\right)$.

By Lemma 1, we obtain

$$
\begin{aligned}
& \mathrm{e}^{s D_{1+s}\left(P_{Y^{n}} \mathcal{C}_{n} \| Q_{Y}^{n} \times P_{\mathcal{C}_{n}}\right)} \\
& \leq \mathrm{e}^{s D_{1+s}\left(P_{X^{n}} Y^{n} \| P_{X^{n}} Q_{Y}^{n}\right)-n s R}+\mathrm{e}^{s D_{1+s}\left(P_{Y} n \| Q_{Y}^{n}\right)} \\
& =\mathrm{e}^{s D_{1+s}\left(P_{Y^{n}} \| Q_{Y}^{n}\right)} \\
& \times\left(\mathrm{e}^{s D_{1+s}\left(P_{X^{n} Y^{n}} \| P_{X^{n}} Q_{Y}^{n}\right)-n s R-s D_{1+s}\left(P_{Y} n \| Q_{Y}^{n}\right)}+1\right) .
\end{aligned}
$$

Take log's,

$$
\begin{aligned}
& D_{1+s}\left(P_{Y^{n}} \mathcal{C}_{n} \| Q_{Y}^{n} \times P_{\mathcal{C}_{n}}\right) \\
& =D_{1+s}\left(P_{Y^{n}} \| Q_{Y}^{n}\right) \\
& +\frac{1}{s} \log \left(\mathrm{e}^{s D_{1+s}\left(P_{X^{n} Y^{n}} \| P_{X^{n}} Q_{Y}^{n}\right)-n s R-s D_{1+s}\left(P_{Y^{n}} \| Q_{Y}^{n}\right)}+1\right) \\
& \leq D_{1+s}\left(P_{Y^{n}} \| Q_{Y}^{n}\right) \\
& +\frac{1}{s} \mathrm{e}^{s D_{1+s}\left(P_{X^{n} Y^{n}} \| P_{X^{n}} Q_{Y}^{n}\right)-n s R-s D_{1+s}\left(P_{Y^{n}} \| Q_{Y}^{n}\right)}
\end{aligned}
$$

On the other hand,

$$
\begin{aligned}
D_{1+s}\left(P_{Y^{n}} \| Q_{Y}^{n}\right) & \leq D_{1+s}\left(P_{X^{n}} \| Q_{X}^{n}\right) \\
& =\log \frac{1}{Q_{X}^{n}\left(\mathcal{T}_{\epsilon}^{n}\right)} \\
& \leq \frac{1}{Q_{X}^{n}\left(\mathcal{T}_{\epsilon}^{n}\right)}-1 \\
& \doteq Q_{X}^{n}\left(\left(\mathcal{T}_{\epsilon}^{n}\right)^{c}\right),
\end{aligned}
$$

where $\left(\mathcal{T}_{\epsilon}^{n}\right)^{c}:=\mathcal{X}^{n} \backslash \mathcal{T}_{\epsilon}^{n}$, and (395) follows from (274)-(276). Now we bound $Q_{X}^{n}\left(\left(\mathcal{T}_{\epsilon}^{n}\right)^{c}\right)$ using the Chernoff bound [54] as

$$
Q_{X}^{n}\left(\left(\mathcal{T}_{\epsilon}^{n}\right)^{c}\right) \leq 2|\mathcal{X}| \mathrm{e}^{-\frac{\epsilon^{2} n Q_{\min }}{3}},
$$

where $Q_{\min }:=\min _{x} Q_{X}(x)$. Substituting (398) into (397), we obtain

$$
D_{1+s}\left(P_{Y^{n}} \| Q_{Y}^{n}\right) \dot{\leq} 2|\mathcal{X}| \mathrm{e}^{-\frac{\epsilon^{2} n Q_{\min }}{3}} .
$$

By (399) we can bound the exponent of the second term of (393) as

$$
\begin{aligned}
& s R-\frac{1}{n} s D_{1+s}\left(P_{X^{n} Y^{n}} \| P_{X^{n}} Q_{Y}^{n}\right)+\frac{1}{n} s D_{1+s}\left(P_{Y^{n}} \| Q_{Y}^{n}\right) \\
& =s R+\delta_{n}-\frac{1}{n} \log \sum_{x^{n} \in \mathcal{T}_{\epsilon}^{n}} P\left(x^{n}\right) \\
& \quad \times \mathrm{e}^{n \sum_{x \in \mathcal{X}} T_{x}^{n}(x) \log \sum_{y} P^{1+s}(y \mid x) Q^{-s}(y)} \\
& =s R+\delta_{n}-\frac{1}{n} \log \sum_{T_{X}: \forall x:\left|T_{X}(x)-Q(x)\right| \leq \epsilon Q(x)} P_{X^{n}}\left(\mathcal{T}_{T_{X}}\right) \\
& \quad \times \mathrm{e}^{n \sum_{x \in \mathcal{X}} T_{X}(x) \log \sum_{y} P^{1+s}(y \mid x) Q^{-s}(y)} \\
& \geq s R+\delta_{n}-T_{X}: \forall x:\left|T_{X}(x)-Q(x)\right| \leq \epsilon Q(x) \\
& \quad \times \log \sum_{y} P^{1+s}(y \mid x) T_{X}^{-s}(y) \\
& \geq s R+\delta_{n}-(1+\epsilon) \sum_{x} Q(x) \log \sum_{y} P^{1+s}(y \mid x) Q^{-s}(y),
\end{aligned}
$$

where $\delta_{n}$ is a term vanishing as $n \rightarrow \infty$, and (402) follows since $\sum_{T_{X}: \forall x:\left|T_{X}(x)-Q(x)\right| \leq \epsilon Q(x)} P_{X^{n}}\left(\mathcal{T}_{T_{X}}\right) \leq 1$ and for any $T_{X}$ such that for all $x,\left|T_{X}(x)-Q(x)\right| \leq \epsilon Q(x)$, it holds that

$$
\begin{aligned}
& \sum_{x \in \mathcal{X}} T_{X}(x) \log \left(\sum_{y} P^{1+s}(y \mid x) Q^{-s}(y)\right) \\
& \leq \max _{T_{X}: \forall x:\left|T_{X}(x)-Q(x)\right| \leq \epsilon Q(x)} \sum_{x \in \mathcal{X}} T_{X}(x) \\
& \quad \times \log \sum_{y} P^{1+s}(y \mid x) Q^{-s}(y) .
\end{aligned}
$$

Substituting (399) and (403) into (393), we have

$$
\begin{aligned}
& \liminf _{n \rightarrow \infty}-\frac{1}{n} \log D_{1+s}\left(P_{Y^{n} \mathcal{C}_{n}} \| Q_{Y}^{n} \times P_{\mathcal{C}_{n}}\right) \\
& \geq \min \left\{\frac{\epsilon^{2} Q_{\min }}{3},\right. \\
& \left.\quad s R-(1+\epsilon) \sum_{x} Q(x) \log \sum_{y} P^{1+s}(y \mid x) Q^{-s}(y)\right\} .
\end{aligned}
$$

Note that the second term of minimization is not $\theta\left(s, \epsilon, P_{X}\right)$. To obtain the desired result, by using the fact that the Rényi divergence is non-decreasing in its parameter, we get

$$
\begin{aligned}
\liminf _{n \rightarrow \infty}-\frac{1}{n} \log D_{1+s}\left(P_{Y^{n}} \mathcal{C}_{n} \| Q_{Y}^{n} \times P_{\mathcal{C}_{n}}\right) \\
\geq \sup _{t \in[s, 1]} \liminf _{n \rightarrow \infty}-\frac{1}{n} \log D_{1+t}\left(P_{Y^{n} \mathcal{C}_{n}} \| Q_{Y}^{n} \times P_{\mathcal{C}_{n}}\right) \\
\geq \sup _{t \in[s, 1]} \min \left\{\frac{\epsilon^{2} Q_{\min }}{3},\right. \\
\left.\quad t R-(1+\epsilon) \sum_{x} Q(x) \log \sum_{y} P^{1+t}(y \mid x) Q^{-t}(y)\right\} \\
=\min \left\{\frac{\epsilon^{2} Q_{\min }}{3}, \theta\left(s, \epsilon, P_{X}\right)\right\} .
\end{aligned}
$$

Since $\epsilon \in(0,1]$ is arbitrary, we can optimize (408) over all possible $\epsilon$. This concludes the proof.

\section{APPENDIX H \\ ProOF OF THEOREM 5}

Achievability: We use random coding to prove the achievability part. Generate $\mathcal{C}_{n}=\left\{X^{n}\left(m_{0}, m_{1}\right)\right\}_{\left(m_{0}, m_{1}\right) \in \mathcal{M}_{0} \times \mathcal{M}_{1}}$ with $X^{n}\left(m_{0}, m_{1}\right) \sim P_{X^{n}}$ and set the encoder as $f_{\mathcal{C}_{n}}\left(m_{0}, m_{1}\right)=X^{n}\left(m_{0}, m_{1}\right)$. This constitutes our random code. Moreover, we set $P_{X^{n}}\left(x^{n}\right) \propto$ $P_{X}^{n}\left(x^{n}\right) 1\left\{x^{n} \in \mathcal{T}_{\epsilon}^{n}\left(P_{X}\right)\right\}$ for some $P_{X} \in \mathcal{P}\left(P_{Z \mid X}, Q_{Z}\right)$. At the legitimate user side, the standard joint-typicality decoder is adopted. 
For this random code, by the standard proof [55, Section $3.1 .2]^{10}$, it is easy to verify that

$$
\begin{aligned}
& \mathbb{P}\left(\left(M_{0}, M_{1}\right) \neq\left(\widehat{M}_{0}, \widehat{M}_{1}\right)\right) \\
& =\mathbb{E}_{\mathcal{C}_{n}}\left[\mathbb{P}\left(\left(M_{0}, M_{1}\right) \neq\left(\widehat{M}_{0}, \widehat{M}_{1}\right) \mid \mathcal{C}_{n}\right)\right] \\
& \rightarrow 0
\end{aligned}
$$

if $R_{0}+R_{1} \leq I(X ; Y)$. Therefore, the error constraint is satisfied.

By the codebook generation procedure, $\mathcal{C}_{n}$ is independent of $M_{1}$, and the subcodebooks $\mathcal{C}_{n}\left(m_{1}\right)$ := $\left\{X^{n}\left(m_{0}, m_{1}\right)\right\}_{m_{0} \in \mathcal{M}_{0}}$ for different $m_{1}$ have the same distribution (which implies $P_{\mathcal{C}_{n}\left(m_{1}\right)}\left(c_{n}\right)=P_{\mathcal{C}_{n}\left(M_{1}\right)}\left(c_{n}\right)$ for any $\left.m_{1}\right)$. Hence $\mathcal{C}_{n}\left(M_{1}\right)$ is independent of $M_{1} \cdot{ }^{11}$ Furthermore, from our result for the channel resolvability problem (Theorem 2), given $M_{1}=m_{1}$ and $P_{X} \in \mathcal{P}\left(P_{Z \mid X}, Q_{Z}\right)$, for $s \in[-1,1]$, the random code constructed above satisfies

$$
D_{1+s}\left(P_{Z^{n} \mathcal{C}_{n}\left(m_{1}\right) \mid M_{1}=m_{1}} \| Q_{Z}^{n} \times P_{\mathcal{C}_{n}\left(m_{1}\right)}\right) \rightarrow 0
$$

if $R_{0}>\widetilde{R}_{1+s}\left(P_{X}, P_{Z \mid X}, Q_{Z}\right)$. Therefore,

$$
\begin{aligned}
& \mathrm{e}^{s D_{1+s}\left(P_{Z^{n} M_{1} \mathcal{c}_{n}} \| Q_{Z}^{n} \times P_{M_{1} \mathcal{C}_{n}}\right)} \\
& =\mathbb{E}_{M_{1} \mathcal{C}_{n}}\left[\mathrm{e}^{s D_{1+s}\left(P_{Z^{n} \mid M_{1} \mathcal{c}_{n}} \| Q_{Z}^{n}\right)}\right] \\
& =\mathbb{E}_{M_{1}, \mathcal{C}_{n}\left(M_{1}\right)}\left[\mathrm{e}^{s D_{1+s}\left(P_{Z^{n} \mid M_{1}, \mathcal{C}_{n}\left(M_{1}\right)} \| Q_{Z}^{n}\right)}\right] \\
& =\mathbb{E}_{M_{1}}\left[\mathrm{e}^{s D_{1+s}\left(P_{Z^{n}} \mathcal{C}_{n}\left(M_{1}\right) \mid M_{1} \| Q_{Z}^{n} \times P_{\mathcal{C}_{n}\left(M_{1}\right)}\right)}\right] \\
& \rightarrow 1 \text {, }
\end{aligned}
$$

where (413) follows since $\mathcal{C}_{n} \rightarrow\left(M_{1}, \mathcal{C}_{n}\left(M_{1}\right)\right) \rightarrow Z^{n}$ forms a Markov chain (this results from the encoding process-the transmitted codeword is chosen from $\mathcal{C}_{n}\left(M_{1}\right)$ ), and (414) follows since $M_{1}$ and $\mathcal{C}_{n}\left(M_{1}\right)$ are independent. On the other hand,

$$
\begin{aligned}
& \mathrm{e}^{s D_{1+s}\left(P_{Z^{n}, M_{1}, \mathcal{c}_{n}} \| Q_{Z}^{n} \times P_{M_{1}, \mathcal{C}_{n}}\right)} \\
& =\mathbb{E}_{\mathcal{C}_{n}}\left[\mathrm{e}^{s D_{1+s}\left(P_{Z^{n} M_{1}} \| Q_{Z}^{n} \times P_{M_{1}}\right)}\right] .
\end{aligned}
$$

Hence

$$
\mathbb{E}_{\mathcal{C}_{n}}\left[\mathrm{e}^{s D_{1+s}\left(P_{Z^{n} M_{1}} \| Q_{Z}^{n} \times P_{M_{1}}\right)}\right] \rightarrow 1 .
$$

Applying the selection lemma [56, Lem. 2.2] to (410) and (417) we deduce that there exists one sequence of realizations $\left\{c_{n}\right\}_{n}$ such that given $\mathcal{C}_{n}=c_{n}$,

$$
\lim _{n \rightarrow \infty} \mathbb{P}\left(\left(M_{0}, M_{1}\right) \neq\left(\widehat{M}_{0}, \widehat{M}_{1}\right)\right)=0,
$$

and

$$
\lim _{n \rightarrow \infty} D_{1+s}\left(P_{M_{1} Z^{n}} \| P_{M_{1}} Q_{Z}^{n}\right)=0 .
$$

Hence $f_{\mathcal{C}_{n}=c_{n}}$ is the desired encoder. The proof of the achievability part for $s \in[-1,1]$ is complete. For $s=\infty$, the achievability part can be proven similarly.

\footnotetext{
${ }^{10}$ Although here $P_{X^{n}}$ is not an i.i.d. distribution, it satisfies $P_{X^{n}}\left(x^{n}\right)=$ $\mathrm{e}^{-n\left(H\left(P_{X}\right)+\delta_{n}\right)}$. Hence the joint typicality lemma [55] still holds, which further guarantees that the standard proof for channel coding works for our case.

${ }^{11}$ Indeed, we have $\quad P_{M_{1}, \mathcal{C}_{n}\left(M_{1}\right)}\left(m_{1}, c_{n}\right)$ =

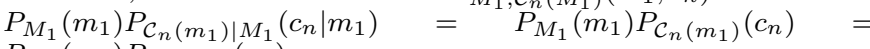
$P_{M_{1}}\left(m_{1}\right) P_{\mathcal{C}_{n}\left(M_{1}\right)}\left(c_{n}\right)$.
}

Converse: By the data processing inequality [35], we have

$$
\begin{aligned}
R_{0}+R_{1} & \leq \frac{1}{n} I\left(X^{n} ; Y^{n}\right) \\
& \leq I\left(X_{J} ; Y_{J}\right),
\end{aligned}
$$

where $J \sim$ Unif $[1: n]$ denotes a time index variable, independent of $X^{n}, Y^{n}$. It is easy to verify that the distribution of $\left(X_{J}, Y_{J}\right)$ induced by an $n$-length code satisfies

$$
\begin{aligned}
P_{X_{J} Y_{J}}^{(n)}(x, y) & =\mathbb{E}_{X^{n} Y^{n}}\left[T_{X^{n} Y^{n}}(x, y)\right] \\
& =\mathbb{E}_{X^{n}}\left[T_{X^{n}}(x)\right] P(y \mid x) .
\end{aligned}
$$

Now, Pinsker's inequality for Rényi parameter $1+s \in$ $(0,1]$ [35] implies that,

$$
|P-Q| \leq \sqrt{\frac{2}{1+s} D_{1+s}(P \| Q)},
$$

and for Rényi parameter $1+s \in(1, \infty]$, we also have

$$
|P-Q| \leq \sqrt{2 D(P \| Q)} \leq \sqrt{2 D_{1+s}(P \| Q)} .
$$

Applying (424) and (425) to $P_{M_{1} Z^{n}}$ and $P_{M_{1}} Q_{Z}^{n}$, we obtain $\left|P_{M_{1} Z^{n}}-P_{M_{1}} Q_{Z}^{n}\right| \rightarrow 0$ and hence $\left|P_{Z^{n}}-Q_{Z}^{n}\right| \rightarrow 0$ (by the data processing inequality of TV distance $\left|P_{X}-Q_{X}\right| \leq$ $\left.\left|P_{X Y}-Q_{X Y}\right|\right)$, regardless of $1+s \in(0,1]$ or $(1,2]$.

Observe that $\left|T_{Z^{n}}-Q_{Z}\right|$ is a function of $Z^{n}$ and upperbounded by 1 , and $\mathbb{E}_{Q_{Z}^{n}}\left|T_{Z^{n}}-Q_{Z}\right| \rightarrow 0$ as $n \rightarrow \infty$. Hence by the property

$$
\sup _{f: \mathcal{X} \rightarrow[0,1]}\left|\mathbb{E}_{P} f(X)-\mathbb{E}_{Q} f(X)\right|=|P-Q|,
$$

we have

$$
\lim _{n \rightarrow \infty} \mathbb{E}_{P_{Z^{n}}}\left|T_{Z^{n}}-Q_{Z}\right|=\lim _{n \rightarrow \infty} \mathbb{E}_{Q_{Z}^{n}}\left|T_{Z^{n}}-Q_{Z}\right|=0,
$$

which further implies

$$
\lim _{n \rightarrow \infty}\left|\mathbb{E}_{P_{Z^{n}}}\left[T_{Z^{n}}\right]-Q_{Z}\right|=0
$$

i.e.,

$$
\lim _{n \rightarrow \infty}\left|P_{Z_{J}}^{(n)}-Q_{Z}\right|=0
$$

or equivalently

$$
\lim _{n \rightarrow \infty}\left|P_{X_{J}}^{(n)} \circ P_{Z \mid X}-Q_{Z}\right|=0 .
$$

Since $\mathcal{P}(\mathcal{X})$ is compact, there must exist some increasing sequence $\left\{n_{k}\right\}_{k=1}^{\infty}$ such that $P_{X_{J}}^{\left(n_{k}\right)}$ converges to some distribution $\widetilde{P}_{X}$. From (430), $\widetilde{P}_{X} \in \mathcal{P}\left(P_{Z \mid X}, Q_{Z}\right)$ holds.

We first consider the case of $s \in(0, \infty]$. By the one-shot bound in Lemma 2,

$$
\lim _{n \rightarrow \infty} D_{1+s}\left(P_{M_{1} Z^{n}} \| P_{M_{1}} Q_{Z}^{n}\right)=0
$$

implies

$$
R_{0} \geq \limsup _{n \rightarrow \infty} \frac{1}{n} D_{1+s}\left(P_{X^{n} Z^{n}} \| P_{X^{n}} \times Q_{Z}^{n}\right) .
$$


On the other hand,

$$
\begin{aligned}
& \frac{1}{n} D_{1+s}\left(P_{X^{n}} Z^{n} \| P_{X^{n}} \times Q_{Z}^{n}\right) \\
& =\frac{1}{n s} \log \sum_{T_{X}} P_{X^{n}}\left(\mathcal{T}_{T_{X}}\right) \mathrm{e}^{n \sum_{x \in \mathcal{X}} T_{X}(x) \log \sum_{z} P^{1+s}(z \mid x) Q^{-s}(z)} \\
& \geq \frac{1}{s} \sum_{T_{X}} P_{X^{n}}\left(\mathcal{T}_{T_{X}}\right) \sum_{x \in \mathcal{X}} T_{X}(x) \log \sum_{z} P^{1+s}(z \mid x) Q^{-s}(z) \\
& =\frac{1}{s} \sum_{x \in \mathcal{X}} \mathbb{E}_{X^{n}}\left[T_{X^{n}}(x)\right] \log \sum_{z} P^{1+s}(z \mid x) Q^{-s}(z) \\
& =\frac{1}{s} \sum_{x \in \mathcal{X}} P_{X_{J}}^{(n)}(x) \log \sum_{z} P^{1+s}(z \mid x) Q^{-s}(z)
\end{aligned}
$$

Hence

$$
R_{0} \geq \limsup _{n \rightarrow \infty} \frac{1}{s} \sum_{x \in \mathcal{X}} P_{X_{J}}^{(n)}(x) \log \sum_{z} P^{1+s}(z \mid x) Q^{-s}(z) .
$$

Consider the blocklengths $\left\{n_{k}\right\}_{k=1}^{\infty}$. Since $P_{X_{J}}^{\left(n_{k}\right)}$ converges to $\widetilde{P}_{X},(421)$ and (437) respectively imply

$$
R_{0}+R_{1} \leq I_{\widetilde{P}}(X ; Y),
$$

and

$$
R_{0} \geq \frac{1}{s} \sum_{x \in \mathcal{X}} \widetilde{P}_{X}(x) \log \sum_{z} P^{1+s}(z \mid x) Q^{-s}(z) .
$$

Therefore, $\widetilde{P}_{X}$ is the desired distribution $P_{X}$ in (75). The proof for $s \in(0, \infty]$ case is complete.

Next we consider the case of $s \in(-1,0]$. This case can be proved by following steps similar to the proof of the traditional channel resolvability problem (or distributed channel synthesis problem) [9]. Observe

$$
\begin{aligned}
R_{0} & \geq \frac{1}{n} I\left(M_{0} ; Z^{n} \mid M_{1}\right) \\
& =\frac{1}{n} I\left(X^{n} ; Z^{n} \mid M_{1}\right) \\
& =\frac{1}{n} I\left(X^{n} ; Z^{n}\right)-\frac{1}{n} I\left(M_{1} ; Z^{n}\right) \\
& =\frac{1}{n} I\left(X^{n} ; Z^{n}\right)-\delta_{n} \\
& =\frac{1}{n} H\left(Z^{n}\right)-\frac{1}{n} H\left(Z^{n} \mid X^{n}\right)-\delta_{n} \\
& =H_{Q}(Z)-H\left(Z_{J} \mid X_{J}\right)-\delta_{n}+\delta_{n}^{\prime},
\end{aligned}
$$

where (443) and (445) follow from the facts $\left|P_{M_{1} Z^{n}}-P_{M_{1}} Q_{Z}^{n}\right| \rightarrow 0$ and $\left|P_{Z^{n}}-Q_{Z}^{n}\right| \rightarrow 0$, respectively.

Furthermore, since there exist some sequence $\left\{n_{k}\right\}_{k=1}^{\infty}$ such that $P_{X}^{\left(n_{k}\right)}$ converges to some distribution $\widetilde{P}_{X}$ such that $\widetilde{P}_{X} \in$ $\mathcal{P}\left(P_{Z \mid X}, Q_{Z}\right)$, we have

$$
\begin{aligned}
R_{0} & \geq H_{Q}(Z)-H\left(Z_{J} \mid X_{J}\right) \\
& =I_{\widetilde{P}}(X ; Z) .
\end{aligned}
$$

On the other hand,

$$
R_{0}+R_{1} \leq I_{\widetilde{P}}(X ; Y) .
$$

Combining (447) and (448) gives the desired result.

\section{APPENDIX I \\ PROOF OF THEOREM 6}

We first prove (78).

Achievability: We only consider the case $s \in(0,1]$. The achievability result for $s \in(-1,0]$ can be obtained from the result for the case $s \in(0,1]$ by letting $s \downarrow 0$.

We use a similar random code as the one given in Lemma 1. That is, we set $\mathcal{C}_{n}=\left\{W^{n}(m)\right\}_{m \in \mathcal{M}}$ with $W^{n}(m) \sim$ $P_{W^{n}}, m \in \mathcal{M}$, and set the encoder as $f_{\mathcal{C}_{n}}(m)=W^{n}(m)$. We insert a random mapping (virtual channel) between the encoder $f_{\mathcal{C}_{n}}(m)$ and the channel, which is denoted as $P_{X^{n} \mid W^{n}}$. For this cascaded code, we set the distributions $P_{W^{n}}\left(w^{n}\right) \propto \widetilde{P}_{W}^{n}\left(w^{n}\right) 1\left\{w^{n} \in \mathcal{T}_{\epsilon^{\prime}}^{n}\right\}$ and $P_{X^{n}} \mid W^{n}\left(x^{n} \mid w^{n}\right) \propto$ $\widetilde{P}_{X \mid W}^{n}\left(x^{n} \mid w^{n}\right) 1\left\{\left(w^{n}, x^{n}\right) \in \mathcal{T}_{\epsilon}^{n}\right\}$ for some $\widetilde{P}_{W X}$ such that $\widetilde{P}_{X} \in \mathcal{P}\left(P_{Z \mid X}, Q_{Z}\right)$, where $\epsilon^{\prime}<\epsilon$, and $\mathcal{T}_{\epsilon^{\prime}}^{n}, \mathcal{T}_{\epsilon}^{n}$, and $\mathcal{T}_{\epsilon}^{n}\left(w^{n}\right)$ respectively denote the typical set respect to $\widetilde{P}_{W}$, as well as the jointly typical set and conditional typical set respect to $\widetilde{P}_{W X}$.

Then by the method of types, we obtain (449)(455), where $\quad\left[\left(V_{X \mid W Z} \quad \circ \quad V_{Z \mid W}\right) T_{W}\right](x, w) \quad:=$ $\sum_{z} V_{X \mid W Z}(x \mid w, z) V_{Z \mid W}(z \mid x) T_{W}(w), \quad$ (452) follows since, by the law of large numbers, $\widetilde{P}_{W}^{n}\left(\mathcal{T}_{\epsilon^{\prime}}^{n}\right) \rightarrow 1$ and $\widetilde{P}_{X \mid W}^{n}\left(\mathcal{T}_{\epsilon}^{n}\left(w^{n}\right) \mid w^{n}\right) \rightarrow 1$ uniformly for all $w^{n} \in \mathcal{T}_{\epsilon^{\prime}}^{n}\left(\widetilde{P}_{W}\right)$ (this can be shown by following steps similar to the proof of conditional typicality lemma in [55], and hence the proof is omitted here), (453) follows from the fact that the number of $n$-types $T_{Y} V_{X \mid Y}$ is polynomial in $n$, and in (455) the arguments of maximization are replaced by $T_{W X}, V_{Z \mid W X}$ (this is feasible since both $\left(T_{W}, V_{Z \mid W}, V_{X \mid W Z}\right)$ in (454) and $\left(T_{W X}, V_{Z \mid W X}\right)$ in (455) run through all the types of sequences in $\left.\mathcal{X}^{n} \times \mathcal{Y}^{n} \times \mathcal{Z}^{n}\right)$.

Observe that in (455) $T_{W X}$ is restricted to being close to $\widetilde{P}_{W X}$ but there is no restriction on $V_{Z \mid W X}$. Actually Lemma 4 implies that as $n \rightarrow \infty$ and $\epsilon, \epsilon^{\prime} \rightarrow 0$, (455) asymptotically equals

$$
\begin{aligned}
& \underset{\widetilde{P}_{Z \mid W X}}{\max }\left\{-\frac{1+s}{s} \sum_{w, x, z} \widetilde{P}(w, x, z) \log \frac{\widetilde{P}(z \mid w, x)}{P(z \mid x)}\right. \\
& \left.+\sum_{w, z} \widetilde{P}(w, z) \log \frac{\widetilde{P}(z \mid w)}{Q(z)}\right\}
\end{aligned}
$$

in the sense that the difference between (455) and (456) vanishes as $n \rightarrow \infty$. That is, we can replace the (conditional) types with their corresponding (conditional) distributions. Hence $\frac{1}{n} D_{1+s}\left(P_{W^{n}} Z^{n} \| P_{W^{n}} \times Q_{Z}^{n}\right) \rightarrow$ (456) as $n \rightarrow \infty$. Comparing (456) to the definition of $\widetilde{R}_{1+s}^{\prime}\left(\widetilde{P}_{W \mid X} \widetilde{P}_{X}, P_{Z \mid X}, Q_{Z}\right)$ in (80), we can find that they are equal for the case of $s \in(0,1]$. Hence

$$
\begin{aligned}
& \lim _{n \rightarrow \infty} \frac{1}{n} D_{1+s}\left(P_{W^{n}} Z^{n} \| P_{W^{n}} \times Q_{Z}^{n}\right) \\
& =\widetilde{R}_{1+s}^{\prime}\left(\widetilde{P}_{W \mid X} \widetilde{P}_{X}, P_{Z \mid X}, Q_{Z}\right) .
\end{aligned}
$$




$$
\begin{aligned}
& \frac{1}{n} D_{1+s}\left(P_{W^{n}} Z^{n} \| P_{W^{n}} \times Q_{Z}^{n}\right) \\
& =\frac{1}{n s} \log \sum_{w^{n}, z^{n}} P\left(w^{n}\right) P^{1+s}\left(z^{n} \mid w^{n}\right) Q^{-s}\left(z^{n}\right) \\
& =\frac{1}{n s} \log \sum_{w^{n}, z^{n}} P\left(w^{n}\right)\left(\sum_{x^{n}} P\left(x^{n} \mid w^{n}\right) P\left(z^{n} \mid x^{n}\right)\right)^{1+s} Q^{-s}\left(z^{n}\right) \\
& =\frac{1}{n s} \log \sum_{T_{W}} \sum_{w^{n} \in \mathcal{T}_{T_{W}}} \sum_{V_{Z \mid W}} \sum_{z^{n} \in \mathcal{T}_{V_{Z \mid W}}\left(w^{n}\right)} \frac{\widetilde{P}\left(w^{n}\right) 1\left\{w^{n} \in \mathcal{T}_{\epsilon^{\prime}}^{n}\right\}}{\widetilde{P}_{W}^{n}\left(\mathcal{T}_{\epsilon^{\prime}}^{n}\right)} \\
& \times\left(\sum_{V_{X \mid W Z}} \sum_{x^{n} \in \mathcal{T}_{V_{X \mid W Z}}\left(w^{n}, z^{n}\right)} \frac{\widetilde{P}\left(x^{n} \mid w^{n}\right) 1\left\{\left(w^{n}, x^{n}\right) \in \mathcal{T}_{\epsilon}^{n}\right\}}{\widetilde{P}_{X \mid W}^{n}\left(\mathcal{T}_{\epsilon}^{n}\left(w^{n}\right) \mid w^{n}\right)} \mathrm{e}^{n \sum_{x, z} T(x, z) \log P(z \mid x)}\right)^{1+s} \mathrm{e}^{-n s \sum_{z} T(z) \log Q(z)} \\
& =\delta_{n}+\frac{1}{n s} \log \sum_{T_{W}} \sum_{w^{n} \in \mathcal{T}_{T_{W}}} \sum_{V_{Z \mid W}} \sum_{z^{n} \in \mathcal{T}_{V_{Z} \mid W}\left(w^{n}\right)} \widetilde{P}\left(w^{n}\right) 1\left\{w^{n} \in \mathcal{T}_{\epsilon^{\prime}}^{n}\right\} \\
& \times\left(\sum_{V_{X \mid W Z}} \sum_{x^{n} \in \mathcal{T}_{V_{X \mid W Z}}\left(w^{n}, z^{n}\right)} \widetilde{P}\left(x^{n} \mid w^{n}\right) 1\left\{\left(w^{n}, x^{n}\right) \in \mathcal{T}_{\epsilon}^{n}\right\} \mathrm{e}^{n \sum_{x, z} T(x, z) \log P(z \mid x)}\right)^{1+s} \mathrm{e}^{-n s \sum_{z} T(z) \log Q(z)} \\
& \leq \delta_{n}+\delta_{n}^{\prime}+\frac{1}{n s} \log \max _{T_{W}:\left|T_{W}-\widetilde{P}_{W}\right| \leq \epsilon^{\prime} V_{Z \mid W}} \sum_{w^{n} \in \mathcal{T}_{T_{W}}} \sum_{z^{n} \in \mathcal{T}_{V_{Z \mid W}}\left(w^{n}\right)} \widetilde{P}\left(w^{n}\right) \\
& \times\left(\max _{\left|\left(V_{X \mid W Z} \circ V_{Z \mid W}\right) T_{W}-\widetilde{P}_{W X}\right| \leq \epsilon} \sum_{x^{n} \in \mathcal{T}_{V_{X \mid W Z}}\left(w^{n}, z^{n}\right)} \widetilde{P}\left(x^{n} \mid w^{n}\right) \mathrm{e}^{n \sum_{x, z} T(x, z) \log P(z \mid x)}\right)^{1+s} \mathrm{e}^{-n s \sum_{z} T(z) \log Q(z)} \\
& =\max _{T_{W}, V_{Z \mid W}, V_{X \mid W Z}:\left|T_{W}-\widetilde{P}_{W}\right| \leq \epsilon^{\prime},} \frac{1}{s}\left(H\left(V_{Z \mid W} \times T_{W}\right)+\sum_{w} T(w) \log \widetilde{P}(w)\right) \\
& \left|\left(V_{X \mid W Z} \circ V_{Z \mid W}\right) T_{W}-\widetilde{P}_{W X}\right| \leq \epsilon \\
& +\frac{1+s}{s}\left(H\left(V_{X \mid W Z} \mid T_{W} V_{Z \mid W}\right)+\sum_{w, x} T(w, x) \log \widetilde{P}(x \mid w)+\sum_{x, z} T(x, z) \log P(z \mid x)\right) \\
& -\sum_{z} T(z) \log Q(z)+\delta_{n}+\delta_{n}^{\prime}+\delta_{n}^{\prime \prime} \\
& =\max _{T_{W X}, V_{Z \mid W X}:\left|T_{W}-\widetilde{P}_{W}\right| \leq \epsilon^{\prime},}\left\{\frac{1+s}{s} \sum_{w, x, z} T(w, x, z) \log \frac{\widetilde{P}(w, x) P(z \mid x)}{T(w, x, z)}+\sum_{w, z} T(w, z) \log \frac{T(w, z)}{\widetilde{P}(w) Q(z)}\right\} \\
& \left|T_{W X}-\widetilde{P}_{W X}\right| \leq \epsilon \\
& +\delta_{n}+\delta_{n}^{\prime}+\delta_{n}^{\prime \prime} \text {, }
\end{aligned}
$$

Furthermore, observe

$$
\begin{aligned}
& P_{X^{n}}\left(x^{n}\right) \\
& =\sum_{w^{n}} \frac{\widetilde{P}\left(w^{n}\right) 1\left\{w^{n} \in \mathcal{T}_{\epsilon^{\prime}}^{n}\right\}}{\widetilde{P}_{W}^{n}\left(\mathcal{T}_{\epsilon^{\prime}}^{n}\right)} \frac{\widetilde{P}\left(x^{n} \mid w^{n}\right) 1\left\{\left(w^{n}, x^{n}\right) \in \mathcal{T}_{\epsilon}^{n}\right\}}{\widetilde{P}_{X \mid W}^{n}\left(\mathcal{T}_{\epsilon}^{n}\left(w^{n}\right) \mid w^{n}\right)} \\
& \leq \sum_{w^{n}} \frac{\widetilde{P}\left(w^{n}\right) 1\left\{w^{n} \in \mathcal{T}_{\epsilon^{\prime}}^{n}\right\} \widetilde{P}\left(x^{n} \mid w^{n}\right) 1\left\{\left(w^{n}, x^{n}\right) \in \mathcal{T}_{\epsilon}^{n}\right\}}{1-\delta_{n}} \\
& \leq \frac{\widetilde{P}\left(x^{n}\right) 1\left\{x^{n} \in \mathcal{T}_{\epsilon}^{n}\right\}}{1-\delta_{n}},
\end{aligned}
$$

where (459) follows since as $n \rightarrow \infty, \widetilde{P}_{W}^{n}\left(\mathcal{T}_{\epsilon^{\prime}}^{n}\right)$ converges to 1 and $\widetilde{P}_{X \mid W}^{n}\left(\mathcal{T}_{\epsilon}^{n}\left(w^{n}\right) \mid w^{n}\right)$ uniformly converges to 1 for all $w^{n} \in \mathcal{T}_{\epsilon^{\prime}}^{n}$. Therefore,

$$
\begin{aligned}
& D_{1+s}\left(P_{X^{n}} \| \widetilde{P}_{X^{n}}\right) \\
& \leq \frac{1}{s} \log \sum_{x^{n}}\left(\frac{\widetilde{P}\left(x^{n}\right) 1\left\{x^{n} \in \mathcal{T}_{\epsilon}^{n}\right\}}{1-\delta_{n}}\right)^{1+s} \widetilde{P}^{-s}\left(x^{n}\right) \\
& =\frac{1}{s} \log \frac{\widetilde{P}_{X}^{n}\left(\mathcal{T}_{\epsilon}^{n}\right)}{\left(1-\delta_{n}\right)^{1+s}} \\
& \rightarrow 0,
\end{aligned}
$$

where (463) follows since $\widetilde{P}_{X}^{n}\left(\mathcal{T}_{\epsilon}^{n}\right)$ converges to 1 as $n \rightarrow \infty$. Since $P_{Z^{n}}$ and $Q_{Z}^{n}$ are respectively the distributions of the channel output induced by the input $P_{X^{n}}$ and $\widetilde{P}_{X}^{n}$, by the data processing inequality [35], we have

$$
D_{1+s}\left(P_{Z^{n}} \| Q_{Z}^{n}\right) \leq D_{1+s}\left(P_{X^{n}} \| \widetilde{P}_{X}^{n}\right) .
$$

Hence $D_{1+s}\left(P_{Z^{n}} \| Q_{Z}^{n}\right) \rightarrow 0$ as well. 
Finally, by Lemma 1, we obtain

$$
\begin{aligned}
& \mathrm{e}^{s D_{1+s}\left(P_{M_{1} Z^{n}} \| P_{M_{1}} Q_{Z}^{n}\right)} \\
& \leq \mathrm{e}^{s D_{1+s}\left(P_{W^{n}} Z^{n} \| P_{W^{n}} Q_{Z}^{n}\right)-n s R_{0}}+\mathrm{e}^{s D_{1+s}\left(P_{Z^{n}} \| Q_{Z}^{n}\right)} \\
& \quad \rightarrow 1
\end{aligned}
$$

where (466) holds for $s \in(0,1]$ if

$$
R_{0}>\widetilde{R}_{1+s}^{\prime}\left(\widetilde{P}_{W \mid X} \widetilde{P}_{X}, P_{Z \mid X}, Q_{Z}\right)
$$

by (456) with a small enough $\epsilon>0$. Hence the secrecy constraint is satisfied.

Moreover, using standard joint typicality decoding, we have that error constraint

$$
\mathbb{P}\left(\left(M_{0}, M_{1}\right) \neq\left(\widehat{M}_{0}, \widehat{M}_{1}\right)\right) \rightarrow 0
$$

is satisfied as well if $R_{0}+R_{1} \leq I_{\widetilde{P}}(W ; Y)$. The proof of the achievability part for $s \in[-1,1]$ is complete. For $s=\infty$, the achievability part can be proven by similar steps to those in Appendix 5.

Converse: Set $W=\left(M_{0}, M_{1}\right)$. By the data processing inequality,

$$
R_{0}+R_{1} \leq \frac{1}{n} I\left(W ; Y^{n}\right) \leq I\left(W ; Y_{J}\right),
$$

where $J \sim$ Unif $[1: n]$ denotes a time index variable, independent of $\left(W, Y^{n}\right)$. It is easy to verify that

$$
\begin{aligned}
& P_{W X_{J} Y_{J}}(w, x, y) \\
& =P_{W}(w) \frac{1}{n} \sum_{j=1}^{n} \mathbb{P}\left\{\left(X_{j}, Y_{j}\right)=(x, y) \mid W=w\right\} \\
& =P_{W}(w) \mathbb{E}_{X^{n} Y^{n} \mid W=w}\left[T_{X^{n} Y^{n}}(x, y)\right],
\end{aligned}
$$

and

$$
\begin{aligned}
& P_{W X_{J} Y_{J}}(w, x, y) \\
& =P_{W X_{J}}(w, x) P(y \mid x) \\
& =P_{W}(w) \mathbb{E}_{X^{n} \mid W=w}\left[T_{X^{n}}(x)\right] P(y \mid x),
\end{aligned}
$$

where (473) is obtained similarly to (470)-(471).

We first consider the case $s \in(0, \infty]$. Observe $M_{1}$ is independent of $M_{0}$. Hence if we consider $M_{1}$ as $\mathcal{C}$ and $M_{0}$ as $M$, then the wiretap channel problem turns into the channel resolvability problem. By Lemma 2, we obtain

$$
\begin{aligned}
& D_{1+s}\left(P_{M_{1} Z^{n}} \| P_{M_{1}} Q_{Z}^{n}\right) \\
& \geq \max \left\{D_{1+s}\left(P_{M_{0} M_{1} Z^{n}} \| P_{M_{0} M_{1}} \times Q_{Z}^{n}\right)-n R_{0}\right. \\
& \left.\quad D_{1+s}\left(P_{Z^{n}} \| Q_{Z}^{n}\right)\right\} \\
& =\max \left\{D_{1+s}\left(P_{W Z^{n}} \| P_{W} \times Q_{Z}^{n}\right)-n R_{0}\right. \\
& \left.\quad D_{1+s}\left(P_{Z^{n}} \| Q_{Z}^{n}\right)\right\}
\end{aligned}
$$

Define $\widetilde{P}_{Z \mid W X}$ as the maximizing distribution of

$$
\begin{aligned}
& \max _{\widetilde{P}_{Z \mid W X} \in \mathcal{P}(\mathcal{Z} \mid \mathcal{W} \times \mathcal{X})}\left\{-\frac{1+s}{s} \sum_{w, x, z} P(w) P_{X_{J} \mid W}(x \mid w)\right. \\
& \times \widetilde{P}(z \mid w, x) \log \frac{\widetilde{P}(z \mid w, x)}{P(z \mid x)} \\
& +\sum_{w, x, z} P(w) P_{X_{J} \mid W}(x \mid w) \widetilde{P}(z \mid w, x) \\
& \left.\quad \times \log \frac{\sum_{x} P_{X_{J} \mid W}(x \mid w) \widetilde{P}(z \mid w, x)}{Q(z)}\right\}
\end{aligned}
$$

where $P_{W X_{J} Z_{J}}$ is the distribution of $W, X_{J}, Z_{J}$ induced by the code. Note that $\widetilde{P}_{Z \mid W X}$ is determined by the code, the channel $P_{Z \mid X}$, and the target distribution $Q_{Z}$.

From Lemma 3 we know that for any $w \in \mathcal{W}$ and any $T_{X} \in$ $\mathcal{P}^{(n)}(\mathcal{X})$, we can find a conditional type $V_{Z \mid X}^{(w)} \in \mathcal{P}^{(n)}\left(\mathcal{X} \mid T_{X}\right)$ such that

$$
\left|T_{X} \widetilde{P}_{Z \mid X W}(\cdot \mid \cdot, w)-T_{X} V_{Z \mid X}^{(w)}\right| \leq \frac{|\mathcal{X}||\mathcal{Z}|}{2 n}=O\left(\frac{1}{n}\right) .
$$

Consider the first term of the maximization in (475), then we obtain (478)-(490), where (480) follows from Lemma 6, (481) and (485) follow since $x \mapsto x^{1+s}$ is a convex function for $s \geq 0$, (482) follows from Lemma 5 , in (483) $V_{Z \mid X}^{(w)}: \mathcal{W} \rightarrow$ $\mathcal{P}^{(n)}\left(\mathcal{Z} \mid T_{X}\right)$ is the conditional type above satisfying (477), ${ }^{12}$ (484) follows from (477) and [57, Lem. 8], (486) follows since the number of types in $\mathcal{P}^{(n)}(\mathcal{X})$ is polynomial in $n$, (487) follows since $x \mapsto \log x$ is a concave function, (488) follows since $P_{X^{n} \mid W}\left(\mathcal{T}_{T_{X}} \mid w\right)=\sum_{x^{n} \in \mathcal{T}_{T_{X}}} P_{X^{n} \mid W}\left(x^{n} \mid w\right)$ and $\mathcal{T}_{T_{X}} \subseteq \mathcal{X}^{n}$ runs through all the sequences in $\mathcal{X}^{n}$, (489) follows since $x \mapsto x \log x$ is a convex function, and (490) follows since $\mathbb{E}_{X^{n} \mid W=w}\left[T_{X^{n}}(x)\right]=P_{X_{J} \mid W}(x \mid w)$; see (473).

By the choice of $\widetilde{P}_{Z \mid W X}$, from (490) we have

$$
\lim _{n \rightarrow \infty} \frac{1}{n} D_{1+s}\left(P_{W Z^{n}} \| P_{W} \times Q_{Z}^{n}\right) \geq(476) .
$$

Furthermore, it is easy to verify

$$
\lim _{n \rightarrow \infty}\left|P_{X_{J}}^{(n)} \circ P_{Z \mid X}-Q_{Z}\right|=0,
$$

since $D_{1+s}\left(P_{Z^{n}} \| Q_{Z^{n}}\right) \rightarrow 0$ (see (475)).

Since $\mathcal{P}(\mathcal{X})$ is compact, for each $w$, there must exist some sequence of increasing integers $\left\{n_{k}\right\}_{k=1}^{\infty}$ such that $P_{X_{J} \mid W=w}^{\left(n_{k}\right)}$ converges to some distribution $\widetilde{P}_{X \mid W=w}$. By (492), $\mathbb{E}_{W}\left[\widetilde{P}_{X \mid W}(\cdot \mid W)\right] \in \mathcal{P}\left(P_{Z \mid X}, Q_{Z}\right)$ holds. Moreover, (469) and (491) respectively imply

$$
R_{0}+R_{1} \leq I_{\widetilde{P}}(W ; Y),
$$

and

$$
\begin{aligned}
R_{0} \geq \max _{\widetilde{P}_{Z \mid W X}}\{- & \frac{1+s}{s} \sum_{w, x, z} \widetilde{P}(w, x, z) \log \frac{\widetilde{P}(z \mid w, x)}{P(z \mid x)} \\
& \left.+\sum_{w, z} \widetilde{P}(w, z) \log \frac{\widetilde{P}(z \mid w)}{Q(z)}\right\}
\end{aligned}
$$

\footnotetext{
${ }^{12}$ Note that the choice of $V_{Z \mid X}^{(w)}$ and not necessarily an optimal one for the lower bound (483), since the optima should be independent of $w$. However, it is, in fact, optimal for the final lower bound (489).
} 


$$
\begin{aligned}
& \frac{1}{n} D_{1+s}\left(P_{W Z^{n}} \| P_{W} \times Q_{Z}^{n}\right) \\
& =\frac{1}{n s} \log \sum_{w \in \mathcal{W}} \sum_{T_{Z}} \sum_{z^{n} \in \mathcal{T}_{T_{Z}}} P(w) \mathrm{e}^{-n s \sum_{z} T(z) \log Q(z)}\left(\sum_{V_{X \mid Z}} \sum_{x^{n} \in \mathcal{T}_{V_{X \mid Z}}\left(z^{n}\right)} P\left(x^{n} \mid w\right) \mathrm{e}^{n \sum_{x, z} T(x, z) \log P(z \mid x)}\right)^{1+s} \\
& =\frac{1}{n s} \log \sum_{w \in \mathcal{W}} \sum_{T_{Z}} \sum_{z^{n} \in \mathcal{T}_{T_{Z}}} P(w) \mathrm{e}^{-n s \sum_{z} T(z) \log Q(z)}\left(\sum_{V_{X \mid Z}} P_{X^{n} \mid W}\left(\mathcal{T}_{V_{X \mid Z}}\left(z^{n}\right) \mid w\right) \mathrm{e}^{n \sum_{x, z} T(x, z) \log P(z \mid x)}\right)^{1+s} \\
& \geq \frac{1}{n s} \log \sum_{w \in \mathcal{W}} \sum_{T_{Z}} \sum_{z^{n} \in \mathcal{T}_{T_{Z}}} P(w) \sum_{V_{X \mid Z}} P_{X^{n} \mid W}^{1+s}\left(\mathcal{T}_{V_{X \mid Z}}\left(z^{n}\right) \mid w\right) \mathrm{e}^{n(1+s) \sum_{x, z} T(x, z) \log P(z \mid x)-n s \sum_{z} T(z) \log Q(z)} \\
& \geq \frac{1}{n s} \log \sum_{w, T_{Z}, V_{X \mid Z}}\left|\mathcal{T}_{T_{Z}}\right| P(w)\left(\sum_{z^{n} \in \mathcal{T}_{T_{Z}}} \frac{1}{\left|\mathcal{T}_{T_{Z} \mid}\right|} P_{X^{n} \mid W}\left(\mathcal{T}_{V_{X \mid Z}}\left(z^{n}\right) \mid w\right)\right)^{1+s} \mathrm{e}^{n(1+s) \sum_{x, z} T(x, z) \log P(z \mid x)-n s \sum_{z} T(z) \log Q(z)} \\
& =\delta_{n}+\frac{1}{n s} \log \sum_{w, T_{Z}, V_{X \mid Z}} P(w) P_{X^{n} \mid W}^{1+s}\left(\mathcal{T}_{T_{X}} \mid w\right) \mathrm{e}^{-n s H\left(T_{Z}\right)+n(1+s) H\left(V_{Z \mid X} \mid T_{X}\right)} \mathrm{e}^{n(1+s) \sum_{x, z} T(x, z) \log P(z \mid x)-n s \sum_{z} T(z) \log Q(z)} \\
& \geq \delta_{n}+\frac{1}{n s} \log \sum_{w, T_{X}} P(w) P_{X^{n} \mid W}^{1+s}\left(\mathcal{T}_{T_{X}} \mid w\right) \mathrm{e}^{-n s H\left(V_{Z \mid X}^{(w)} \circ T_{X}\right)+n(1+s)\left(H\left(V_{Z \mid X}^{(w)} \mid T_{X}\right)+\sum_{x, z} T(x) V_{Z \mid X}^{(w)}(z \mid x) \log P(z \mid x)\right)} \\
& \times \mathrm{e}^{-n s \sum_{z}\left[V_{Z \mid X}^{(w)} \circ T_{X}\right](z) \log Q(z)} \\
& =\delta_{n}+\frac{1}{n s} \log \sum_{w, T_{X}} P(w) P_{X^{n} \mid W}^{1+s}\left(\mathcal{T}_{T_{X}} \mid w\right) \mathrm{e}^{-n s H\left(\widetilde{P}_{Z \mid W X} \circ T_{X}\right)+n(1+s)\left(H\left(\widetilde{P}_{Z \mid W X} \mid T_{X}\right)+\sum_{x, z} T(x) \widetilde{P}_{Z \mid W X}(z \mid x) \log P(z \mid x)\right)} \\
& \times \mathrm{e}^{-n s \sum_{z}\left[\widetilde{P}_{Z \mid W X} \circ T_{X}\right](z) \log Q(z)+n \cdot \delta_{n}^{\prime}} \\
& \geq \delta_{n}+\delta_{n}^{\prime}+\frac{1}{n s} \log \left|\mathcal{P}^{(n)}(\mathcal{X})\right|\left(\sum_{w, T_{X}} \frac{1}{\left|\mathcal{P}^{(n)}(\mathcal{X})\right|} P(w) P_{X^{n} \mid W}\left(\mathcal{T}_{T_{X}} \mid w\right)\right. \\
& \left.\times \mathrm{e}^{-n \sum_{x, z} T(x) \widetilde{P}(z \mid w, x) \log \frac{\tilde{P}(z \mid w, x)}{P(z \mid x)}+\frac{n s}{1+s} \sum_{x, z} T(x) \widetilde{P}(z \mid w, x) \log \frac{\sum_{x} T(x) \widetilde{P}(z \mid w, x)}{Q(z)}}\right)^{1+s} \\
& \geq \delta_{n}+\delta_{n}^{\prime}+\delta_{n}^{\prime \prime}+\frac{1+s}{n s} \log \sum_{w, T_{X}} P(w) P_{X^{n} \mid W}\left(\mathcal{T}_{T_{X}} \mid w\right) \\
& \times \mathrm{e}^{-n \sum_{x, z} T(x) \widetilde{P}(z \mid w, x) \log \frac{\widetilde{P}(z \mid w, x)}{P(z \mid x)}+\frac{n s}{1+s} \sum_{x, z} T(x) \widetilde{P}(z \mid w, x) \log \frac{\sum_{x} T(x) \widetilde{P}(z \mid w, x)}{Q(z)}} \\
& \geq \delta_{n}+\delta_{n}^{\prime}+\delta_{n}^{\prime \prime}+\sum_{w, T_{X}} P(w) P_{X^{n} \mid W}\left(\mathcal{T}_{T_{X}} \mid w\right)\left(-\frac{1+s}{s} \sum_{x, z} T_{X}(x) \widetilde{P}(z \mid w, x) \log \frac{\widetilde{P}(z \mid w, x)}{P(z \mid x)}\right. \\
& \left.+\sum_{x, z} T_{X}(x) \widetilde{P}(z \mid w, x) \log \frac{\sum_{x} T_{X}(x) \widetilde{P}(z \mid w, x)}{Q(z)}\right) \\
& =\delta_{n}+\delta_{n}^{\prime}+\delta_{n}^{\prime \prime}+\sum_{w \in \mathcal{W}} P(w)\left(-\frac{1+s}{s} \sum_{x, z} \mathbb{E}_{X^{n} \mid W=w}\left[T_{X^{n}}(x)\right] \widetilde{P}(z \mid w, x) \log \frac{\widetilde{P}(z \mid w, x)}{P(z \mid x)}\right. \\
& \left.+\sum_{x, z} \mathbb{E}_{X^{n} \mid W=w}\left[T_{X^{n}}(x) \widetilde{P}(z \mid w, x) \log \frac{\sum_{x} T_{X^{n}}(x) \widetilde{P}(z \mid w, x)}{Q(z)}\right]\right) \\
& \geq \delta_{n}+\delta_{n}^{\prime}+\delta_{n}^{\prime \prime}+\sum_{w \in \mathcal{W}} P(w)\left(-\frac{1+s}{s} \sum_{x, z} \mathbb{E}_{X^{n} \mid W=w}\left[T_{X^{n}}(x)\right] \widetilde{P}(z \mid w, x) \log \frac{\widetilde{P}(z \mid w, x)}{P(z \mid x)}\right. \\
& \left.+\sum_{x, z} \mathbb{E}_{X^{n} \mid W=w}\left[T_{X^{n}}(x)\right] \widetilde{P}(z \mid w, x) \log \frac{\sum_{x} \mathbb{E}_{X^{n} \mid W=w}\left[T_{X^{n}}(x)\right] \widetilde{P}(z \mid w, x)}{Q(z)}\right)
\end{aligned}
$$




$$
\begin{aligned}
=\delta_{n} & +\delta_{n}^{\prime}+\delta_{n}^{\prime \prime}+\sum_{w \in \mathcal{W}} P(w)\left(-\frac{1+s}{s} \sum_{x, z} P_{X_{J} \mid W}(x \mid w) \widetilde{P}(z \mid w, x) \log \frac{\widetilde{P}(z \mid w, x)}{P(z \mid x)}\right. \\
& \left.+\sum_{x, z} P_{X_{J} \mid W}(x \mid w) \widetilde{P}(z \mid w, x) \log \frac{\sum_{x} P_{X_{J} \mid W}(x \mid w) \widetilde{P}(z \mid w, x)}{Q(z)}\right) .
\end{aligned}
$$

Observe that the RHS of (494) is just $\widetilde{R}_{1+s}^{\prime}\left(\widetilde{P}_{W \mid X} \widetilde{P}_{X}, P_{Z \mid X}, Q_{Z}\right)$ with $s \in(0, \infty]$. Hence $R_{0} \geq \widetilde{R}_{1+s}^{\prime}\left(\widetilde{P}_{W \mid X} \widetilde{P}_{X}, P_{Z \mid X}, Q_{Z}\right)$.

Therefore, $P_{W} \widetilde{P}_{X \mid W}$ is the desired distribution $\widetilde{P}_{W X}$ in (78). The proof for the case $s \in(0, \infty]$ is complete.

Next we consider the case $s \in(-1,0]$. This case can be proved by following steps similar to the proof of traditional channel resolvability problem [2] or the distributed channel synthesis problem [9]. Observe

$$
\begin{aligned}
R_{0} & \geq \frac{1}{n} I\left(M_{0} ; Z^{n} \mid M_{1}\right) \\
& =\frac{1}{n} I\left(M_{0} M_{1} ; Z^{n} \mid M_{1}\right) \\
& =\frac{1}{n} I\left(M_{0} M_{1} ; Z^{n}\right)-\frac{1}{n} I\left(M_{1} ; Z^{n}\right) \\
& =\frac{1}{n} H\left(Z^{n}\right)-\frac{1}{n} H\left(Z^{n} \mid M_{0} M_{1}\right)-\delta_{n} \\
& =H_{Q}(Z)-H\left(Z_{J} \mid W\right)-\delta_{n}+\delta_{n}^{\prime},
\end{aligned}
$$

where (498) and (499) follow from the facts $\left|P_{M_{1} Z^{n}}-P_{M_{1}} Q_{Z}^{n}\right| \rightarrow 0$ and $\left|P_{Z^{n}}-Q_{Z}^{n}\right| \rightarrow 0$ respectively.

Furthermore, for each $w$, there exists some increasing sequence of integers $\left\{n_{k}\right\}_{k=1}^{\infty}$ such that $P_{X_{J} \mid W=w}^{\left(n_{k}\right)}$ converges to some distribution $\widetilde{P}_{X \mid W=w}$ that satisfies $\mathbb{E}_{W}\left[\widetilde{P}_{X \mid W}(\cdot \mid W)\right] \in$ $\mathcal{P}\left(P_{Z \mid X}, Q_{Z}\right)$. Hence letting $n=n_{k}$ and $k \rightarrow \infty$ in (499), we get

$$
R_{0} \geq H_{\widetilde{P}}(Z)-H_{\widetilde{P}}\left(Z_{J} \mid W\right)=I_{\widetilde{P}}(W ; Z) .
$$

On the other hand,

$$
R_{0}+R_{1} \leq I_{\widetilde{P}}(W ; Y) .
$$

Combining (500) and (501) gives the converse part. Therefore, the proof of (78) is complete.

Next we prove (79). By adding an artificial non-secret message $M_{0}^{\prime}$ (with rate $R_{0}^{\prime}$ ) in the achievability scheme above, we have the following achievable region.

$$
\underset{\widetilde{P}_{X} \in \mathcal{P}\left(P_{Z \mid X}, Q_{Z}\right)}{\bigcup}\left\{\begin{array}{l}
\left(R_{0}, R_{1}\right): R_{0}^{\prime} \geq 0 \\
R_{0}^{\prime}+R_{0}+R_{1} \leq I_{\widetilde{P}}(W ; Y), \\
R_{0}^{\prime}+R_{0} \geq \\
\widetilde{R}_{1+s}^{\prime}\left(\widetilde{P}_{W \mid X} \widetilde{P}_{X}, P_{Z \mid X}, Q_{Z}\right)
\end{array}\right\} .
$$

Using Fourier-Motzkin Elimination (see [55, Appendix D]), we can show that the regions in (502) and (79) are the same. Hence (79) $\subseteq \mathcal{R}_{1+s}\left(Q_{Z}\right)$. On the other hand, comparing the RHSes of (78) and (79) yields that the RHS of (78) $\subseteq$ (79). In addition, $\mathcal{R}_{1+s}\left(Q_{Z}\right)=$ the RHS of (78). Hence $\mathcal{R}_{1+s}\left(Q_{Z}\right) \subseteq$ (79). Therefore, $\mathcal{R}_{1+s}\left(Q_{Z}\right)=$ (79).
Lastly, by standard cardinality bounding techniques [55, Appendix C], the alphabet size of $W$ can be limited to $|\mathcal{W}| \leq|\mathcal{X}|+1$.

\section{Acknowledgements}

The authors would like to thank Prof. Masahito Hayashi for pointing out the relevance of $[29$, Thm. 14] to the present work.

The authors are extremely grateful to the Associate Editor Prof. Matthieu Bloch and the two reviewers for their extensive, constructive and helpful feedback to improve the manuscript.

\section{REFERENCES}

[1] L. Yu and V. Y. F. Tan. Rényi resolvability and its applications to the wiretap channel. In International Conference on Information Theoretic Security, pages 208-233. Springer, 2017.

[2] T. Han and S. Verdú. Approximation theory of output statistics. IEEE Trans. on Inform. Theory, 39(3):752-772, 1993.

[3] M. Hayashi. General nonasymptotic and asymptotic formulas in channel resolvability and identification capacity and their application to the wiretap channel. IEEE Trans. on Inform. Theory, 52(4):1562-1575, 2006.

[4] M. Hayashi. Exponential decreasing rate of leaked information in universal random privacy amplification. IEEE Trans. on Inform. Theory, 57(6):3989-4001, 2011.

[5] J. Liu, P. Cuff, and S. Verdú. $E_{\gamma}$-resolvability. IEEE Trans. on Inform. Theory, 63(5):2629-2658, 2017.

[6] L. Yu and V. Y. F. Tan. Simulation of random variables under Rényi divergence measures of all orders. arXiv preprint 1805.12451, 2018.

[7] L. Yu and V. Y. F. Tan. Asymptotic coupling and its applications in information theory. IEEE Trans. on Inform. Theory, 65, 2019.

[8] A. Wyner. The common information of two dependent random variables. IEEE Trans. on Inform. Theory, 21(2):163-179, 1975.

[9] P. Cuff. Distributed channel synthesis. IEEE Trans. on Inform. Theory, 59(11):7071-7096, 2013.

[10] M. R. Bloch and J. N. Laneman. Strong secrecy from channel resolvability. IEEE Trans. on Inform. Theory, 59(12):8077-8098, 2013.

[11] T. S. Han, H. Endo, and M. Sasaki. Reliability and secrecy functions of the wiretap channel under cost constraint. IEEE Trans. on Inform. Theory, 60(11):6819-6843, 2014.

[12] M. B. Parizi, E. Telatar, and N. Merhav. Exact random coding secrecy exponents for the wiretap channel. IEEE Trans. on Inform. Theory, 63(1):509-531, 2017.

[13] J. Hou and G. Kramer. Effective secrecy: Reliability, confusion and stealth. In Information Theory (ISIT), 2014 IEEE International Symposium on, pages 601-605. IEEE, 2014.

[14] G. R. Kumar, C. T. Li, and A. El Gamal. Exact common information. In IEEE International Symposium on Information Theory (ISIT), pages 161-165. IEEE, 2014.

[15] C. T. Li and A. El Gamal. Distributed simulation of continuous random variables. IEEE Trans. on Inform. Theory, 63(10):6329-6343, 2017.

[16] L. Yu and V. Y. F. Tan. On exact and $\infty$-Rényi common informations. arXiv preprint 1810.00295, 2018.

[17] M. Iwamoto and J. Shikata. Information theoretic security for encryption based on conditional Rényi entropies. Lecture Notes in Computer Science (Information Theoretic Security), 8317:103-121, 2014.

[18] J. Shikata. Design and analysis of information-theoretically secure authentication codes with non-uniformly random keys. IACR Cryptology ePrint Archive, 2015:250, 2015. 
[19] S. Bai, A. Langlois, T. Lepoint, D. Stehlé, and R. Steinfeld. Improved security proofs in lattice-based cryptography: Using the Rényi divergence rather than the statistical distance. In Advances in CryptologyASIACRYPT 2015, pages 3-24, Berlin, Heidelberg, 2015. Springer Berlin Heidelberg.

[20] L. Yu and V. Y. F. Tan. Wyner's common information under Rényi divergence measures. IEEE Trans. on Inform. Theory, 64(5):3616-3632, 2018.

[21] L. Yu and V. Y. F. Tan. Corrections to "Wyner's common information under Rényi divergence measures". arXiv preprint arXiv:1810.02534, 2018.

[22] A. R. Barron. Entropy and the central limit theorem. The Annals of Probability, pages 336-342, 1986.

[23] S. G. Bobkov, G. P. Chistyakov, and F. Götze. Rényi divergence and the central limit theorem. arXiv preprint arXiv:1608.01805, 2016.

[24] S. Beigi and A. Gohari. Quantum achievability proof via collision relative entropy. IEEE Trans. on Inform. Theory, 60(12):7980-7986, 2014.

[25] Y. Dodis and Y. Yu. Overcoming weak expectations. In Theory of Cryptography, pages 1-22. Springer, 2013.

[26] M. Hayashi and V. Y. F. Tan. Equivocations, exponents, and secondorder coding rates under various Rényi information measures. IEEE Trans. on Inform. Theory, 63(2):975-1005, 2017.

[27] V. Y. F. Tan and M. Hayashi. Analysis of remaining uncertainties and exponents under various conditional Rényi entropies. IEEE Trans. on Inform. Theory, 64(5), 2018.

[28] R. A. Chou, B. N. Vellambi, M. R. Bloch, and J. Kliewer. Coding schemes for achieving strong secrecy at negligible cost. IEEE Trans. on Inform. Theory, 63(3):1858-1873, 2017.

[29] M. Hayashi and R. Matsumoto. Secure multiplex coding with dependent and non-uniform multiple messages. IEEE Trans. on Inform. Theory, 62(5):2355-2409, 2016

[30] I. Csiszár and J. Körner. Broadcast channels with confidential messages. IEEE Trans. on Inform. Theory, 24(3):339-348, 1978.

[31] I. Csiszár and J. Körner. Information Theory: Coding Theorems for Discrete Memoryless Systems. Cambridge University Press, 2011.

[32] B. N. Vellambi and J. Kliewer. Sufficient conditions for the equality of exact and Wyner common information. In Communication, Control, and Computing (Allerton), 2016 54th Annual Allerton Conference on, pages 370-377. IEEE, 2016.

[33] B. N. Vellambi and J. Kliewer. New results on the equality of exact and Wyner common information rates. In 2018 IEEE International Symposium on Information Theory (ISIT), pages 151-155. IEEE, 2018.

[34] L. Yu and V. Y. F. Tan. Exact channel synthesis. arXiv preprint arXiv:1810.13246, 2018.

[35] T. Van Erven and P. Harremos. Rényi divergence and Kullback-Leible divergence. IEEE Trans. on Inform. Theory, 60(7):3797-3820, 2014.

[36] S. Verdú. $\alpha$-mutual information. In Information Theory and Applications Workshop (ITA), pages 1-6, 2015.

[37] S. L. Fong and V. Y. F. Tan. Strong converse theorems for classes of multimessage multicast networks: A Rényi divergence approach. IEEE Trans. on Inform. Theory, 62(9):4953-4967, 2016.

[38] C. Cachin. Entropy measures and unconditional security in cryptography. PhD thesis, ETH Zurich, 1997.

[39] S. Fehr and S. Berens. On the conditional Rényi entropy. IEEE Trans. on Inform. Theory, 60(11):6801-6810, 2014.

[40] A. Wyner. The wire-tap channel. Bell Labs Technical Journal, 54(8):1355-1387, 1975.

[41] I. Csiszár and P. Narayan. Secrecy capacities for multiple terminals. IEEE Trans. on Inform. Theory, 50(12):3047-3061, 2004.

[42] S. Watanabe and Y. Oohama. The optimal use of rate-limited randomness in broadcast channels with confidential messages. IEEE Trans. on Inform. Theory, 61(2):983-995, 2015.

[43] J. Xu and B. Chen. Broadcast confidential and public messages. In Information Sciences and Systems, 2008. CISS 2008. 42nd Annual Conference on, pages 630-635. IEEE, 2008.

[44] D. Kobayashi, H. Yamamoto, and T. Ogawa. Secure multiplex coding attaining channel capacity in wiretap channels. IEEE Trans. on Inform. Theory, 59(12):8131-8143, 2013.

[45] A. Gohari and V. Anantharam. Generating dependent random variables over networks. In Information Theory Workshop (ITW), 2011 IEEE, pages 698-702, 2011.

[46] Z. Goldfeld, P. Cuff, and H. H. Permuter. Semantic-security capacity for wiretap channels of type II. IEEE Trans. on Inform. Theory, 62(7):38633879, 2016.

[47] P. Cuff, H. Permuter, and T. Cover. Coordination capacity. IEEE Trans. on Inform. Theory, 56(9):4181-4206, 2010.
[48] A. Dembo and O. Zeitouni. Large Deviations Techniques and Applications. Springer-Verlag, 2nd edition, 1998.

[49] R. G. Gallager. Information Theory and Reliable Communication, volume 2. Springer, 1968.

[50] V. Y. F. Tan, A. Anandkumar, L. Tong, and A. S. Willsky. A largedeviation analysis of the maximum-likelihood learning of Markov tree structures. IEEE Trans. on Inform. Theory, 57(3):1714-1735, 2011.

[51] M. Sion. On general minimax theorems. Pacific J. Math, 8(1):171-176, 1958.

[52] T. M. Cover and J. A. Thomas. Elements of Information Theory. WileyInterscience, 2nd edition, 2006

[53] S. Boucheron, G. Lugosi, and P. Massart. Concentration inequalities: A nonasymptotic theory of independence. Oxford university press, 2013.

[54] M. Mitzenmacher and E. Upfal. Probability and Computing: Randomized Algorithms and Probabilistic Analysis. Cambridge university press, 2005.

[55] A. El Gamal and Y.-H. Kim. Network Information Theory. Cambridge university press, 2011.

[56] M. Bloch and J. Barros. Physical-layer Security: From Information Theory to Security Engineering. Cambridge University Press, 2011.

[57] M. Yassaee, A. Gohari, and M. Aref. Channel simulation via interactive communications. IEEE Trans. on Inform. Theory, 61(6):2964-2982, 2015.

Lei Yu received the B.E. and Ph.D. degrees, both in electronic engineering, from University of Science and Technology of China (USTC) in 2010 and 2015, respectively. From 2015 to 2017, he was a postdoctoral researcher at the Department of Electronic Engineering and Information Science (EEIS), USTC. Currently, he is a research fellow at the Department of Electrical and Computer Engineering, National University of Singapore. His research interests include information theory, probability theory, and discrete mathematics.

Vincent Y. F. Tan (S'07-M'11-SM'15) was born in Singapore in 1981. He is currently a Dean's Chair Associate Professor in the Department of Electrical and Computer Engineering and the Department of Mathematics at the National University of Singapore (NUS). He received the B.A. and M.Eng. degrees in Electrical and Information Sciences from Cambridge University in 2005 and the Ph.D. degree in Electrical Engineering and Computer Science (EECS) from the Massachusetts Institute of Technology (MIT) in 2011. His research interests include information theory, machine learning, and statistical signal processing.

Dr. Tan received the MIT EECS Jin-Au Kong outstanding doctoral thesis prize in 2011, the NUS Young Investigator Award in 2014, the NUS Engineering Young Researcher Award in 2018, and the Singapore National Research Foundation (NRF) Fellowship (Class of 2018). He is also an IEEE Information Theory Society Distinguished Lecturer for 2018/9. He has authored a research monograph on "Asymptotic Estimates in Information Theory with Non-Vanishing Error Probabilities" in the Foundations and Trends in Communications and Information Theory Series (NOW Publishers). $\mathrm{He}$ is currently serving as an Associate Editor of the IEEE Transactions on Signal Processing. 